\title{
Estrogen-related receptor agonism reverses mitochondrial dysfunction and inflammation in the aging kidney
}

Xiaoxin X. Wang ${ }^{1}$, Komuraiah Myakala ${ }^{1}$, Andrew E. Libby ${ }^{1}$, Julia Panov ${ }^{2,3}$, Suman Ranjit ${ }^{1}$, Shogo Takahashi ${ }^{1,4}$, Bryce A. Jones ${ }^{5}$, Kanchan Bhasin ${ }^{1}$, Yue Qi ${ }^{6}$, Kristopher W. Krausz ${ }^{4}$, Patricia M. Zerfas ${ }^{7}$, Thomas J. Velenosi ${ }^{4}$, Daxesh P. Patel ${ }^{4}$, Parnaz Daneshpajouhnejad ${ }^{8}$, Avi Titievsky $^{2}$, Vadim Sharov $^{2}$, Boris Ostretsov ${ }^{2}$, Cyrielle Billon ${ }^{9}$, Arindam Chatterjee ${ }^{10}$, John K. Walker $^{10}$, Jeffrey B. Kopp ${ }^{11}$, Avi Z. Rosenberg ${ }^{8}$, Frank J. Gonzalez ${ }^{4}$, Udayan Guha ${ }^{6}$, Leonid Brodsky ${ }^{2}$, Thomas P. Burris ${ }^{9}$, Moshe Levi ${ }^{1}$

${ }^{1}$ Department of Biochemistry and Molecular \& Cellular Biology, ${ }^{2}$ Tauber Bioinformatics Research Center, University of Haifa, Mount Carmel, Haifa, 31905, Israel; ${ }^{3}$ Sagol Department of Neurobiology, University of Haifa, Mount Carmel, Haifa, 31905, Israel; ${ }^{4}$ Laboratory of Metabolism, Center for Cancer Research, National Cancer Institute, National Institutes of Health, Bethesda, MD 20892, USA; ${ }^{5}$ Department of Pharmacology and Physiology, Georgetown University, Washington DC 20057, USA; ${ }^{6}$ Thoracic and GI Malignancies Branch, National Cancer Institute, National Institutes of Health, Bethesda, MD 20814, USA; ${ }^{7}$ Office of Research Services, Office of the Director, National Institutes of Health, Bethesda, MD 20892, USA; ${ }^{8}$ Department of Pathology, Johns Hopkins University School of Medicine, Renal Pathology Service, Baltimore, MD 21287, USA; ${ }^{9}$ Center for Clinical Pharmacology, Washington University School of Medicine and St. Louis College of Pharmacy, St. Louis, MO 63110, USA; ${ }^{10}$ Department of Pharmacology \& Physiology, Saint Louis University School of Medicine, St. Louis, MO 63104, USA; ${ }^{11}$ Kidney Diseases Section, National Institute of Diabetes and Digestive and Kidney Diseases, National Institutes of Health, Bethesda, MD 20814, USA

Correspondence:

Moshe Levi

Department of Biochemistry and Molecular \& Cellular Biology, Georgetown University,

3900 Reservoir Road, Basic Science 353,

Washington DC 20057, USA

Tel: 202-687-9296

E-mail: Moshe.Levi@georgetown.edu 


\section{Running title: ERR in aging kidney}

\section{Significance Statement}

There is an increasing need for prevention and treatment strategies for age-related kidney disease. The hallmarks of aging kidneys are decreased mitochondrial function and increased inflammation. The expression of the nuclear hormone receptors estrogen-related receptors (ERRs) are decreased in aging human and mouse kidneys. This paper investigates the role of ERRs in the aging kidney. Treatment of aging mice with a pan-ERR agonist reversed the age-related increases in albuminuria and podocyte loss, mitochondrial dysfunction and inflammatory cytokines, including the cGAS-STING signaling pathways. Treatment of aging mice with a STING inhibitor decreased inflammation and increased mitochondrial gene expression. These findings identify ERRs as important modulators of age-related mitochondrial dysfunction and inflammation. 


\section{ABSTRACT}

Background: A gradual decline in renal function occurs even in healthy aging individuals. In addition to aging per se, concurrent metabolic syndrome and hypertension, which are common in the aging population, can induce mitochondrial dysfunction and inflammation, which collectively contribute to age-related kidney dysfunction and disease. Here we studied the role of the nuclear hormone receptors, the estrogen-related receptors (ERRs) in regulation of age-related mitochondrial dysfunction and inflammation. ERRs were decreased in aging human and mouse kidneys and were preserved in aging mice with lifelong caloric restriction (CR).

Methods: A pan-ERR agonist was used to treat 21-month-old mice for 8-weeks. In addition, 21-month-old mice were treated with a STING inhibitor for 3 weeks.

Results: Remarkably, only an 8-week treatment with a pan-ERR agonist reversed the age-related increases in albuminuria, podocyte loss, mitochondrial dysfunction and inflammatory cytokines, including the cGAS-STING and STAT3 signaling pathways. A 3-week treatment of 21-month-old mice with a STING inhibitor reversed the increases in inflammatory cytokines and the senescence marker p21 but also unexpectedly reversed the age-related decreases in PGC-1a, ERRa, mitochondrial complexes and MCAD expression.

Conclusions: Our studies identified ERRs as important modulators of age-related mitochondrial dysfunction and inflammation. These findings highlight novel druggable pathways that can be further evaluated to prevent progression of age-related kidney disease. 


\section{INTRODUCTION}

The fastest growing segment of the US population with impaired kidney function is the 65 and older age group. This population is expected to double in the next 20 years, while the number worldwide is expected to triple from 743 million in 2009 to 2 billion in 2050. This will result in a marked increase in the elderly population with chronic kidney disease. This increase may be further amplified by other age-related comorbidities including metabolic syndrome and hypertension that accelerate age-related decline in renal function ${ }^{1}$. Thus, there is a growing need for prevention and treatment strategies for age-related kidney disease.

A gradual age-related decline in renal function occurs even in healthy aging individuals ${ }^{2}$. Progressive glomerular, vascular and tubulointerstitial sclerosis is evident on renal tissue examination of healthy kidney donors with increasing age ${ }^{3}$. In addition to aging per se, metabolic syndrome and hypertension can induce mitochondrial dysfunction and inflammation, as well as endoplasmic reticulum stress, oxidative stress, altered lipid metabolism, and elevation of profibrotic growth factors in the kidney, which collectively contribute to age-related kidney disease ${ }^{2}$.

There is variation in the rate of decline in renal function as a function of sex, race, and burden of co-morbidities ${ }^{4-6}$. Interestingly, examination of processes leading to sclerosis suggests a role for possible modifiable systemic metabolic and hormonal factors that can ameliorate the rate of sclerosis. With the population of older individuals increasing, identifying preventable or treatable aspects of age-related nephropathy becomes of critical importance. 
There is increasing evidence that mitochondrial biogenesis, mitochondrial function, mitochondrial unfolded protein response (UPR $\left.{ }^{\mathrm{mt}}\right)$, mitochondrial dynamics, and mitophagy are impaired in aging, and these alterations contribute to the pathogenesis of the age-related diseases ${ }^{7-9}$. In this regard current studies are concentrated on modulating these molecular mechanisms to improve mitochondrial function.

Caloric restriction (CR) plays a prominent role in preventing age-related complications. We have previously shown that CR prevents the age-related decline in renal function and renal lipid accumulation via, at least in part, inhibition of the increased activity of the sterol regulatory element binding proteins (SREBPs) ${ }^{10,11}$. CR is also an important modulator of mitochondrial function. We have demonstrated that CR prevents age-related mitochondrial dysfunction in the kidney by increasing mitochondrial/nuclear DNA ratio, mitochondrial complex activity including fatty acid $\beta$-oxidation and expression of the mitochondrial transcription factor NRF1, the protein kinase AMPK, the deacetylases sirtuin 1 and 3 , and mitochondrial isocitrate dehydrogenase (IDH2) expression ${ }^{12}$. In addition, CR also prevents the age-related decrease in mitochondrial number in the renal tubules. CR increases expression of the bile acid regulated nuclear receptor farnesoid $X$ receptor $(F X R)$ and the Takeda $G$ protein coupled receptor (TGR5). Treatment of 22-month-old ad lib (AL)-fed mice for 2 months with the dual FXR-TGR5 agonist INT-767 reversed most of the age-related impairments in mitochondrial function and the progression of renal disease ${ }^{12}$. Importantly INT-767 and CR each increased expression of PGC-1a, ERR $\alpha$, and ERR $\gamma$, which are important regulators of mitochondrial biogenesis and function. 
The estrogen-related receptors (ERRs) ERR $\alpha$ (NR3B1, ESSRA gene), ERR $\beta$ (NR3B2, ESRRB gene), and ERR $\gamma$ (NR3B3, ESRRG gene) are members of the nuclear receptor superfamily. While one report suggests a role for cholesterol ${ }^{13}$, there are no confirmed endogenous ligands for these orphan receptors. Importantly the ERRs do not bind natural estrogens, and they do not directly participate in classic estrogen signaling pathways or biological processes ${ }^{14}$. ERRa and ERR $\gamma$ are strongly activated by their coactivators PGC-1 $\alpha$ and PGC-1 $\beta^{15}$. In contrast, RIP140 and NCoR1 are important corepressors of ERR activity ${ }^{16,17}$. ERRs are subject to post-translational modifications including phosphorylation, sumoylation, and acetylation that modulate their DNA binding and recruitment of coactivators ${ }^{18,19}$.

$E R R a$ and ERR $\gamma$ regulate the transcription of genes involved in mitochondrial biogenesis, oxidative phosphorylation, tricarboxylic acid (TCA) cycle, fatty acid oxidation and glucose metabolism ${ }^{14}$. However, in addition to overlapping gene activation there is also ample evidence that ERRa and ERR $\gamma$ have differential and opposing effects, which can be due to interactions with corepressors, coactivators, posttranslational modification, or differential cell expression ${ }^{14}$. Opposing effects for ERRa and ERR $\gamma$ are seen in breast cancer ${ }^{20}$, regulation of gluconeogenesis in the liver ${ }^{20,21}$, skeletal muscle function ${ }^{20,21}$, macrophage function ${ }^{21,22}$, and regulation of lactate dehydrogenase $\mathrm{A}$ $(\mathrm{LDHA})$ related to anaerobic glycolysis ${ }^{14}$.

ERRa and ERR $\gamma$ are highly expressed in the mouse and human kidney ${ }^{23,24}$. However, the roles of ERRa and ERR $\gamma$ in modulating age-related impairment of mitochondrial function and age-related inflammation (inflammaging) are not known. We undertook our current studies to determine whether a pan-ERR agonist, activating 
bioRxiv preprint doi: https://doi.org/10.1101/755801; this version posted July 1,2020 . The copyright holder for this preprint (which was not certified by peer review) is the author/funder, who has granted bioRxiv a license to display the preprint in perpetuity. It is made available under aCC-BY-NC-ND 4.0 International license.

ERRa, ERR $\beta$, and ERR $\gamma$, would improve mitochondrial function and inflammation in the aging mouse kidney. 


\section{METHODS}

Mice: Studies were performed in 4-month-old and 21-month-old male C57BL/6 male mice obtained from the NIA aging rodent colony. Mice received 3\% DMSO vehicle or the pan-ERR agonist SLU-PP-332 (Dr. Thomas Burris, Washington University) at a dose of $25 \mathrm{mg} / \mathrm{kg}$ body weight/day, administered intraperitoneally. Mice were dosed for 8 weeks, following which they underwent euthanasia and the kidneys were harvested and processed for a) histology, b) transmission electron microscopy, c) isolation of nuclei, d) isolation of mitochondria, and e) biochemical studies detailed below. Another cohort of male C57BL/6 mice with the same age were received the same vehicle as above or the STING inhibitor C-176 (Focus Biomolecules, Plymouth Meeting, PA) at a dose of $1 \mathrm{mg} / \mathrm{kg}$ body weight/day for 3 weeks via intraperitoneal injection.

Immunohistochemistry: Formalin-fixed paraffin-embedded tissue sections were subjected to antigen retrieval with EDTA buffer in high pressure heated water bath and staining was performed using either mouse monoclonal ERRa (1:2500, Abcam, Cambridge, MA) or ERRy (1:400, Abcam) antibodies for 90 minutes or pyruvate dehydrogenase (PDH) E2/E3bp (1:1000, Abcam) antibody for 45 minutes. UnoVue HRP secondary antibody detection reagent (Diagnostic BioSystems, Pleasanton, CA) was applied followed by DAB chromogen. Imaging was done with Nanozoomer (Hamamatsu Photonics, Japan). 
Cell culture: Primary human proximal tubule epithelial cells (cat\#: PCS-400-010) were purchased from ATCC (Manassas, VA). Cells were cultured in Renal Epithelial Cell Basal Medium (ATCC cat\#: PCS-400-030) supplemented with Renal Epithelial Cell Growth Kit (ATCC cat\#: PCS-4000-040) at $37^{\circ} \mathrm{C}$ in 5\% CO2. Cells were cultured to 70$80 \%$ confluence and then treated with vehicle $(4 \mathrm{mM} \mathrm{HCl}$ with $0.1 \%$ bovine serum albumin) or 10 ng/mL TGF- $\beta 1$ (Cat\#: 7754-BH-005, R\&D Systems, Minneapolis, MN) or 10 ng/mL TNF-a (Cat\#210-TA-020, R\&D Systems) for 24 hours. Cells were then harvested and analyzed for gene expression.

Transmission Electron Microscopy: One $\mathrm{mm}^{3}$ cortex kidney tissues were fixed for 48 hrs. at $4^{\circ} \mathrm{C}$ in $2.5 \%$ glutaraldehyde and $1 \%$ paraformaldehyde in $0.1 \mathrm{M}$ cacodylate buffer $(\mathrm{pH} 7.4)$ and washed with cacodylate buffer three times. The tissues were fixed with $1 \%$ $\mathrm{OsO}_{4}$ for two hours, washed again with $0.1 \mathrm{M}$ cacodylate buffer three times, washed with water and placed in 1\% uranyl acetate for one hour. The tissues were subsequently serially dehydrated in ethanol and propylene oxide and embedded in EMBed 812 resin (Electron Microscopy Sciences, Hatfield, PA). Thin sections, approximately $80 \mathrm{~nm}$, were obtained by utilizing the Leica ultracut-UCT ultramicrotome (Leica, Deerfield, IL) and placed onto 300 mesh copper grids and stained with saturated uranyl acetate in 50\% methanol and then with lead citrate. The grids were viewed with a JEM-1200EXII electron microscope (JEOL Ltd, Tokyo, Japan) at 80kV and images were recorded on the XR611M, mid mounted, 10.5M pixel, CCD camera (Advanced Microscopy Techniques Corp, Danvers, MA). Mitochondrial morphology was assessed with ImageJ software by manually tracing all mitochondria that were completely within 
the field of view in 6 random images from each mouse ( $n=3-4$ each group). The area, perimeter, and minimum Feret diameter of each mitochondrion were measured.

Autofluorescence FLIM: autofluorescence FLIM signals were acquired using an Olympus FVMPERS microscope (Waltham. MA) equipped with the DIVER (Deep Imaging via Enhanced-Photon Recovery) detector which was developed at the Laboratory for Fluorescence Dynamics (LFD), University of California at Irvine, CA, and FLIMBox (ISS, Champaign, IL) for phasor lifetime acquisition, as detailed in

\section{Supplementary Methods.}

RNA extraction and real-time quantitative PCR: Total RNA was isolated from the kidneys using Qiagen RNeasy mini kit (Valencia, CA), and cDNA was synthesized using reverse transcript reagents from Thermo Fisher Scientific (Waltham, MA). Quantitative real-time PCR was performed as previously described ${ }^{25}$, and expression levels of target genes were normalized to $18 \mathrm{~S}$ level. Primer sequences are listed in

\section{Supplementary Table 1.}

Bulk RNA-seq: Approximately 300-500 ng of kidneys RNA were used to generate barcoded RNA libraries using Ion AmpliSeq Transcriptome Mouse Gene Expression Panel, Chef-Ready Kit. Library quantification was performed using the lon Library Quantitation Kit (Thermo Fisher Scientific). Sequencing was done on an Ion Proton with signal processing and base calling using lon Torrent Suite (Thermo Fisher Scientific). Raw reads were mapped to Ampliseq supported mm10 transcriptome. Normalized read 
counts per million were generated using the RNA-seq Analysis plugin (Ion Torrent Community, Thermo Fisher Scientific). Gene expression table was converted to LN scale and quantile normalized. Genes with expression levels less than 4 across all samples were filtered out. Selection of the differentially expressed genes was performed utilizing student t-test. The significance of differentiation was defined as Student t-test statistic $p$-value $<0.05$. Enrichment analysis of $\mathrm{GO}$ biological processes and pathways was prepared with DAVID ${ }^{26}$ and with PANTHER online tool ${ }^{27}$. Heat map visualization was performed with Heatmapper tool ${ }^{28}$.

Single nuclei RNA-seq: Mouse kidney single nuclei were isolated ${ }^{29,30}$ and counted using the EVE Automated Cell Counter (NanoEnTek, VWR, Radnor, PA). The resulting mixture was provided to the Genomics and Epigenomics Shared Resource (GESR) at Georgetown University, and further processed by the Chromium Controller (10X Genomics, Pleasanton, CA) using Single Cell 3' GEM Kit v3, Single Cell 3' Library Kit v3, i7 multiplex kit, Single Cell 3' Gel Bead Kit v3 (10X Genomics) according to the manufacturer's instructions with modifications for single nuclei. Libraries were sequenced on the Illumina Novaseq S4 System (Illumina, San Diego, CA) to an average depth of $>300 \mathrm{M}$ reads PF per sample. Sequencing data were processed by Cellranger pipeline. Briefly, reads were aligned against the mouse mm10 genome reference with STAR algorithm. Barcodes and UMIs were filtered and corrected. Only confidently mapped, non-PCR duplicates with valid barcodes and UMls were used to generate a gene-barcode matrix for further analysis. Expression matrix was further investigated by the Loupe Cell Browser. 
Proteomics: 200 microgram of kidney tissue were homogenized and lysed by 8M urea in 20mM HEPES ( $\mathrm{pH}=8.0)$ buffer with protease and phosphatase inhibitors using Tissue Lyser II (QIAGEN). Samples were reduced and alkylated followed by MS-grade trypsin digestion. The resulting tryptic peptides were labeled with 11 plex tandem mass tag (TMT). After quench, the tagged peptides were combined and fractionated with basic$\mathrm{pH}$ reverse-phase high-performance liquid chromatography, collected in a 96-well plate and combined for a total of 12 fractions prior to desalting and subsequent liquid chromatography-tandem mass spectrometry (LC-MS/MS) processing on a Orbitrap QExactive HF (Thermo Fisher Scientific) mass spectrometer interfaced with an Ultimate 3000 nanoflow LC system ${ }^{31}$. Each fraction was separated on a reverse phase $C_{18}$ nano-column $(25 \mathrm{~cm} \times 75 \mu \mathrm{m}, 2 \mu \mathrm{m}$ particles $)$ with a linear gradient $4 \sim 45 \%$ solvent $B$ (0.1\% TFA in Acetonitrile). Data dependent mode was applied to analyze the top 15 most abundant peaks in one acquisition cycle.

MS raw files were mapped against Uniprot mouse database (version 20170207) using the MaxQuant software package (version 1.5.3.30) with the Andromeda search engine 32. Corrected intensities of the reporter ions from TMT labels were obtained from the MaxQuant search. The normalized relative abundance of a protein in each sample was calculated as the ratio of a protein abundance in a sample to abundance of the same protein in a pool (channel 11, pooled from 20 samples) of the corresponding batch. After performing principle component analysis (PCA) on the protein relative abundance matrix we noticed that the samples were still separated by batches on the PC1-PC2 
plane. Thus, we further corrected this batch effect by applying the Experimental Bayes (EB) batch correction method ${ }^{33}$. After batch correction, PCA revealed clear separation of samples into biological groups on the PC1-PC2 plane (Supplementary Figure 1). All further analyses were performed on batch corrected protein abundance matrix.

Selection of the differentially abundant proteins was done utilizing Wilks theorem based likelihood ratio test ${ }^{34}$ and Student t-test statistic. The significance of differentiation was defined as likelihood ratio statistic $p$-value $<0.001$ and Student t-test statistic $p$-value $<$ 0.05 .

Mitochondrial isolation: Kidney mitochondria were isolated using the kit (MITOISO1) from Sigma (St. Louis, MO) according to manufacturer instructions.

Mitochondrial Biogenesis: We measured mitochondrial (Cytb) and nuclear (H19) DNA by RT-QPCR.

Mitochondrial Respiration: We measured basal respiration, ATP turnover, proton leak, maximal respiration and spare respiratory capacity using the Seahorse XF96 Analyzer on equally loaded freshly isolated kidney mitochondria. We also measured mitochondrial complex I, II, III, IV, and V protein abundance by Native Blue Gel Electrophoresis (Thermo Fisher Scientific) with equally loaded mitochondrial fractions.

\section{Multi-omics data analysis and integration bioinformatics methods: Taking} advantage of the multi-omics (transcriptomics and proteomics) measurements reflecting 
the same biological processes in the samples, we performed Two-way Orthogonal Partial Least Square (O2PLS) integration ${ }^{35}$ to identify networks of associated genes and proteins. This analysis identified one orthogonal component (V2) that reflected the expected separation of samples into three distinct groups: young together with young pan-ERR agonist treated mice, old mice, and old pan-ERR agonist treated mice. We identified a subset of genes and proteins with V2 highest loadings (threshold was arbitrarily set to 0.04 or lower than -0.04$)$ for further analysis.

Western blotting: Western blotting was performed as previously described ${ }^{25,36-38}$. Equal amount of total protein was separated by SDS-PAGE gels and transferred onto PVDF membranes. After HRP-conjugated secondary antibodies, the immune complexes were detected by chemiluminescence captured on Azure C300 digital imager (Dublin, CA) and the densitometry was performed with ImageJ software. Primary antibodies used for western blotting were listed in Supplementary Table 1.

Cytokine arrays: Cytokines in 200 microgram kidney lysate (pooled from 5-6 samples with equal amount of protein) were detected with the Proteome Profiler Array (ARY028; R\&D Systems) according to manufacturer's instructions.

Statistical analysis: Results are presented as the means \pm SEM for at least three independent experiments. Following the Grubbs' outlier test, the data were analyzed by ANOVA and Newman-Keuls tests for multiple comparisons or by $t$ test for unpaired 
data between two groups (Prism 6, GraphPad, San Diego, CA). Statistical significance was accepted at the $P<0.05$ level.

Study approval: Animal studies and relative protocols were approved by the Animal Care and Use Committee at the Georgetown University. All animal experimentation was conducted in accordance with the Guide for Care and Use of Laboratory Animals (National Institutes of Health, Bethesda, MD). 


\section{RESULTS}

\section{ERR $\alpha$ and ERR $\gamma$ expression is decreased in the aging human kidney}

In a previous study, we found decreased expression of ERRa and ERR $\gamma$ in the aging mouse kidney and expression was reversed by the dual FXR-TGR5 agonist INT-767 or CR. Further, increased ERR expression correlated with increased mitochondrial biogenesis and function in the treated aging kidneys ${ }^{12}$. In light of the role of ERR in mitochondrial biogenesis, we determined whether decreased expression also occurs in the aging human kidney. We performed immunohistochemistry with human kidney sections from young and old individuals. The results indicate that both $\operatorname{ERR} \alpha$ and $E R R \gamma$ are expressed in renal tubules and that their expression levels were markedly decreased in aging human kidney (Figure 1A).

Since ERRs are important modulators of mitochondrial biogenesis, we also stained the human kidney sections for the mitochondrial pyruvate dehydrogenase (PDH) e2/e3 and found a marked decrease in PDH immunostaining in the aging human kidney samples (Figure 1B).

TGF- $\beta$ and TNF- $\alpha$ mediate decreases in ERR $\gamma$ in cultured human proximal tubular epithelial cells

Transforming growth factor- $\beta$ (TGF- $\beta$ ) and tumor necrosis factor- $\alpha$ (TNF- $\alpha$ ) expression are increased in the aging kidney (Figure 1C-D). Thus, we determined whether TGF- $\beta$ and TNF- $\alpha$ at least in part mediate the decreased expression of ERR in the kidney. We 
found that in cultured human primary proximal tubular cells (PTEC), TGF- $\beta$ significantly decreased expression level of ERR $\gamma$ mRNA while ERRa was unaffected. Expression of PGC1a, MCAD (encoding medium chain acyl-CoA dehydrogenase), SOD2, and UCP2 were also decreased in TGF- $\beta$ treated cells (Figure 1C).

TNF- $\alpha$ also had a similar effect to decrease PGC1a, ERR $\gamma$, SOD2, and UCP2 expression (Figure 1D).

\section{ERR $\alpha$ and ERR $\gamma$ RNA distribution in the mouse kidney}

To determine where ERRa and ERR $\gamma$ mRNA are expressed in the mouse kidney, we

performed single nuclei RNAseq ${ }^{29,30}$. With 3,000-5,000 nuclei sequenced at $100 \mathrm{k}$ read depth, 12 expression clusters were identified and assigned to major cell types in the mouse kidney (Figure 2A). We found that ERRa was expressed in most of proximal tubules, intercalated cells and podocytes. ERR $\gamma$ was detected in much less cells and mainly appeared in proximal tubules and intercalated cells (Figure 2B). Compared to young kidneys, S1/S2 segments of aging proximal tubules showed a decline in both ERRa and ERR $\gamma$ expression (Figure 2C).

\section{Pan-ERR agonist treatment improves the age-related kidney injury}

We treated the aging mice with a recently available pan-ERR agonist (SLUPP-332). We found that 2-month treatment of 21-month-old mice significantly improved the agerelated albuminuria, and decreased kidney weight (Figure 3A). The decrease in albuminuria is likely related to improved podocyte function as protein expression of the 
podocyte marker NPHS2 (podocin) is increased after the treatment (Figure 3B). In addition, mRNA expression in profibrotic markers (TGF- $\beta$, PAl-1 and Col IV), monocyte/macrophage marker (F4/80) and tubular injury marker neutrophil gelatinaseassociated lipocalin (NGAL) were decreased with the pan-agonist treatment (Figure 3C). Finally, with cytokine profiling we found the kidney injury-related proteins, Ngal, kidney injury marker-1 (Kim1), osteopontin and CC-motif ligand chemokine-21 (Ccl21) were increased in the aging kidney and partially normalized by pan-ERR agonist treatment (Figure 3D).

\section{Pan-ERR agonist treatment modulates mitochondrial metabolism and inflammation in the aging kidney}

To assess the actions of the pan-ERR agonist, we examined ERR $\alpha$, ERR $\beta$ and ERRy mRNA abundance. Although they were all decreased in the kidneys of aging mice, treatment with the ERR pan-agonist induced significant increases in their expression to levels observed in the young mice (Figure 4A).

Next, we performed bulk mRNA sequencing analysis to determine which pathways are affected by aging and are restored by pan-ERR agonist. We first investigated the molecular changes in aging kidney. We found 448 upregulated and 463 downregulated genes in kidneys of aging mice compared to young controls (Supplementary Figure 2A, Supplementary Table 2). Pathway enrichment analysis of upregulated genes revealed that inflammation-related pathways were highly significant. The inflammationrelated pathways included 'Inflammation mediated by chemokine and cytokine signaling 
pathway', 'EGF receptor signaling pathway', 'Toll receptor signaling pathway', and 'TGF-beta signaling pathway' which were all previously reported as upregulated in aging kidney $^{39,40}$ (Supplementary Figure 2B).

In addition to the inflammation, apoptosis and cell-cycle regulating pathways were also enriched with upregulated genes in the aging kidney (Supplementary Figure 2B). The cell-cycle dysregulation is a known phenomenon in the aging and diseased kidney and might indicate cell senescence ${ }^{41}$. Indeed, when we analyzed the RNA-seq data in a supervised manner identifying senescence associated genes, we found that in aging kidney 29 senescence associated genes were upregulated and 10 were downregulated (Supplementary Figure 2C, Supplementary Table 3). In particular, we found that p53 (Trp53), p65 (Rela), and p21(Cdkn1a) which are the major indicators of senescent cells were highly expressed in aging kidney. The downregulated genes in aging kidney were enriched in mitochondrial and metabolic processes (Supplementary Figure 2D). Dysregulated mitochondrial functioning has been widely studied in aging kidneys ${ }^{42}$. Recently, several studies have shown that damaged mitochondria may trigger inflammation via cGAS-STING pathway in kidney disease ${ }^{43,44}$. TFAM is a key regulator of mitochondrial gene expression ${ }^{45}$ and is crucial for maintaining mtDNA structure, transcription, and replication ${ }^{46}$. Deletion of Tfam leads to mtDNA escape into the cytoplasm and activation of the innate immune pathway through cGAS-STING activation $^{44}$. Our mRNA sequencing data indicates that Tfam mRNA levels are decreased in aging kidney (Supplementary Figure 2E). Overall, we found that in aging kidney there is a dysregulation of mitochondrial and immune processes. 
We next examined the effect of pan-ERR agonist treatment on gene expression. PanERR agonist treatment mitigated several of the abovementioned pathways (Figure 4B-

\section{C, Supplementary Table 4).}

In addition to mRNA sequencing, we performed mass-spec proteomics. Because the concordance of proteomics and RNA transcriptomics is known to be weak ${ }^{47,48}$, we expected the proteomics analysis to reveal additional processes disrupted in aging and attenuated by pan-ERR agonist treatment. We found that, on the protein level, mitochondria-related, oxidation reduction processes, peroxisome, and metabolic pathways were dysregulated in aging kidney and correlated with pathways found on mRNA level. Furthermore, several additional dysregulated pathways were identified from proteomics analysis in aging kidney. These additional processes included calcium binding region, blood coagulation, and biotin/lipoyl attachment processes (Supplementary Figure 2F-G, Supplementary Table 5). The processes of mitochondrion, immune-related pathways, transport regulation, focal adhesion, and fatty acid oxidation were improved by pan-ERR agonist treatment (Figure 4D-E,

\section{Supplementary Table 6).}

It has been suggested that combining several layers of omics data yields clearer understanding of complex biological phenomena than evaluation of each layer separately ${ }^{49,50}$. Thus, applying the two-way orthogonal Partial Least Squares (o2PLS)

51 , we found that one of the components (V2) reflects the expected relation between the 
biological groups under investigation. Namely, V2 component profile is associated with pan-ERR agonist effect of alleviating the dysregulation of genes and proteins in aging kidneys (Supplementary Figure 3). Genes and proteins whose expression correlates with V2 component profile were identified as an interrelated molecular network associated with overall changes in aging and pan-ERR agonist treatment (see Methods, Supplementary Table 7). We found that the subset of genes and proteins upregulated in aging kidney and downregulated by pan-ERR agonist treatment were enriched with immune related processes such as antigen processing and presentation, complement and coagulation cascades, Ig-like C1-type domain protein activation, and TNF signaling pathway (Figure 4F). The subset of genes and proteins downregulated in aging kidney and enhanced by pan-ERR agonist treatment was enriched by processes associated with metabolism such as lipid metabolic processes, fatty acid metabolism, and catalytic activity (Figure 4G).

Taken together, our analyses of transcriptomic and proteomic changes as well as integrated analyses of transcriptome-proteome associations point to downregulation of mitochondrial metabolism and upregulation of inflammatory processes in aging kidney. Theses analyses also showed that pan-ERR agonist treatment was effective in attenuating these age-related dysregulations. Thus, our analyses correlate with previous studies to show that the hallmarks of aging kidneys are decreased mitochondrial function and increased inflammation ${ }^{52,53}$. We next determined whether treatment with the pan-ERR agonist improved these specific defects in the aging kidneys. 


\section{Pan-ERR agonist treatment restored mitochondrial function in aging kidneys}

The canonical function of ERR is to induce mitochondrial biogenesis. We found that the pan-ERR agonist increased expression of the master mitochondrial biogenesis regulators PGC1 $\alpha$ and $P G C 1 \beta$ in aging kidneys. Consistently, mitochondrial transcription factor Tfam1 expression was decreased in the aging kidney and increased with the pan-ERR agonist. As a result, the mitochondrial DNA/nuclear DNA ratio was increased in aging kidneys following treatment, indicative of increased mitochondrial biogenesis (Figure 5A). The increased mitochondrial biogenesis was accompanied by increased expression of genes related to the mitochondrial ETC complexes, such as complex I subunit Ndufb8, complex II subunit Sdhc, complex III subunit Uqcrb, complex IV subunit Cox6a2, and complex V subunit Atp5b (Figure 5B). Native blue gel analysis further showed increased quantities of assembled mitochondrial complexes after the treatment (Supplementary Figure 4). This is consistent with the increased gene expression of enzymes in TCA cycle, such as Pdhb, Mdh1, Idh3b, and Sucla2 (Figure 5C). Since the TCA cycle can produce NADH which is required for complex I driven mitochondrial oxidative phosphorylation, we further determined fractional intensity of free $\mathrm{NADH}$ with phasor approach to fluorescence lifetime imaging microscopy (FLIM) ${ }^{54}$, ${ }^{55}$. Analysis of the FLIM images showed that there was decreased free NADH fraction in aging kidneys, which suggests a lower capacity of NADH regeneration in aging kidney, in agreement with the downregulation of TCA cycle enzyme expression ${ }^{56}$. Treatment with the pan-ERR agonist shifted the free $\mathrm{NADH}$ fraction in the aging kidney towards that seen in the young kidneys as shown in cumulative plots for all samples ${ }^{55,57}$ (Figure 5D). 
The interrelationship between the ETC and TCA cycle is illustrated in Figure 5E. The changes in ETC complexes and TCA cycle resulted in increased maximum respiration capacity in mitochondria isolated from treated aging kidneys (Figure 5F). In addition, mRNAs encoding enzymes that mediate mitochondrial fatty acid $\beta$-oxidation, including Cpt1a and Mcad, were upregulated by the pan-ERR agonist (Figure 5G), suggesting that ERR agonism promotes mitochondrial fatty acid $\beta$-oxidation.

\section{Pan-ERR agonist treatment altered mitochondrial dynamics in aging kidneys}

Transmission electron microscopy showed alterations in the mitochondria of aging kidneys, including decreases in the area, perimeter, and minimum Feret diameter in the aging kidneys; these parameters were restored to levels seen in young kidneys upon treatment with the pan-ERR agonist (Figure 6A). Since these mitochondrial changes are reminiscent of alterations in mitochondrial fusion and fission, we also measured the expression of proteins that regulate mitochondrial fusion and fission. Mitofusin 2 (Mfn2) is found in the outer membrane that surrounds mitochondria and participates in mitochondrial fusion ${ }^{58}$. There was a significant decrease in Mfn2 in the aging kidney, and the ERR pan agonist increased the Mfn2 protein abundance in both the young and the old kidneys, fully restoring levels in the old kidneys to levels of the untreated young kidneys (Figure 6B).

There were changes in expression of other nuclear-encoded, mitochondrial-expressed mRNAs and proteins that would be congruent with restoration of mitochondrial function. Opa1 protein localizes to the inner mitochondrial membrane and helps regulate 
mitochondrial stability, energy output, and mitochondrial fusion ${ }^{59}$. While there was no significant change in the protein level in the aging kidney, upon treatment there was a tendency for the protein level to increase in the aging kidneys (Figure 6B). In addition, there were also significant decreases in mitoguardin 2 (Miga2) and mitoPLD (Pld6) mRNA levels in the aging kidneys that were increased upon treatment with the panERR agonist (Figure 6B). Mitoguardin 2 regulates mitochondrial fusion through mitoPLD ${ }^{60}$. Drp1 is a member of the dynamin superfamily of proteins and is a fundamental component of mitochondrial fission ${ }^{58}$. There were significant increases in Drp1 and phospho-Drp1 protein in the kidneys of aging mice, which were restored to levels seen in young mice following treatment with the pan-ERR agonist (Figure 6B).

\section{Pan-ERR agonist treatment decreased inflammation in aging kidneys}

Mitochondria are immunogenic organelles and mitochondrial dysfunction generates several immunogenic molecules, including $m t^{2} N A^{61}$ and $m t R N A^{62}$. The cyclic GMPAMP synthase (cGAS)-stimulator of interferon genes (STING) has been reported as one of the innate immune receptors to be activated by mtDNA leaking into cytosol ${ }^{44,63}$. In the aging kidneys, we found increased expression of STING and cGAS mRNAs and proteins, which was significantly reversed by treatment with the pan-ERR agonist (Figure 7A). We also found similar changes with RNA sensors RIG-I/MDA5/LGP2 and other nucleic acid sensors, such as TLRs ${ }^{64,65}$ (Figure 7B). We further examined the downstream response and we found out that expression of mRNA encoding components in NFkB signaling pathway (Rel, RelB, Nfkb2, and p65 protein) were increased in the aging kidney, and reduced by pan-ERR agonist treatment (Figure 7C). 
In addition, we examined another potential STING-activated interferon signaling pathway and observed the corresponding changes in STAT3 (Figure 7C). We found significant increases in both total STAT3 and p-Tyr705-STAT3 protein, a marker for STAT3 activation. These increases were substantially suppressed upon treatment with the pan-ERR agonist. This cascade signaling activation was in parallel to the expression of senescence marker p21, which was induced in the aging kidney and reduced by the treatment with the pan-ERR agonist (Figure 7D). Another senescence marker, p16, was increased in the aging kidneys but was unaffected by the treatment (Figure 7D). Both of these cellular senescence markers are downregulated in aging kidneys with lifelong CR (Supplementary Figure 5).

To determine which senescence associated secretory phenotype (SASP) factors are regulated by the pan-ERR agonist treatment in the aging kidney, we searched the RNAseq data and verified by real-time PCR that RNAs encoding the proinflammatory cytokines IL-1 $\beta$ and TNF- $\alpha$, the chemokine receptor CCR5, the metallopeptidase inhibitor TIMP1, and the cell adhesion molecule ICAM1 were increased in the aging kidney and treatment with the pan-ERR agonist decreased their expression (Figure 7D).

\section{STING inhibitor decreased inflammation and increased mitochondrial gene expression in aging kidneys}

To determine whether the increase in STING mRNA and protein per se mediates agerelated increase in inflammation, we treated aging mice with a known STING inhibitor C$176^{66}$. C-176 decreased IL-1 $\beta$, Stat3, phospho-Stat3 and the senescence marker p21 expression in the aging kidney (Figure 8A). Unexpectedly, we found the expression of 
master regulator for mitochondria biogenesis $P G C 1 \alpha$ and $P G C 1 \beta$ in aging kidney were also increased with the treatment, along with ERRa (Figure 8B). Genes involved in mitochondrial ETC complex, Ndufs1, Cox6a2, and ATP5e, and fatty acid oxidation gene Mcad were also found upregulated by the STING inhibitor (Figure 8B).

Interestingly, in proximal tubular epithelial cells, TNF-a, the proinflammatory cytokine which is increased in the aging kidney, induced impairments in genes that mediate mitochondrial biogenesis and the ETC complex (Figure 1D). These results suggest that in aging, inflammation and mitochondrial dysfunction may regulate each other to amplify age-related kidney disease. 


\section{DISCUSSION}

The data presented here have identified the nuclear hormone receptors the estrogen related receptors $\mathrm{ERR} \alpha, \mathrm{ERR} \beta$, and $\mathrm{ERR} \gamma$ as important modulators of age-related mitochondrial dysfunction and inflammation. ERR $\alpha, E R R \beta$, and ERR $\gamma$ expression is decreased in the aging kidney, and lifelong CR results in increases in expression of $E R R \alpha, E R R \beta$, and ERR $\gamma$ in the kidney. In parallel, CR also prevents age-related mitochondrial dysfunction and inflammation ${ }^{12,67,68}$. ERRs therefore act as potential CR mimetics in the kidney. Remarkably, only an 8-week treatment of 21 -month-old mice with the pan-ERR agonist reversed the age-related increases in albuminuria and podocyte loss, mitochondrial dysfunction and inflammation. These effects were comparable with those achieved with lifelong $\mathrm{CR}$, which is known to protect against age-related co-morbidities, including loss of renal function ${ }^{10,11,67}$.

Recent evidence indicates that mitochondrial dysfunction is one of the mediators of cellular senescence, and the associated SASP includes pro-inflammatory cytokines and pro-fibrotic growth factors ${ }^{69,70}$. This process may also be involved in the age-related inflammation, termed inflammaging or senoinflammation, which is also prevented by CR 71,72

Our RNAseq data indicated that apoptosis and cell-cycle regulating pathways were also enriched in the aging kidney. Further analysis of this data in a supervised manner 
identified 29 senescence associated genes upregulated in the aging kidneys and 10 downregulated. In particular, we found that p53 (Trp53), p65 (Rela), and p21(Cdkn1a) which are the major indicators of senescent cells were highly expressed in aging kidney.

The downregulated genes in aging kidney were enriched in mitochondrial and metabolic processes based on our mRNA sequencing data. Dysregulated mitochondrial functioning has been widely studied in aging kidneys ${ }^{42}$. RNAseq data suggests that Tfam mRNA levels are decreased in aging kidney, which we have verified by RT-QPCR determination. TFAM is a key regulator of mitochondrial gene expression ${ }^{45}$ and is crucial for maintaining mtDNA structure, transcription, and replication ${ }^{46}$. It has been shown that deletion of Tfam leads to mtDNA escape into the cytoplasm and activation of the innate immune pathway through cGAS-STING activation which plays key roles in immunity, inflammation, senescence and cancer ${ }^{44,63,73,74}$. In addition to the recent identification of the importance of this signaling pathway in mouse models of acute kidney injury, chronic kidney disease and fibrosis ${ }^{43,44}$, our studies also show increased expression of STING in aging kidneys, and its downregulation following treatment with the pan-ERR agonist.

In addition to cGAS-STING signaling, mitochondrial dysfunction is also associated with activation of STAT3 ${ }^{75}$. Increased STAT3 signaling is associated with senescence ${ }^{76}$ as well as kidney disease ${ }^{77}$. We found increased STAT3 in the aging kidneys both at the mRNA and protein level, including increased phospho-STAT3 $\left(\mathrm{Tyr}^{705}\right)$ and normalization after treatment with the pan ERR agonist. 
To determine whether STING activation per se mediates aging-related inflammation, we treated aging mice with a STING inhibitor. We found that STING inhibition decreased inflammation in aging kidney, as reported in other models using a STING inhibitor or STING knockout mice ${ }^{43,44}$. However, we found that STING inhibition can further increase mitochondrial gene expression, suggesting a model where mitochondrial injury triggers inflammation, compounding mitochondrial dysfunction in aging kidney. Interestingly, in proximal tubular epithelial cells, TNF-a, the proinflammatory cytokine which is increased in the aging kidney, induced impairments in genes that mediate mitochondrial biogenesis and the ETC complex. These results suggest that in aging, inflammation and mitochondrial dysfunction may regulate each other to amplify agerelated kidney disease. Our findings are consistent with reports of mitochondrial damage in acute kidney injury induced by lipopolysaccharide (LPS), an endotoxin that activate innate immunity (via TLR4) to induce circulating cytokines ${ }^{78-80}$.

Overall, we found that in aging kidney there is a dysregulation of mitochondrial and immune processes. Pan-ERR agonist treatment mitigated several of the abovementioned pathways. In summary, our studies identify the ERRs as beneficial modulators of mitochondrial dysfunction and inflammation in the aging kidney. 
FIGURE LEGENDS

Figure 1. ERRa, ERRy, and PDH expression is decreased in the aging human kidney.

A) ERRa and ERRy immunohistochemistry on kidney sections from young and old subjects. The nuclear positive staining for ERRa or ERRy is decreased in old kidney sections. Box shows magnified image. B) PDH immunohistochemistry on kidney sections from young and old subjects. PDH staining is decreased in old kidney sections.

C-D) TGF- $\beta$ mRNA C) and TNFa protein expression D) were increased in the aging kidney. $n=4-6$ samples per group. TGF- $\beta 1$ C) and TNF $\alpha-D$ ) treatment of human primary proximal tubule cells (PTEC) significantly decreased expression levels of ERRy mRNA but not ERRa. PGC1a, MCAD, SOD2, and UCP2 gene expression were also significantly decreased in TGF- $\beta$ treated cells $\mathbf{C})$. TNF also had a similar effect to decrease PGC1a, ERRy, SOD2, and UCP2 expression D). N=3 for each group. ${ }^{* \star *} \mathrm{p}<$ 0.001 .

Figure 2. Single nuclei RNAseq of young and old kidneys.

A) The t-distributed stochastic neighbor embedding (tSNE) shows that, with 100k read depth and 3000-5000 nuclei sequenced, we were able to identify 12 clusters and assigned them to major cell types known in the mouse kidney. B) We found most ERRa is expressed in proximal tubules, intercalated cells and podocytes. For ERR $\gamma$, proximal tubules and intercalated cells expressed most strongly. The cells with positive expression of ERRa or ERR $\gamma$ were labeled purple. C) Table to show percentage of positive expressed cells in each cluster. Compared to the young kidneys, aging 
proximal tubules at S1/S2 show decline in ERRa and ERR $\gamma$ expression. Pod: podocyte; MC: mesangial cell; EC: endothelial cell; Mф: macrophage; DCT, distal convoluted tubule; LH, loop of Henle; IC: intercalated cell; CD-PC, collecting duct-principal cell; PT, proximal tubule.

\section{Figure 3. Pan-ERR agonist improved age-related renal injury.}

A) Albuminuria and kidney weight (normalized by body weight) were increased in aging kidneys and normalized with treatment. $\mathrm{N}=5-6$ for each group. B) NPHS2 (podocin) immunohistochemistry on kidney sections, labeling podocytes. Pan-ERR agonist treatment reversed the decreased NPHS2 staining in aging kidneys. Scalar bar: $50 \mu \mathrm{m}$. N=5-6 for each group. C) Kidney injury markers TGF- $\beta$, PAI-1, Col4a1, F4/80, and NGAL mRNA expression were increased in aging kidneys but decreased with ERR treatment. $\mathrm{N}=5-6$ for each group. D) Cytokine array showed 4 major spots that correspond to kidney injury markers Ngal, Kim1, Osteopontin and Ccl21, with increased expression in the aging kidney and decreased expression following the pan agonist treatment. Each group was one sample pooled from $n=4$ animals.

Figure 4. RNAseq and proteomics of kidney from old mice treated with vehicle or the pan-ERR agonist.

A) ERRa, ERR $\beta$ and ERR $\gamma$ mRNA expression were decreased in aging mouse kidneys. Treatment with the pan ERR agonist restored the mRNA levels of ERRa, ERR $\beta$ and ERR $\gamma$ to levels seen in young kidneys. $\mathrm{N}=5-6$ for each group. B) Heat map showing expression patterns of genes differentially expressed in kidneys of old mice treated with 
vehicle compared to kidneys of old mice treated with pan-ERR agonist. The heat map indicates up-regulation (green), down-regulation (red), and unaltered gene expression (black). The columns represent individual samples. C) Functional pathway enrichment analysis of differentially expressed proteins in kidneys of old mice treated with vehicle compared to kidneys of old mice treated with pan ERR agonist. The y-axis shows significantly enriched pathways. The x-axis indicates $p$-value of enrichment of the given pathway. D) Heat map showing expression patterns of proteins differentially expressed in kidneys of old mice treated with vehicle compared to kidneys of old mice treated with pan ERR agonist. The heat map indicates up-regulation (green), down-regulation (red), and unaltered gene expression (black). The columns represent individual samples. E) Functional pathway enrichment analysis of differentially expressed proteins in old mice treated with vehicle compared to kidneys of old mice treated with pan ERR agonist. The $y$-axis shows significantly enriched pathways. The $x$-axis indicates $p$-value of enrichment of the given pathway. F) Functional pathway enrichment analysis of subset of genes and proteins identified with O2PLS analysis as upregulated in kidneys of old mice compared to kidneys of young mice and that were downregulated by ERR treatment in old mice. The $\mathrm{y}$-axis shows significantly enriched pathways. The $\mathrm{x}$-axis indicates p-value of enrichment of the given pathway. G) Functional pathway enrichment analysis of subset of genes and proteins identified with O2PLS analysis as downregulated in kidneys of old mice compared to kidneys of young mice and that were upregulated by ERR treatment in old mice. The y-axis shows significantly enriched pathways. The x-axis indicates $p$-value of enrichment of the given pathway. 
Figure 5. Pan-ERR agonist treatment restored mitochondrial function in aging

\section{kidneys.}

A) Pan-ERR agonist treatment in old mice increased the expression of PCG1a and PGC1 $\beta$, coregulators of ERRs and mediators of mitochondrial biogenesis. The expression of the mitochondrial transcription factor Tfam1 was decreased in the kidneys of old mice and pan ERR agonist treatment restored it to levels seen in young mice. Mitochondria to nuclear DNA ratio was decreased in the kidneys of old mice and pan ERR agonist treatment restored it to levels seen in young mice, indicative of increased mitochondrial biogenesis. $\mathrm{N}=5-6$ for each group. B) mRNA expression levels of subunits for mitochondrial electron chain complex (ETC) was decreased in the kidneys of old mice and pan ERR agonist treatment restored it to levels seen in young mice, indicative of improvement in ETC. $\mathrm{N}=5-6$ for each group. C) mRNA expression levels of the TCA cycle enzymes were decreased in the kidneys of old mice and recovered following pan-ERR agonist treatment to levels seen in young mice, indicative of restoration of the TCA cycle. One of the TCA cycle intermediates, succinic acid showed increased level in the kidneys of old mice treated with the pan ERR agonist. N=5-6 for each group. D) Autofluorescence intensity (NADH channel) (top) and Phasor mapped FLIM image (bottom) on the kidney section. Scale bar $=100 \mu \mathrm{m}$. The phasor mapped FLIM images were pseudo colored based on the fractional intensity of the free NADH as shown in the color scale. The fractional intensity contributions were calculated by resolving the phasor signatures from these images in between the phasor positions of free and bound NADH. Fractional intensity of NADH plot showed that there was decreased free NADH fraction in aging kidneys and pan-ERR agonist treatment 
increased the free $\mathrm{NADH}$ fraction. $\mathrm{N}=5-6$ for each group. E) Interrelationship of the TCA cycle and ETC. F) Maximum respiration capacity in mitochondria isolated from the kidneys showed a significant impairment in old mice, which is restored to levels seen in young mice after treatment with the pan ERR agonist. N=5-6 for each group. G) The fatty acid $\beta$-oxidation enzymes Cpt1a and Mcad mRNA levels were decreased in the kidneys of old mice, which were restored to levels seen in young mice after treatment with the pan ERR agonist. $\mathrm{N}=5-6$ for each group.

\section{Figure 6. Pan-ERR agonist treatment altered mitochondrial dynamics in aging}

\section{Kidneys.}

A) Transmission electron microscopy showed alterations in the mitochondria of aging kidneys including decreases in the area, perimeter, and minimum Feret diameter, which were restored to levels seen in young kidneys upon treatment with the pan ERR agonist. $\mathrm{N}=3-4$ for each group. $\mathrm{B}$ ) There was a significant decrease in mitofusin-2 protein abundance in the old kidneys and the pan-ERR agonist increased the protein abundance in both the young and the old kidneys, with the resulting levels in the old kidneys being the same as in the young kidneys. In contrast, there was no significant change in the protein level of Opa1 in the old kidneys. However, upon treatment with the pan-ERR agonist, there was a tendency for the protein level to increase in the aging kidneys. There were also significant decreases in mitoguardin 2 and MitoPLD mRNA levels in the aging kidneys that were normalized upon treatment with the pan-ERR agonist. In addition, there were significant increases in Drp1 and phospho-Drp1 protein in the kidneys of old mice, which were restored back to levels seen in young mice 
following treatment with the pan ERR agonist. $\mathrm{N}=5-6$ for each group in mRNA level analysis. $\mathrm{N}=4$ for each group in protein analysis.

Figure 7. Pan-ERR agonist treatment decreased inflammation in aging kidneys. A) There were significant increases in STING and cGAS mRNA and protein level in the kidneys of old mice. Treatment with the pan ERR agonist decreased their expression in the kidneys of old mice. $\mathrm{N}=5-6$ for each group in mRNA level analysis. $\mathrm{N}=4$ for each group in protein analysis. B) RIG-I-like receptors (RIG-I, MDA5, LGP2) and Toll-like receptors (TLR3, 7 and 9) mRNA levels were increased in the aging kidney and decreased with the treatment. $\mathrm{N}=5-6$ for each group. C) There were significant increases in Rel, Relb and Nfkb2 mRNA, and total p65 protein expression in the kidneys of old mice and treatment with the pan ERR agonist decreased their expression to levels seen in the kidneys of young mice. There were also similar changes to Stat3 mRNA, and p-Tyr705-STAT3 and total STAT3 protein expression. $\mathrm{N}=5-6$ for each group in mRNA level analysis. $\mathrm{N}=3-4$ for each group in protein analysis. D) There were significant increases in the cellular senescence marker p21 and p16 in the aging kidney and the pan agonist treatment reduced the level of p21 expression but not p16. As senescence associated secretory phenotype marker, II1b, Icam1, Timp1, Tnfa, and Ccr5 mRNA were found increased in the kidneys of old mice and treatment with the pan ERR agonist decreased their expression. $\mathrm{N}=5-6$ for each group.

Figure 8. Treatment of aging mice with STING inhibitor C-176. 
A) STING inhibitor decreased IL-1 $\beta$, Stat3, phospho-Stat3, and p21 expression in aging

kidneys. $\mathrm{N}=4$ for each group. B) STING inhibitor increased expression of PGC1 $\alpha$,

PGC1 $\beta$, ERR $\alpha$, Ndufs1 (complex I), Cox6a2 (complex IV), Atp5e (complex V), and

MCAD in aging kidneys. $\mathrm{N}=4$ for each group. 


\section{ACKNOWLEDGEMENTS}

The above study is supported by NIA R01 AG049493 and NIDDK R01 DK116567 to M.L. and AHA postdoctoral fellowship to K.M. (19POST34381041) and A.E. L. (19POST34430001). J.B.K. is supported by the NIDDK Intramural Research Program. F.J.G and U.G. are supported by the NCI Intramural Research Program.

\section{AUTHOR CONTRIBUTIONS}

This study was conceived and led by M.L. and X.X.W. X.X.W. performed most of the laboratory work. K.M. assisted with the animal studies and biochemical analysis. Y.Q. and U.G. performed the proteomics and assisted with the analysis. J.P. A.T., V.S., B.O., and L.B. performed the multi-omics processing and integration analyses. S.R. and K.B. conducted the FLIM imaging and analysis. P.D. and A.Z.R. performed the immunohistochemistry and analysis. A.E.L. performed human primary cell culture work. P.Z. and J.B.K. performed the EM and B.A.J., K.B. and A.Z.R. assisted with the data analysis. T.B. provided the reagent pan-ERR agonist. X.X.W. and M.L. wrote the manuscript with editorial input from all authors.

\section{COMPETING INTERESTS}

The authors declare no competing interests.

\section{DATA AVAILABILITY STATEMENT}


The data that support the findings of this study are available from the corresponding author upon reasonable request. RNAseq raw data have been deposited in NCBI GEO database with accession number: PRJNA642362. Proteomics data are available via ProteomeXchange with identifier PXD020051. 


\section{REFERENCES}

1. Tonelli, M, Riella, M: Chronic kidney disease and the aging population. American journal of physiology Renal physiology, 306: F469-472, 2014.

2. Choudhury, D, Levi, M: Kidney aging--inevitable or preventable? Nature reviews Nephrology, 7: 706-717, 2011.

3. Hommos, MS, Glassock, RJ, Rule, AD: Structural and Functional Changes in Human Kidneys with Healthy Aging. Journal of the American Society of Nephrology : JASN, 28: 2838-2844, 2017.

4. Berg, UB: Differences in decline in GFR with age between males and females. Reference data on clearances of inulin and PAH in potential kidney donors. Nephrology, dialysis, transplantation : official publication of the European Dialysis and Transplant Association - European Renal Association, 21: 2577-2582, 2006.

5. Tauchi, H, Tsuboi, K, Okutomi, J: Age changes in the human kidney of the different races. Gerontologia, 17: 87-97, 1971.

6. Fliser, D, Franek, E, Joest, M, Block, S, Mutschler, E, Ritz, E: Renal function in the elderly: impact of hypertension and cardiac function. Kidney international, 51: 1196-1204, 1997.

7. Jang, JY, Blum, A, Liu, J, Finkel, T: The role of mitochondria in aging. J Clin Invest, 128: 3662-3670, 2018.

8. Shpilka, T, Haynes, CM: The mitochondrial UPR: mechanisms, physiological functions and implications in ageing. Nat Rev Mol Cell Biol, 19: 109-120, 2018.

9. Hansen, M, Rubinsztein, DC, Walker, DW: Autophagy as a promoter of longevity: insights from model organisms. Nat Rev Mol Cell Biol, 19: 579-593, 2018.

10. Jiang, T, Liebman, SE, Lucia, MS, Li, J, Levi, M: Role of altered renal lipid metabolism and the sterol regulatory element binding proteins in the pathogenesis of age-related renal disease. Kidney Int, 68: 2608-2620, 2005.

11. Jiang, T, Liebman, SE, Lucia, MS, Phillips, CL, Levi, M: Calorie restriction modulates renal expression of sterol regulatory element binding proteins, lipid accumulation, and age-related renal disease. J Am Soc Nephrol, 16: 2385-2394, 2005.

12. Wang, XX, Luo, Y, Wang, D, Adorini, L, Pruzanski, M, Dobrinskikh, E, Levi, M: A dual agonist of farnesoid $X$ receptor $(F X R)$ and the $G$ protein-coupled receptor TGR5, INT-767, reverses age-related kidney disease in mice. J Biol Chem, 292: 12018-12024, 2017.

13. Wei, W, Schwaid, AG, Wang, X, Wang, X, Chen, S, Chu, Q, Saghatelian, A, Wan, Y: Ligand Activation of ERRalpha by Cholesterol Mediates Statin and Bisphosphonate Effects. Cell Metab, 23: 479-491, 2016.

14. Audet-Walsh, E, Giguere, V: The multiple universes of estrogen-related receptor alpha and gamma in metabolic control and related diseases. Acta Pharmacol Sin, 36: 51-61, 2015.

15. Finck, BN, Kelly, DP: PGC-1 coactivators: inducible regulators of energy metabolism in health and disease. J Clin Invest, 116: 615-622, 2006.

16. Fritah, A, Christian, M, Parker, MG: The metabolic coregulator RIP140: an update. Am J Physiol Endocrinol Metab, 299: E335-340, 2010. 
17. Yamamoto, H, Williams, EG, Mouchiroud, L, Canto, C, Fan, W, Downes, M, Heligon, C, Barish, GD, Desvergne, B, Evans, RM, Schoonjans, K, Auwerx, J: NCoR1 is a conserved physiological modulator of muscle mass and oxidative function. Cell, 147: 827-839, 2011.

18. Tremblay, AM, Wilson, BJ, Yang, XJ, Giguere, V: Phosphorylation-dependent sumoylation regulates estrogen-related receptor-alpha and -gamma transcriptional activity through a synergy control motif. Mol Endocrinol, 22: 570584, 2008.

19. Wilson, BJ, Tremblay, AM, Deblois, G, Sylvain-Drolet, G, Giguere, V: An acetylation switch modulates the transcriptional activity of estrogen-related receptor alpha. Mol Endocrinol, 24: 1349-1358, 2010.

20. Ariazi, EA, Clark, GM, Mertz, JE: Estrogen-related receptor alpha and estrogenrelated receptor gamma associate with unfavorable and favorable biomarkers, respectively, in human breast cancer. Cancer Res, 62: 6510-6518, 2002.

21. Huss, JM, Garbacz, WG, Xie, W: Constitutive activities of estrogen-related receptors: Transcriptional regulation of metabolism by the ERR pathways in health and disease. Biochim Biophys Acta, 1852: 1912-1927, 2015.

22. Sonoda, J, Laganiere, J, Mehl, IR, Barish, GD, Chong, LW, Li, X, Scheffler, IE, Mock, DC, Bataille, AR, Robert, F, Lee, CH, Giguere, V, Evans, RM: Nuclear receptor ERR alpha and coactivator PGC-1 beta are effectors of IFN-gammainduced host defense. Genes Dev, 21: 1909-1920, 2007.

23. Bookout, AL, Jeong, Y, Downes, M, Yu, RT, Evans, RM, Mangelsdorf, DJ: Anatomical profiling of nuclear receptor expression reveals a hierarchical transcriptional network. Cell, 126: 789-799, 2006.

24. Berry, R, Harewood, L, Pei, L, Fisher, M, Brownstein, D, Ross, A, Alaynick, WA, Moss, J, Hastie, ND, Hohenstein, P, Davies, JA, Evans, RM, FitzPatrick, DR: Esrrg functions in early branch generation of the ureteric bud and is essential for normal development of the renal papilla. Hum Mol Genet, 20: 917-926, 2011.

25. Wang, XX, Edelstein, MH, Gafter, U, Qiu, L, Luo, Y, Dobrinskikh, E, Lucia, S, Adorini, L, D'Agati, VD, Levi, J, Rosenberg, A, Kopp, JB, Gius, DR, Saleem, MA, Levi, M: G Protein-Coupled Bile Acid Receptor TGR5 Activation Inhibits Kidney Disease in Obesity and Diabetes. J Am Soc Nephrol, 27: 1362-1378, 2016.

26. Huang da, W, Sherman, BT, Lempicki, RA: Systematic and integrative analysis of large gene lists using DAVID bioinformatics resources. Nat Protoc, 4: 44-57, 2009.

27. Mi, H, Muruganujan, A, Ebert, D, Huang, X, Thomas, PD: PANTHER version 14: more genomes, a new PANTHER GO-slim and improvements in enrichment analysis tools. Nucleic Acids Res, 47: D419-D426, 2019.

28. Babicki, S, Arndt, D, Marcu, A, Liang, Y, Grant, JR, Maciejewski, A, Wishart, DS: Heatmapper: web-enabled heat mapping for all. Nucleic Acids Res, 44: W147153, 2016.

29. Park, J, Shrestha, R, Qiu, C, Kondo, A, Huang, S, Werth, M, Li, M, Barasch, J, Susztak, K: Single-cell transcriptomics of the mouse kidney reveals potential cellular targets of kidney disease. Science, 360: 758-763, 2018. 
30. Wu, H, Kirita, Y, Donnelly, EL, Humphreys, BD: Advantages of Single-Nucleus over Single-Cell RNA Sequencing of Adult Kidney: Rare Cell Types and Novel Cell States Revealed in Fibrosis. J Am Soc Nephrol, 30: 23-32, 2019.

31. Zhang, X, Nguyen, KD, Rudnick, PA, Roper, N, Kawaler, E, Maity, TK, Awasthi, S, Gao, S, Biswas, R, Venugopalan, A, Cultraro, CM, Fenyo, D, Guha, U: Quantitative Mass Spectrometry to Interrogate Proteomic Heterogeneity in Metastatic Lung Adenocarcinoma and Validate a Novel Somatic Mutation CDK12-G879V. Mol Cell Proteomics, 18: 622-641, 2019.

32. Cox, J, Neuhauser, N, Michalski, A, Scheltema, RA, Olsen, JV, Mann, M:

Andromeda: a peptide search engine integrated into the MaxQuant environment. J Proteome Res, 10: 1794-1805, 2011.

33. Zhang, Y, Jenkins, DF, Manimaran, S, Johnson, WE: Alternative empirical Bayes models for adjusting for batch effects in genomic studies. BMC Bioinformatics, 19: 262, 2018.

34. Casella, G, Berger, RL: Statistical inference, Australia ; Pacific Grove, CA, Thomson Learning, 2002.

35. Bouhaddani, SE, Uh, HW, Jongbloed, G, Hayward, C, Klaric, L, Kielbasa, SM, Houwing-Duistermaat, J: Integrating omics datasets with the OmicsPLS package. BMC Bioinformatics, 19: 371, 2018.

36. Jiang, T, Wang, XX, Scherzer, P, Wilson, P, Tallman, J, Takahashi, H, Li, J, Iwahashi, M, Sutherland, E, Arend, L, Levi, M: Farnesoid X receptor modulates renal lipid metabolism, fibrosis, and diabetic nephropathy. Diabetes, 56: 24852493, 2007.

37. Wang, XX, Jiang, T, Shen, Y, Caldas, Y, Miyazaki-Anzai, S, Santamaria, H, Urbanek, C, Solis, N, Scherzer, P, Lewis, L, Gonzalez, FJ, Adorini, L, Pruzanski, M, Kopp, JB, Verlander, JW, Levi, M: Diabetic nephropathy is accelerated by farnesoid $X$ receptor deficiency and inhibited by farnesoid $X$ receptor activation in a type 1 diabetes model. Diabetes, 59: 2916-2927, 2010.

38. Wang, XX, Jiang, T, Shen, Y, Adorini, L, Pruzanski, M, Gonzalez, FJ, Scherzer, P, Lewis, L, Miyazaki-Anzai, S, Levi, M: The farnesoid $X$ receptor modulates renal lipid metabolism and diet-induced renal inflammation, fibrosis, and proteinuria. Am J Physiol Renal Physiol, 297: F1587-1596, 2009.

39. O'Sullivan, ED, Hughes, J, Ferenbach, DA: Renal Aging: Causes and Consequences. J Am Soc Nephrol, 28: 407-420, 2017.

40. Rayego-Mateos, S, Rodrigues-Diez, R, Morgado-Pascual, JL, Valentijn, F, Valdivielso, JM, Goldschmeding, R, Ruiz-Ortega, M: Role of Epidermal Growth Factor Receptor (EGFR) and Its Ligands in Kidney Inflammation and Damage. Mediators Inflamm, 2018: 8739473, 2018.

41. Yang, H, Fogo, AB: Cell senescence in the aging kidney. J Am Soc Nephrol, 21: 1436-1439, 2010.

42. Jankauskas, SS, Silachev, DN, Andrianova, NV, Pevzner, IB, Zorova, LD, Popkov, VA, Plotnikov, EY, Zorov, DB: Aged kidney: can we protect it? Autophagy, mitochondria and mechanisms of ischemic preconditioning. Cell Cycle, 17: 12911309, 2018.

43. Maekawa, H, Inoue, $T$, Ouchi, $H$, Jao, TM, Inoue, $R$, Nishi, $H$, Fujii, $R$, Ishidate, $F$, Tanaka, T, Tanaka, Y, Hirokawa, N, Nangaku, M, Inagi, R: Mitochondrial 
Damage Causes Inflammation via cGAS-STING Signaling in Acute Kidney Injury. Cell Rep, 29: 1261-1273 e1266, 2019.

44. Chung, KW, Dhillon, P, Huang, S, Sheng, X, Shrestha, R, Qiu, C, Kaufman, BA, Park, J, Pei, L, Baur, J, Palmer, M, Susztak, K: Mitochondrial Damage and Activation of the STING Pathway Lead to Renal Inflammation and Fibrosis. Cell Metab, 30: 784-799 e785, 2019.

45. Scarpulla, RC: Transcriptional paradigms in mammalian mitochondrial biogenesis and function. Physiol Rev, 88: 611-638, 2008.

46. Campbell, CT, Kolesar, JE, Kaufman, BA: Mitochondrial transcription factor A regulates mitochondrial transcription initiation, DNA packaging, and genome copy number. Biochim Biophys Acta, 1819: 921-929, 2012.

47. de Sousa Abreu, R, Penalva, LO, Marcotte, EM, Vogel, C: Global signatures of protein and mRNA expression levels. Mol Biosyst, 5: 1512-1526, 2009.

48. Nie, L, Wu, G, Culley, DE, Scholten, JC, Zhang, W: Integrative analysis of transcriptomic and proteomic data: challenges, solutions and applications. Crit Rev Biotechnol, 27: 63-75, 2007.

49. Leon-Mimila, P, Wang, J, Huertas-Vazquez, A: Relevance of Multi-Omics Studies in Cardiovascular Diseases. Front Cardiovasc Med, 6: 91, 2019.

50. Yan, J, Risacher, SL, Shen, L, Saykin, AJ: Network approaches to systems biology analysis of complex disease: integrative methods for multi-omics data. Brief Bioinform, 19: 1370-1381, 2018.

51. Bouhaddani, SE, Houwing-Duistermaat, J, Salo, P, Perola, M, Jongbloed, G, Uh, HW: Evaluation of O2PLS in Omics data integration. BMC Bioinformatics, 17 Suppl 2: 11, 2016.

52. Haas, RH: Mitochondrial Dysfunction in Aging and Diseases of Aging. Biology (Basel), 8, 2019.

53. Sato, Y, Yanagita, M: Immunology of the ageing kidney. Nature Reviews Nephrology, 2019.

54. Ranjit, S, Malacrida, L, Stakic, M, Gratton, E: Determination of the metabolic index using the fluorescence lifetime of free and bound nicotinamide adenine dinucleotide using the phasor approach. J Biophotonics, 12: e201900156, 2019.

55. Ranjit, S, Malacrida, L, Jameson, DM, Gratton, E: Fit-free analysis of fluorescence lifetime imaging data using the phasor approach. Nat Protoc, 13: 1979-2004, 2018.

56. Yaseen, MA, Sutin, J, Wu, W, Fu, B, Uhlirova, H, Devor, A, Boas, DA, Sakadzic, S: Fluorescence lifetime microscopy of NADH distinguishes alterations in cerebral metabolism in vivo. Biomed Opt Express, 8: 2368-2385, 2017.

57. Aguilar-Arnal, L, Ranjit, S, Stringari, C, Orozco-Solis, R, Gratton, E, Sassone-Corsi, P: Spatial dynamics of SIRT1 and the subnuclear distribution of NADH species. Proc Natl Acad Sci U S A, 113: 12715-12720, 2016.

58. Pernas, L, Scorrano, L: Mito-Morphosis: Mitochondrial Fusion, Fission, and Cristae Remodeling as Key Mediators of Cellular Function. Annu Rev Physiol, 78: 505531, 2016.

59. Liu, R, Chan, DC: OPA1 and cardiolipin team up for mitochondrial fusion. Nat Cell Biol, 19: 760-762, 2017. 
60. Zhang, Y, Liu, X, Bai, J, Tian, X, Zhao, X, Liu, W, Duan, X, Shang, W, Fan, HY, Tong, C: Mitoguardin Regulates Mitochondrial Fusion through MitoPLD and Is Required for Neuronal Homeostasis. Mol Cell, 61: 111-124, 2016.

61. Rodriguez-Nuevo, A, Zorzano, A: The sensing of mitochondrial DAMPs by nonimmune cells. Cell Stress, 3: 195-207, 2019.

62. Dhir, A, Dhir, S, Borowski, LS, Jimenez, L, Teitell, M, Rotig, A, Crow, YJ, Rice, GI, Duffy, D, Tamby, C, Nojima, T, Munnich, A, Schiff, M, de Almeida, CR, Rehwinkel, J, Dziembowski, A, Szczesny, RJ, Proudfoot, NJ: Mitochondrial double-stranded RNA triggers antiviral signalling in humans. Nature, 560: 238242, 2018.

63. Li, T, Chen, ZJ: The cGAS-cGAMP-STING pathway connects DNA damage to inflammation, senescence, and cancer. J Exp Med, 215: 1287-1299, 2018.

64. Majer, O, Liu, B, Barton, GM: Nucleic acid-sensing TLRs: trafficking and regulation. Curr Opin Immunol, 44: 26-33, 2017.

65. Rehwinkel, J, Gack, MU: RIG-I-like receptors: their regulation and roles in RNA sensing. Nat Rev Immunol, 2020.

66. Haag, SM, Gulen, MF, Reymond, L, Gibelin, A, Abrami, L, Decout, A, Heymann, M, van der Goot, FG, Turcatti, G, Behrendt, R, Ablasser, A: Targeting STING with covalent small-molecule inhibitors. Nature, 559: 269-273, 2018.

67. Madeo, F, Carmona-Gutierrez, D, Hofer, SJ, Kroemer, G: Caloric Restriction Mimetics against Age-Associated Disease: Targets, Mechanisms, and Therapeutic Potential. Cell Metab, 29: 592-610, 2019.

68. Lopez-Lluch, G, Navas, P: Calorie restriction as an intervention in ageing. J Physiol, 594: 2043-2060, 2016.

69. Sturmlechner, I, Durik, M, Sieben, CJ, Baker, DJ, van Deursen, JM: Cellular senescence in renal ageing and disease. Nat Rev Nephrol, 13: 77-89, 2017.

70. Fontana, L, Nehme, J, Demaria, M: Caloric restriction and cellular senescence. Mech Ageing Dev, 176: 19-23, 2018.

71. Ferrucci, L, Fabbri, E: Inflammageing: chronic inflammation in ageing, cardiovascular disease, and frailty. Nat Rev Cardiol, 15: 505-522, 2018.

72. Bang, E, Lee, B, Noh, SG, Kim, DH, Jung, HJ, Ha, S, Yu, BP, Chung, HY: Modulation of senoinflammation by calorie restriction based on biochemical and Omics big data analysis. BMB Rep, 52: 56-63, 2019.

73. Ablasser, A, Chen, ZJ: cGAS in action: Expanding roles in immunity and inflammation. Science, 363, 2019.

74. Ng, KW, Marshall, EA, Bell, JC, Lam, WL: cGAS-STING and Cancer: Dichotomous Roles in Tumor Immunity and Development. Trends Immunol, 39: 44-54, 2018.

75. Hsia, HC, Hutti, JE, Baldwin, AS: Cytosolic DNA Promotes Signal Transducer and Activator of Transcription 3 (STAT3) Phosphorylation by TANK-binding Kinase 1 (TBK1) to Restrain STAT3 Activity. J Biol Chem, 292: 5405-5417, 2017.

76. Kojima, H, Inoue, T, Kunimoto, H, Nakajima, K: IL-6-STAT3 signaling and premature senescence. JAKSTAT, 2: e25763, 2013.

77. Bienaime, F, Muorah, M, Yammine, L, Burtin, M, Nguyen, C, Baron, W, Garbay, S, Viau, A, Broueilh, M, Blanc, T, Peters, D, Poli, V, Anglicheau, D, Friedlander, G, Pontoglio, M, Gallazzini, M, Terzi, F: Stat3 Controls Tubulointerstitial Communication during CKD. J Am Soc Nephrol, 27: 3690-3705, 2016. 
78. Plotnikov, EY, Pevzner, IB, Zorova, LD, Chernikov, VP, Prusov, AN, Kireev, II, Silachev, DN, Skulachev, VP, Zorov, DB: Mitochondrial Damage and Mitochondria-Targeted Antioxidant Protection in LPS-Induced Acute Kidney Injury. Antioxidants (Basel), 8, 2019.

79. Tran, M, Tam, D, Bardia, A, Bhasin, M, Rowe, GC, Kher, A, Zsengeller, ZK, Akhavan-Sharif, MR, Khankin, EV, Saintgeniez, M, David, S, Burstein, D, Karumanchi, SA, Stillman, IE, Arany, Z, Parikh, SM: PGC-1alpha promotes recovery after acute kidney injury during systemic inflammation in mice. $J$ Clin Invest, 121: 4003-4014, 2011.

80. Cunningham, PN, Wang, Y, Guo, R, He, G, Quigg, RJ: Role of Toll-like receptor 4 in endotoxin-induced acute renal failure. J Immunol, 172: 2629-2635, 2004. 


\section{Supplementary Materials Table of Contents}

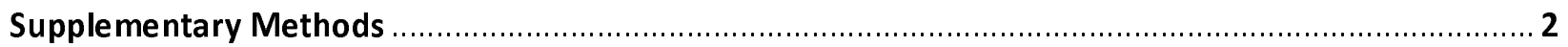

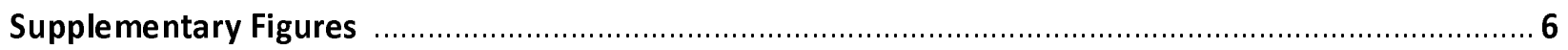

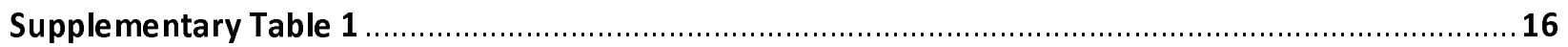

For supplementary tables 2-7 please refer to additional supporting documents (Supplementary tables 27.rar). 
Figure 1

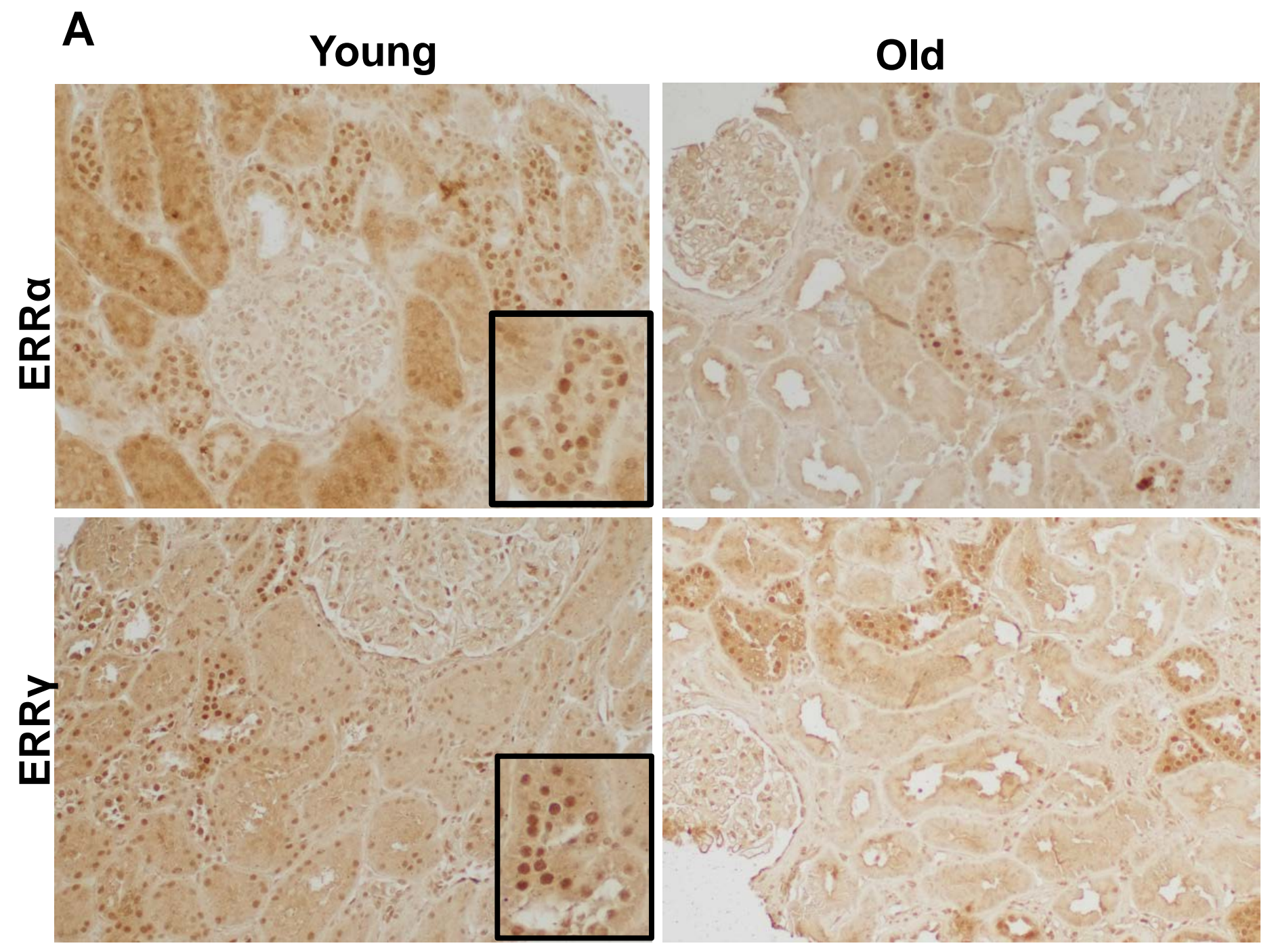

\section{B Young PDH}

Old PDH

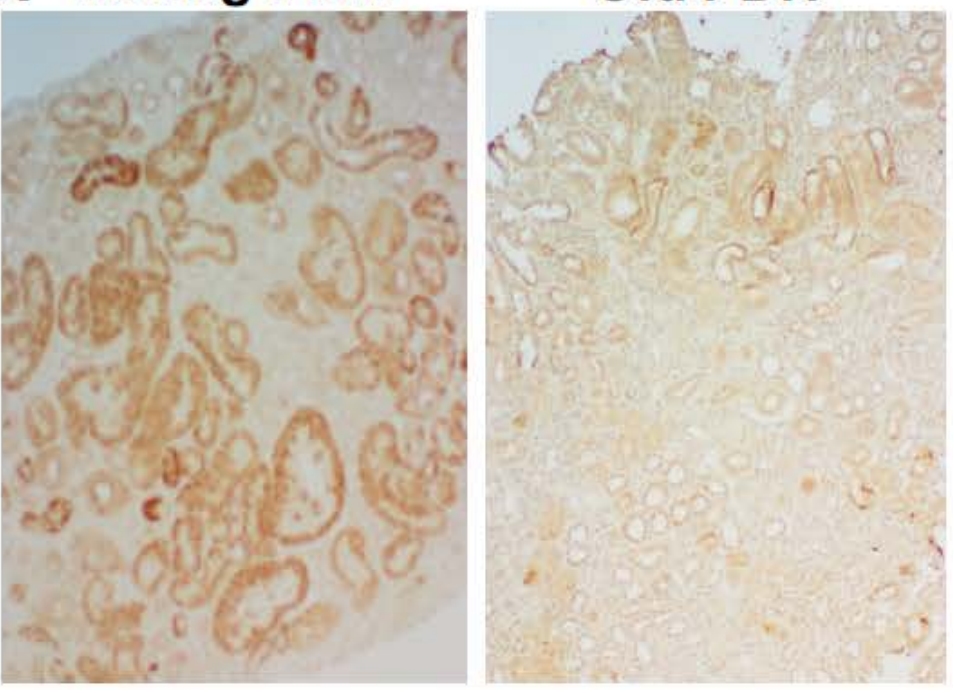


Figure 1

$$
\text { C }
$$
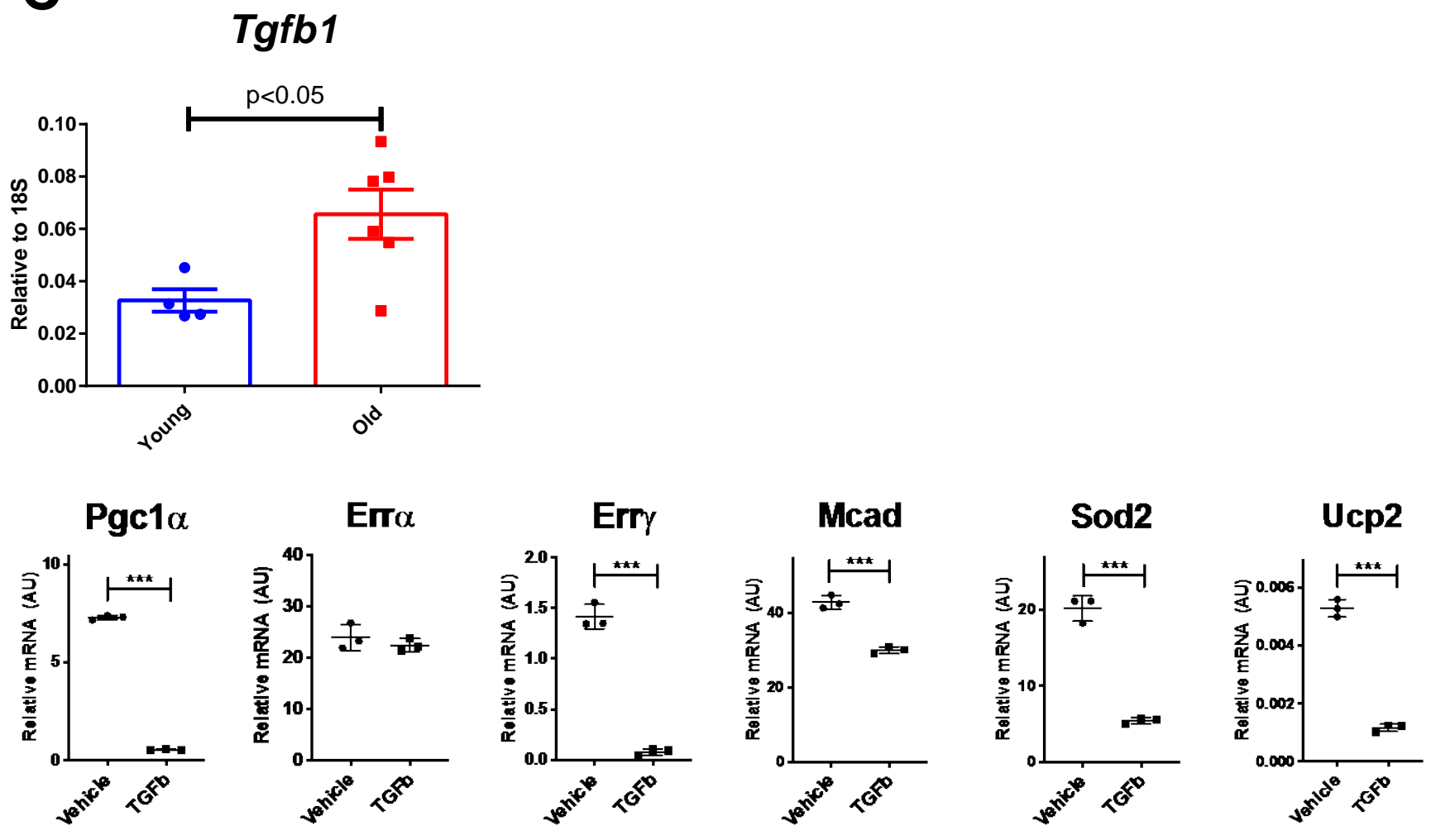

D
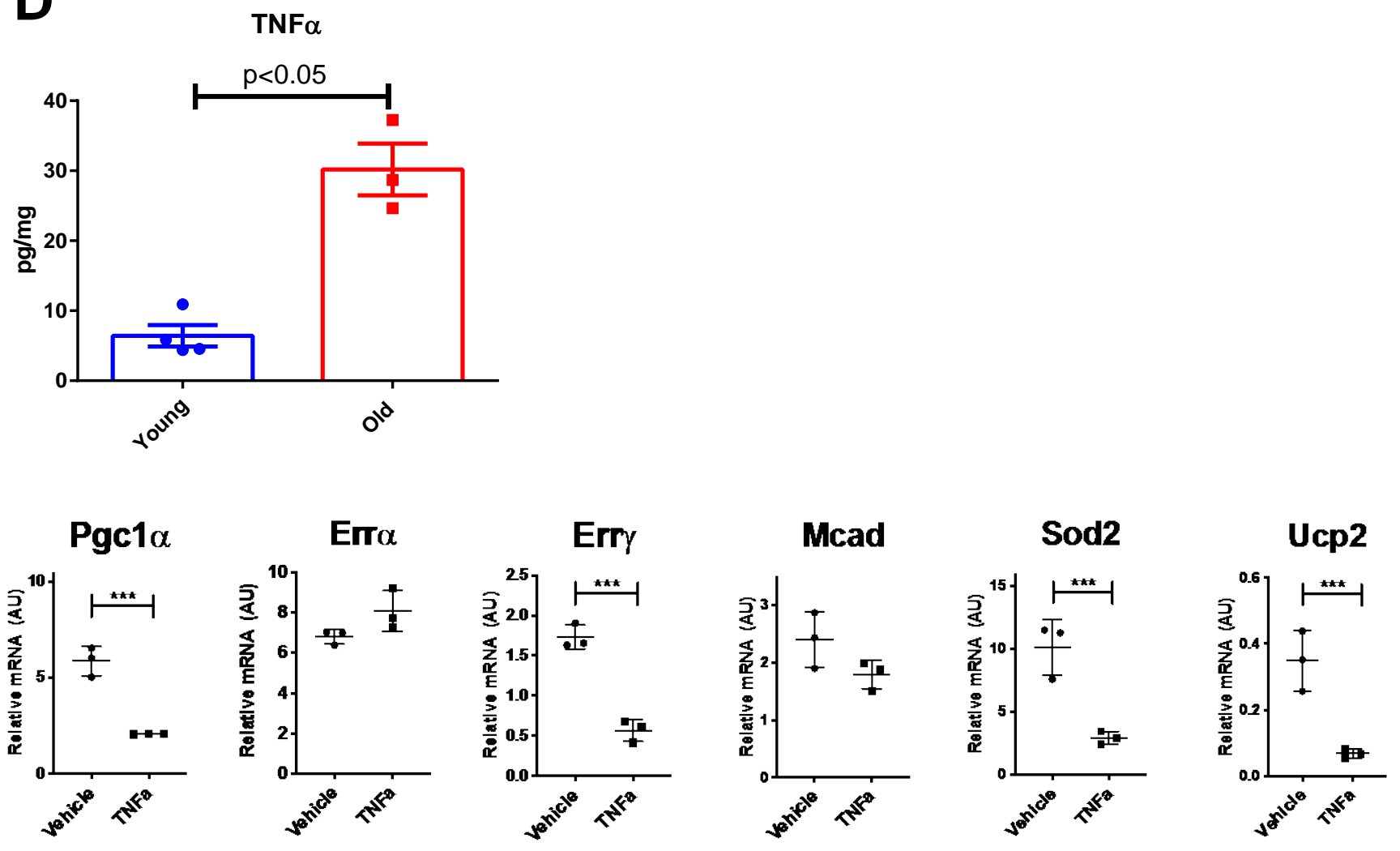
Figure 2

A

Young

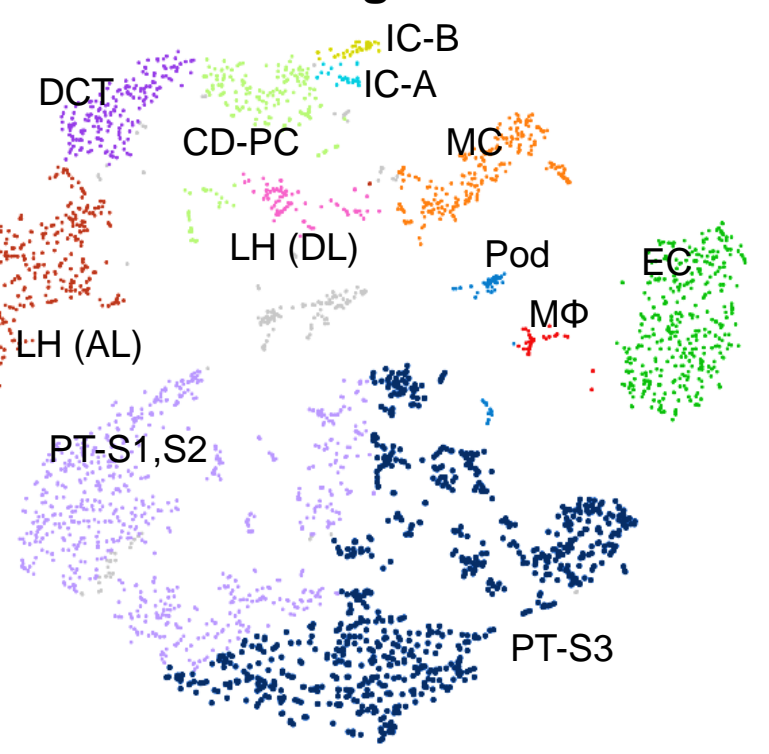

Old

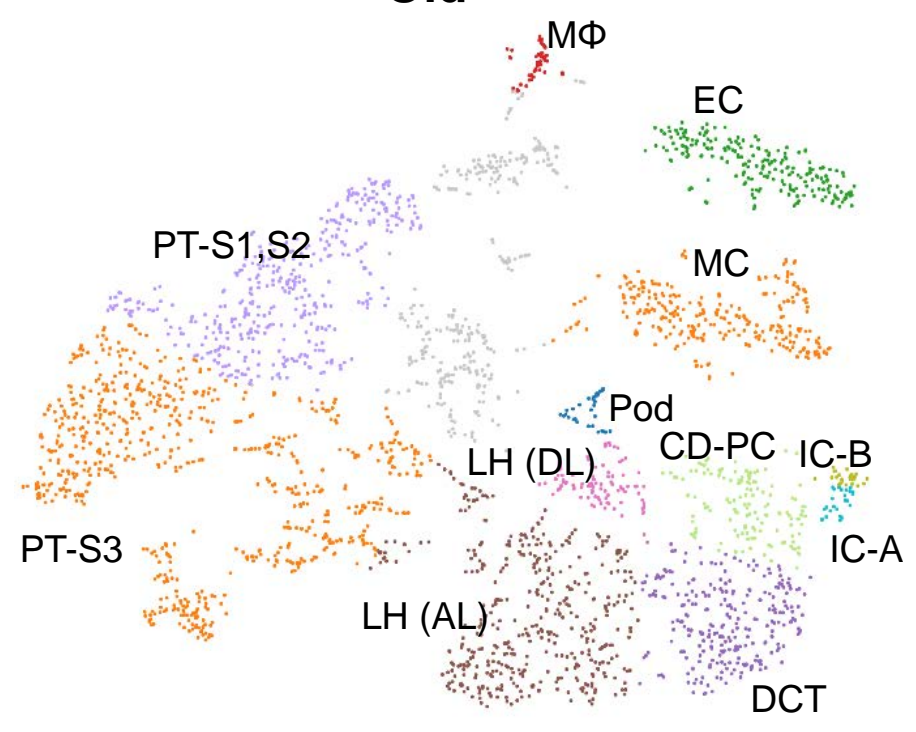

B

\section{Young}

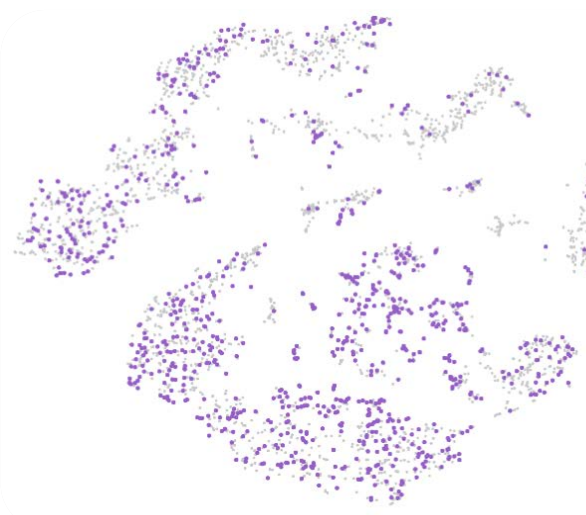

$22 \%$ cell count

\section{ERRa}

\section{Old}

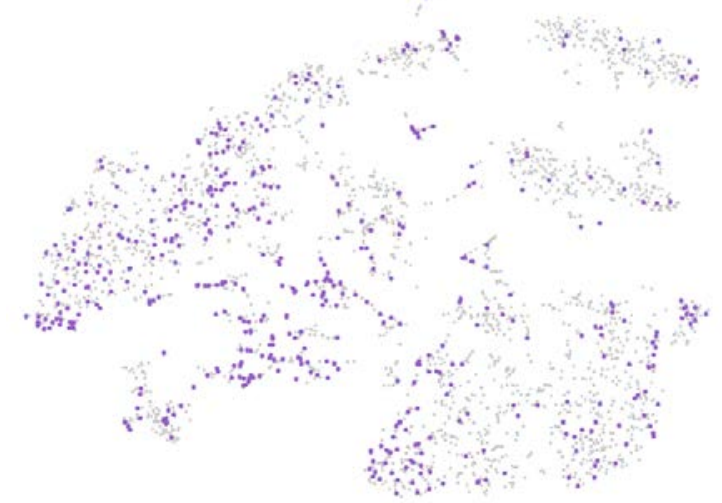

$17 \%$ cell count

\section{ERRY}


Figure 2

C

$\%$ of ERR $\alpha$ positive cells in each cluster

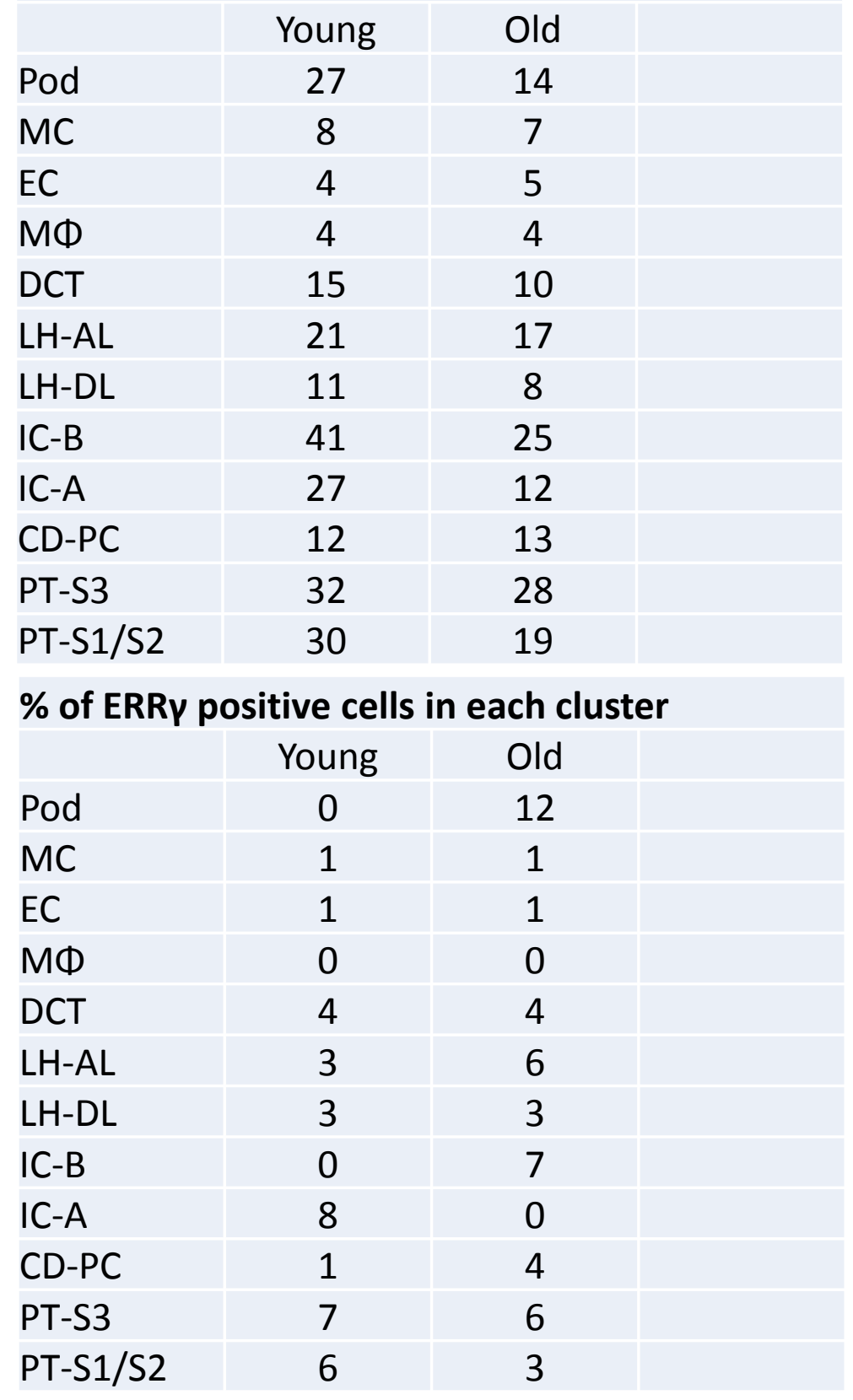




\section{Figure 3}

A

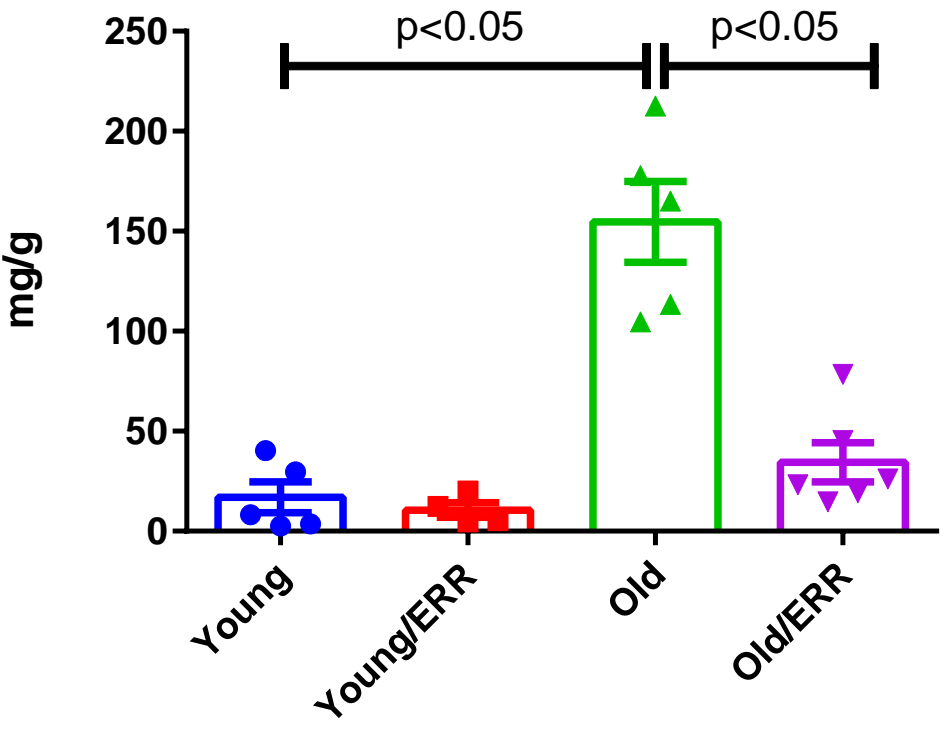

Kidney weight/Body weight

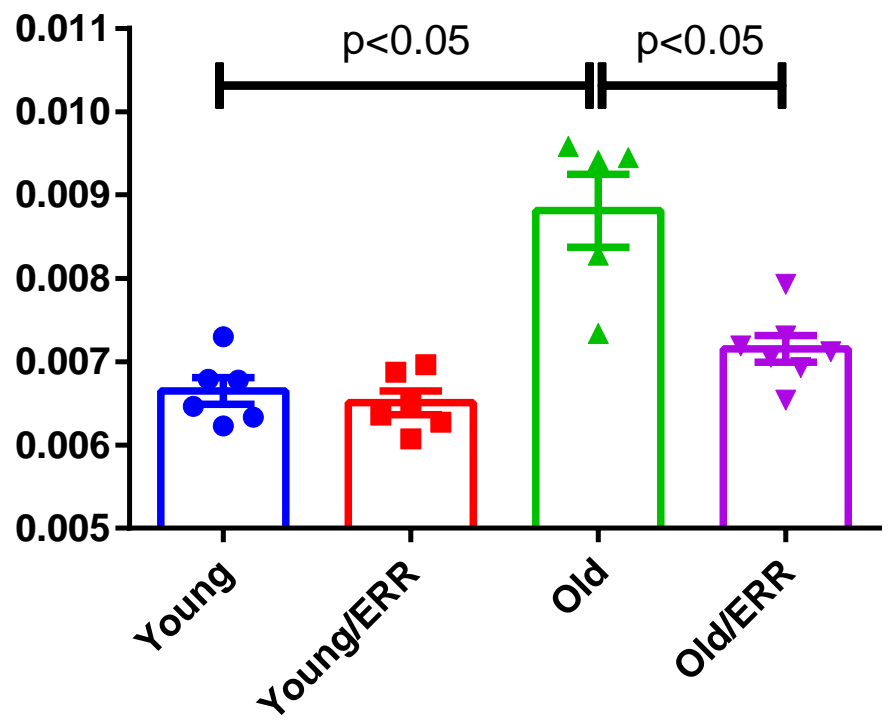

B
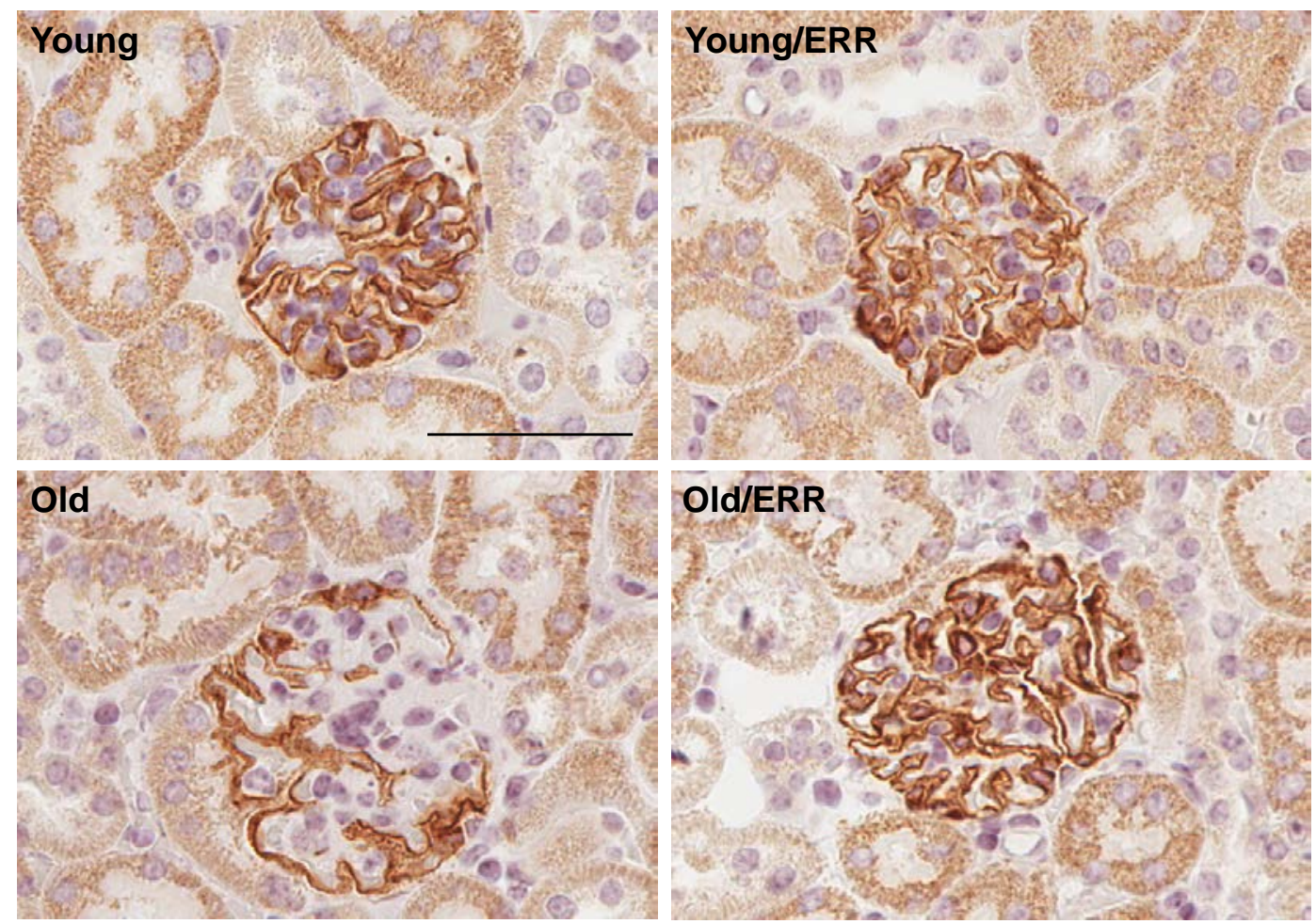

NPHS2 quantification

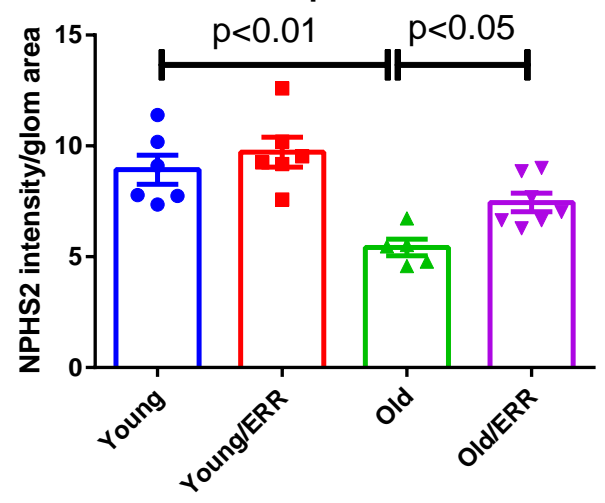


Figure 3

C

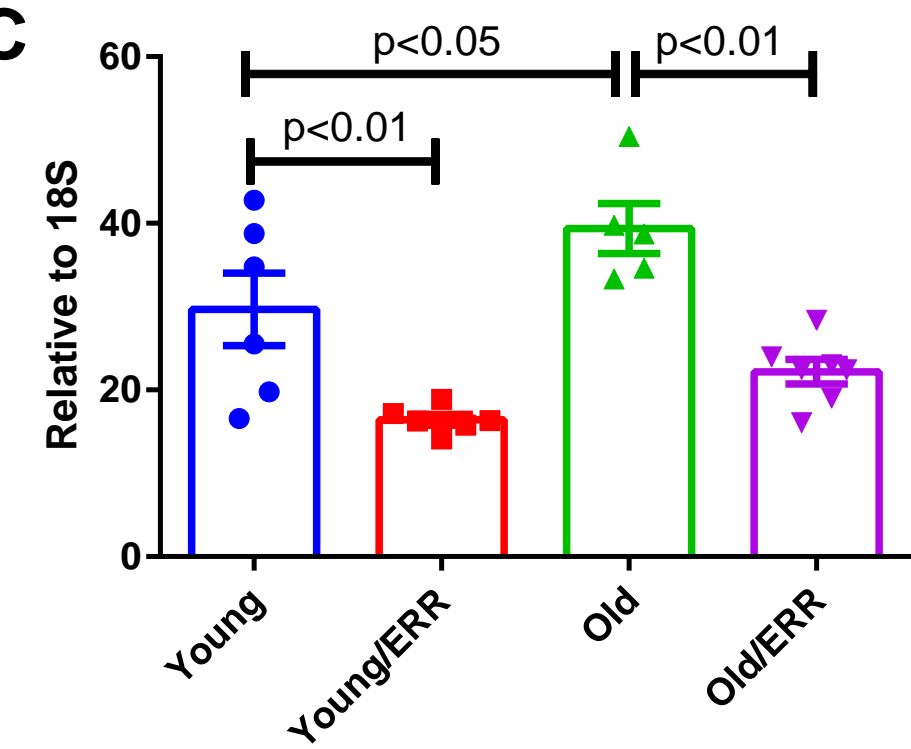

Col4a1 mRNA

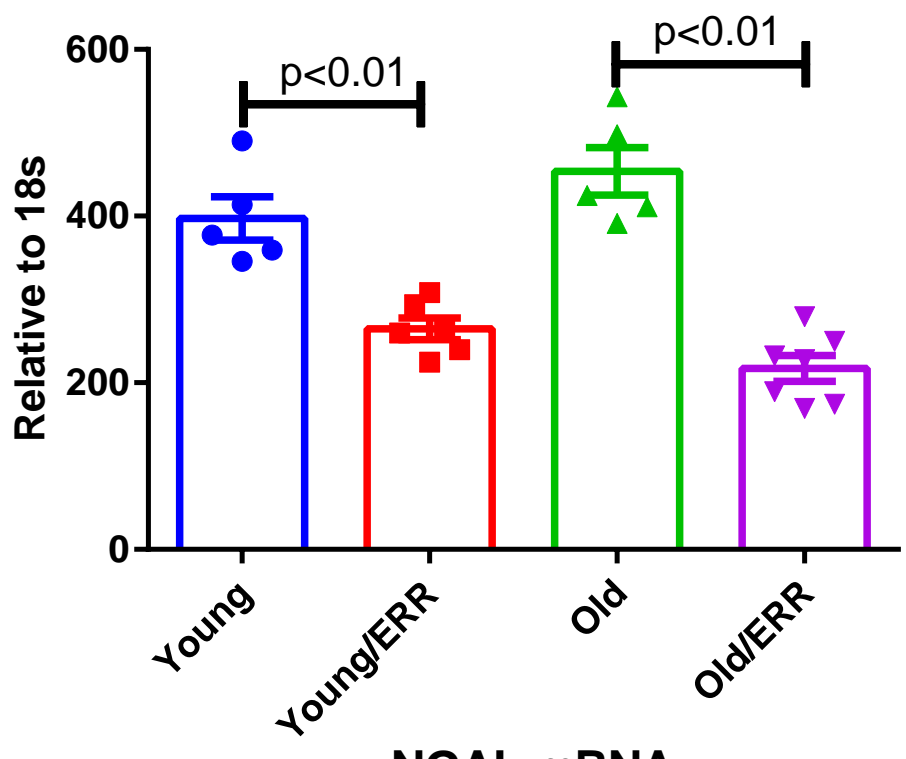

NGAL MRNA

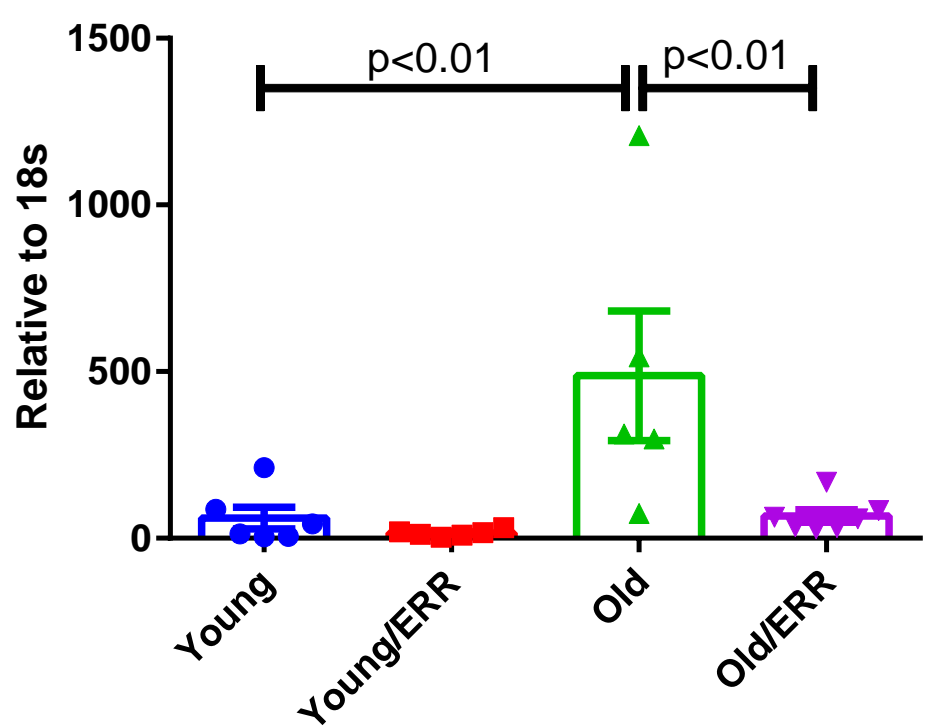

PAI-1 mRNA

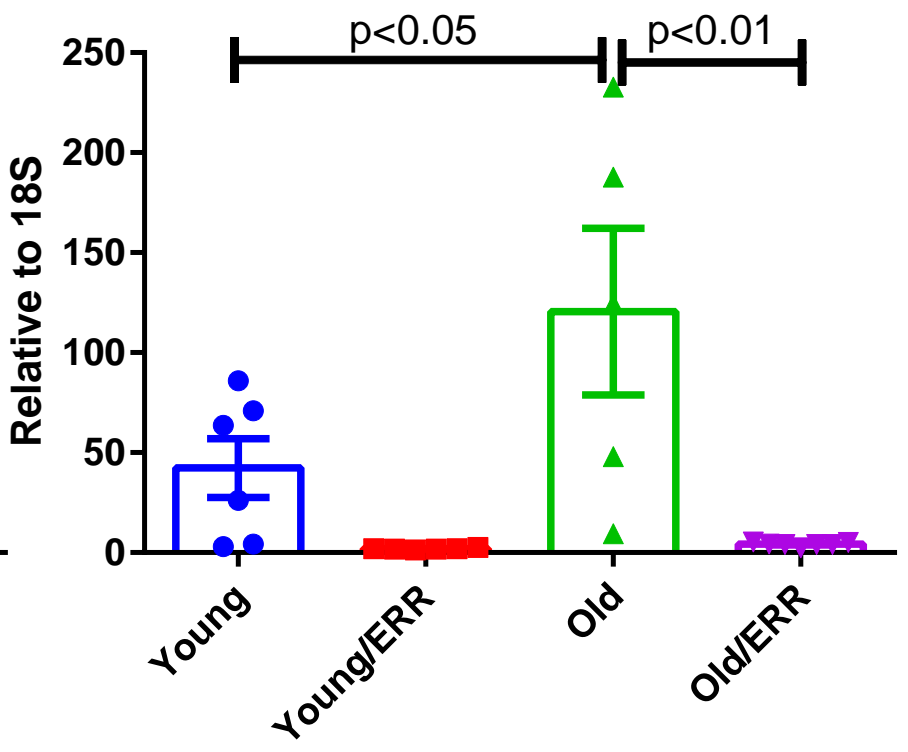

F4/80 mRNA

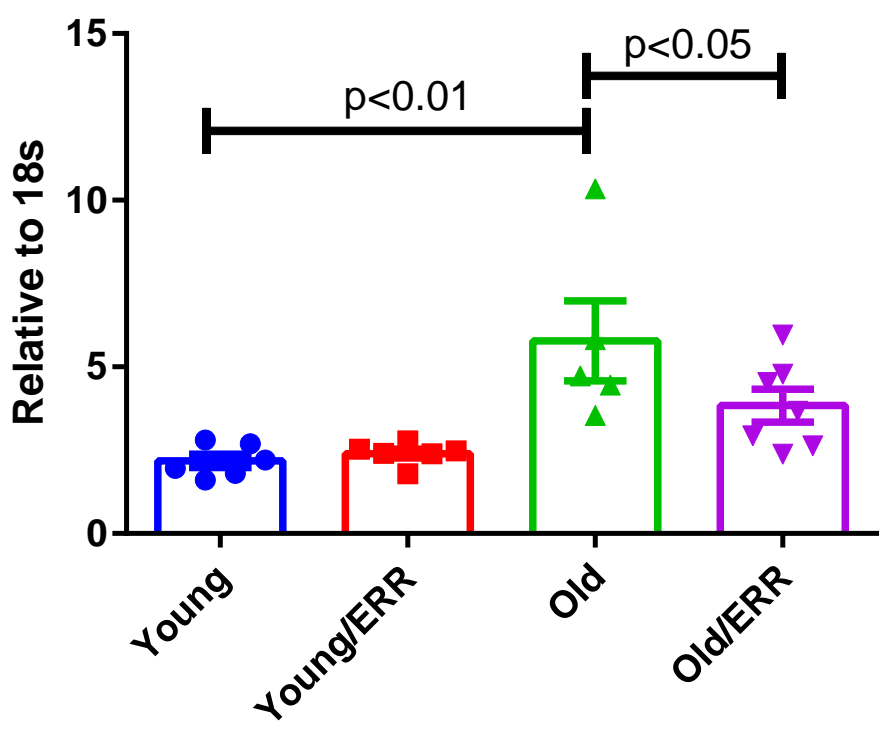



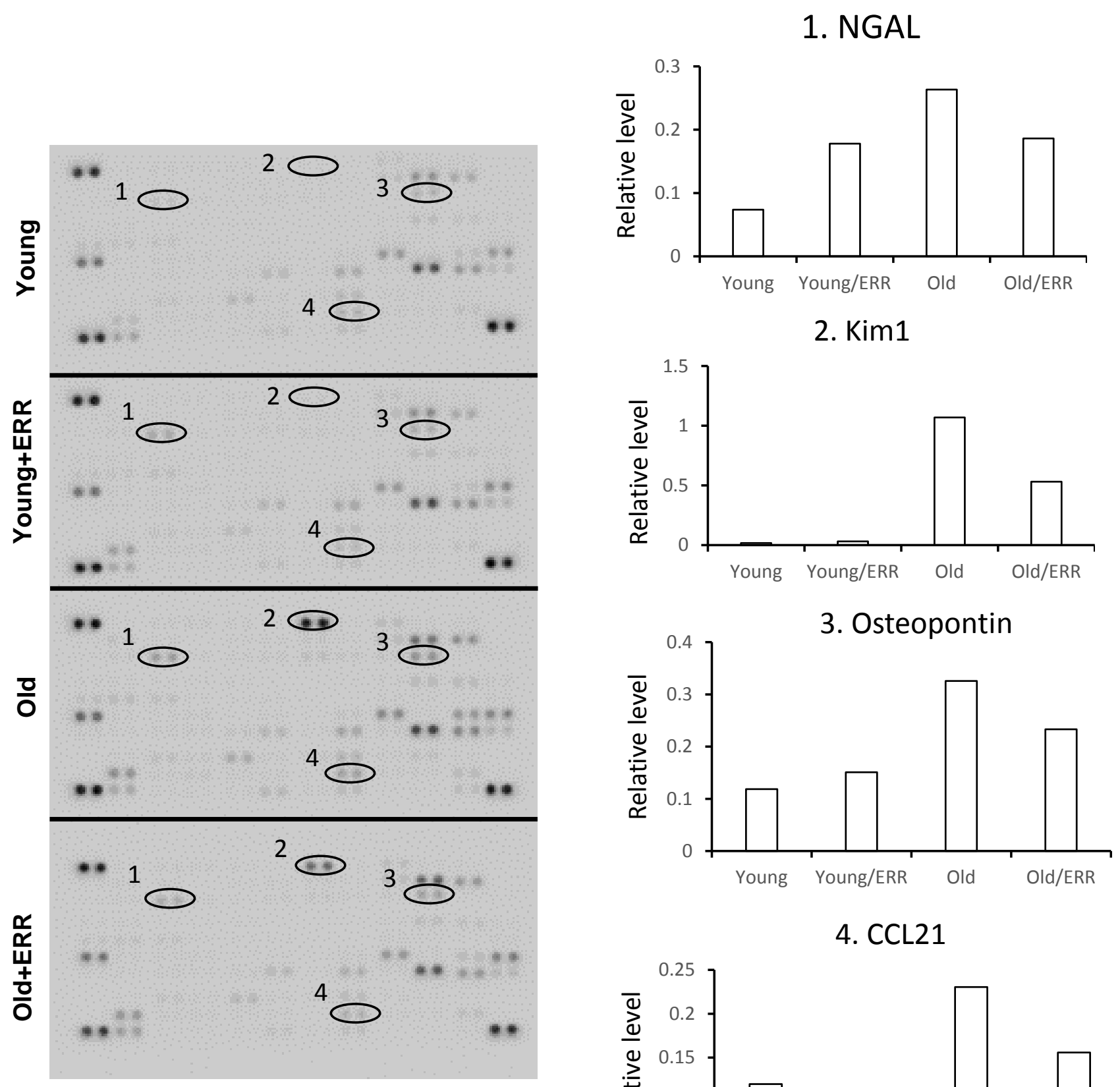

4. CCL21

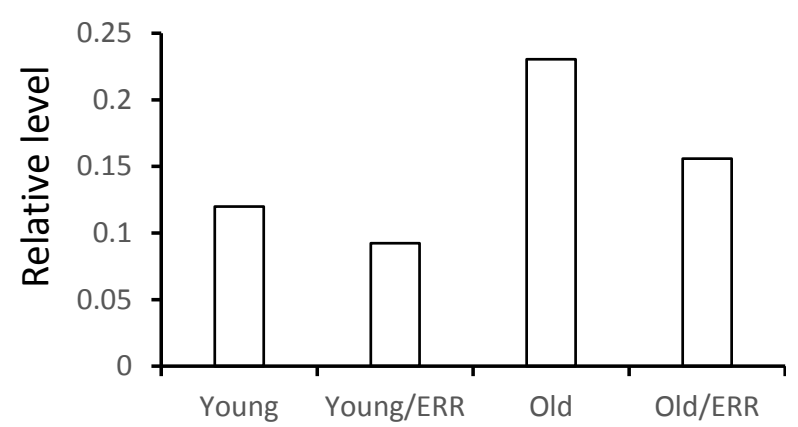


Figure 4

A
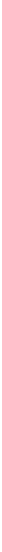


\section{Figure 4}

\section{B}

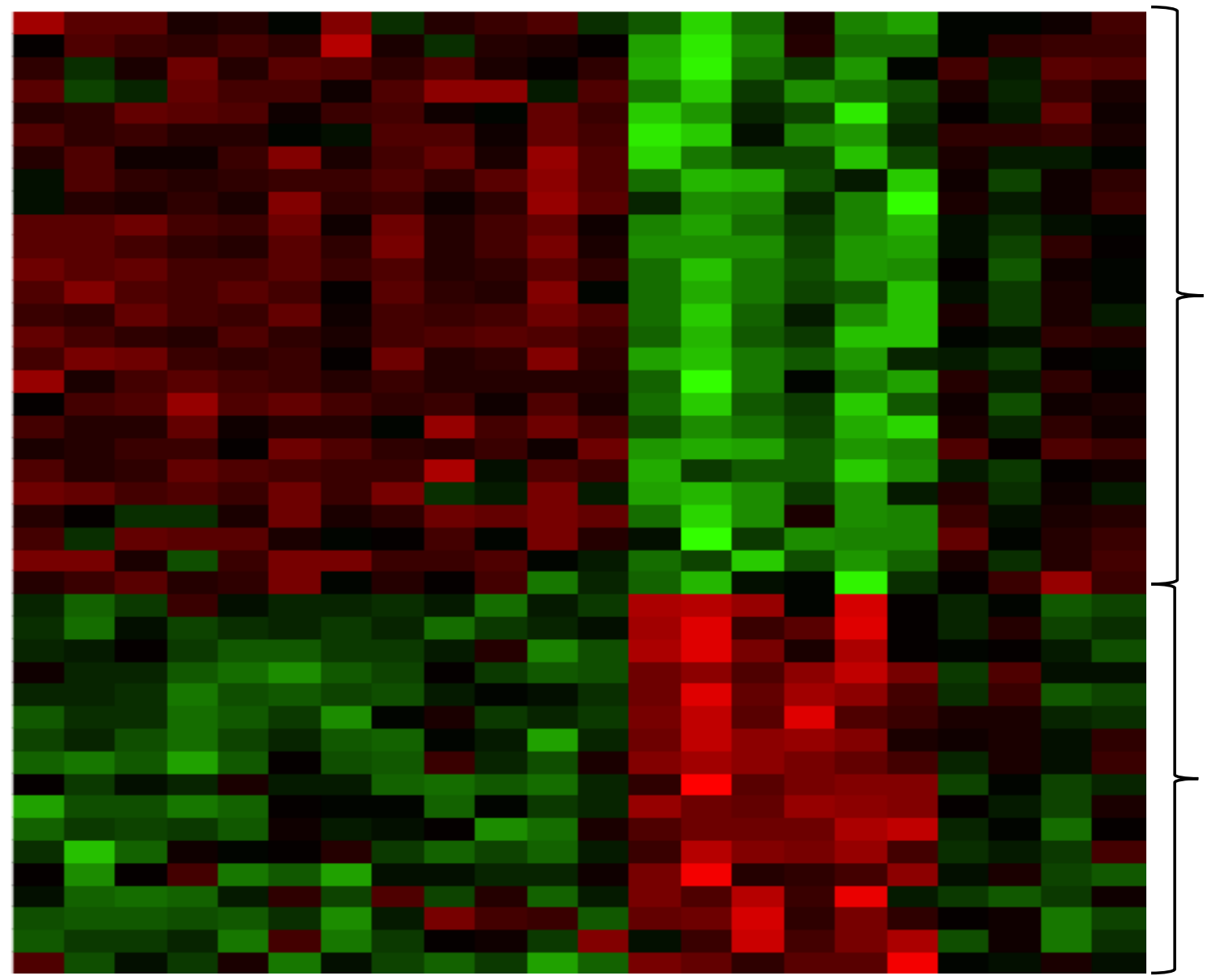

Young and Young/ERR

Old

Old/ERR

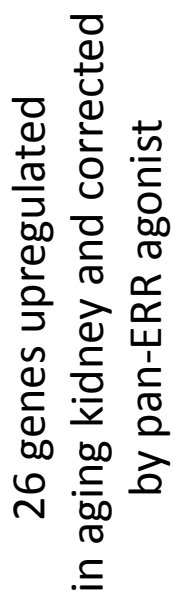

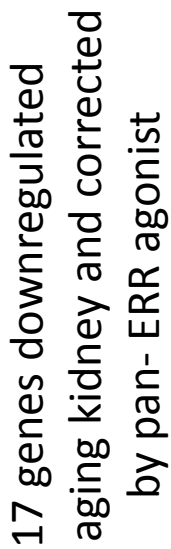

$\begin{array}{lll}-2 & 0 & 2\end{array}$

Row Z-Score

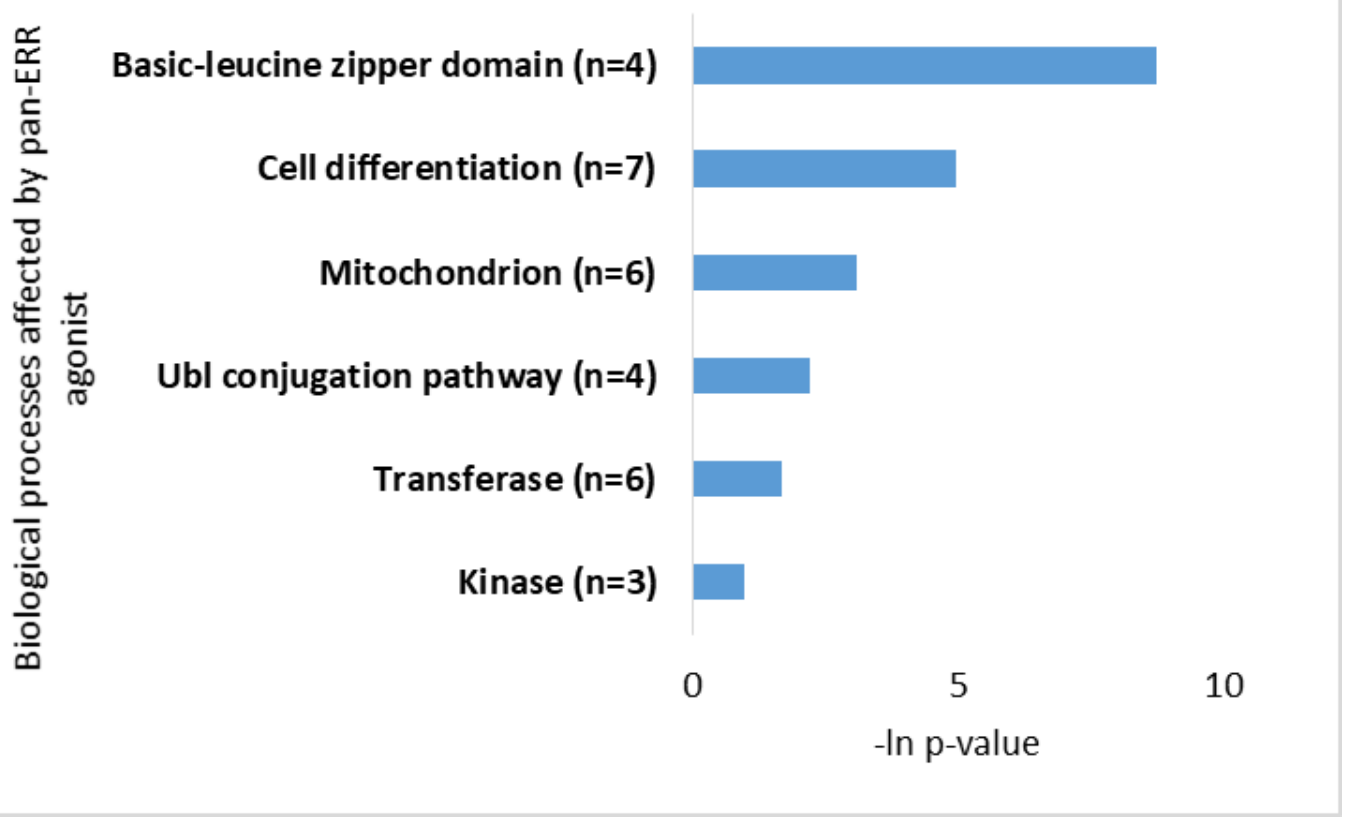




\section{Figure 4}
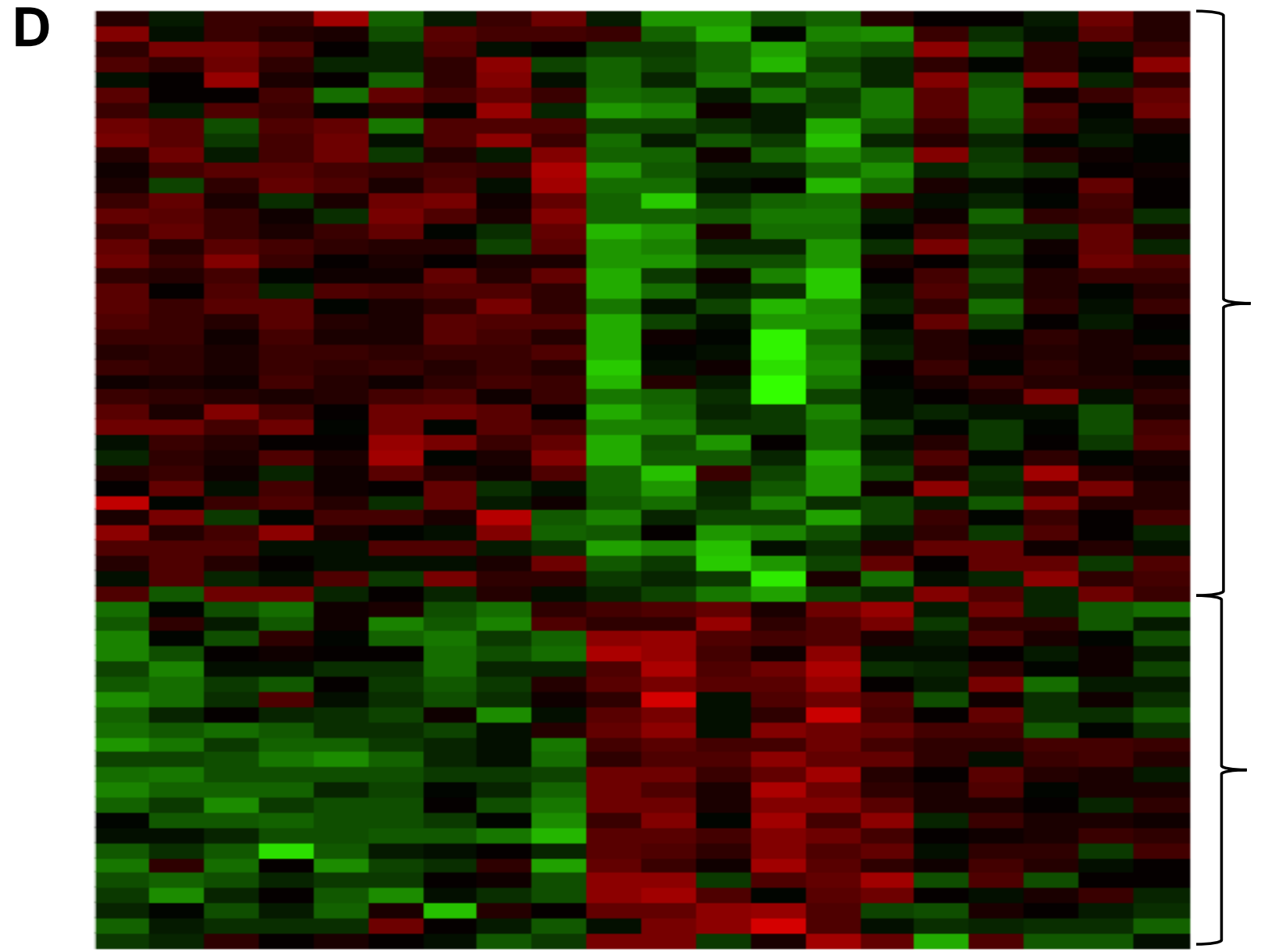

Young and Young/ERR

Old

Old/ERR

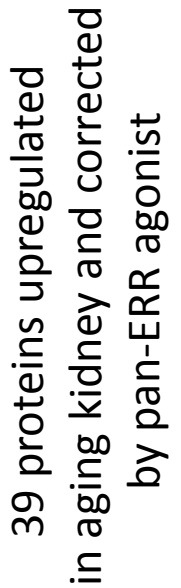

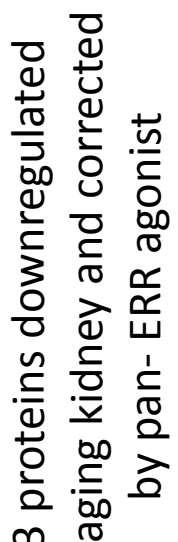

$\stackrel{\sim}{\sim}$

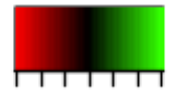

$\begin{array}{llll}-3 & -1 & 1 & 3\end{array}$

Row Z-Score

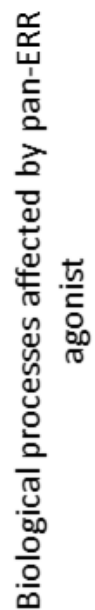

Mitochondrion $(n=13)$

Negative regulation of mRNA splicing $(n=4)$

Immunity ( $n=6)$

Antigen processing and presentation $(n=4)$

Transport $(n=11)$

Focal adhesion $(n=5)$

Fatty acid metabolism ( $n=3$ )

0 


\section{Figure 4}

$\mathbf{F}$

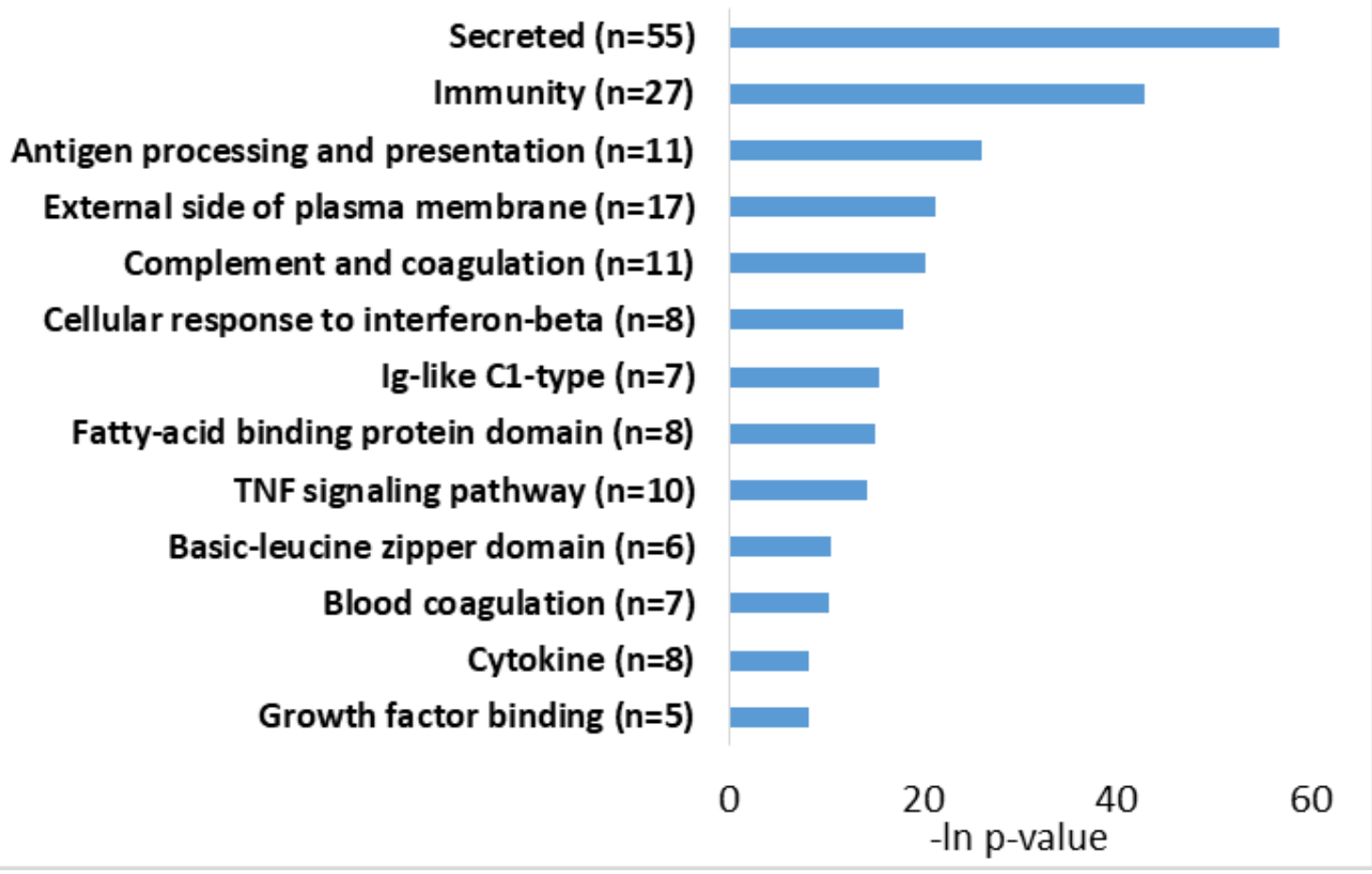

Lipid metabolism $(n=11)$

Fatty acid metabolic process $(n=6)$

Transmembrane transport $(n=8)$

Catalytic activity $(n=6)$

Calcium-binding region $(n=3)$

Endoplasmic reticulum $(n=7)$

$\begin{array}{cccccccccc}0 & 2 & 4 & 6 & 8 & 10 & 12 & 14 & 16 & 18 \\ \text {-In p-value } & & & & & & \end{array}$


Figure 5

A
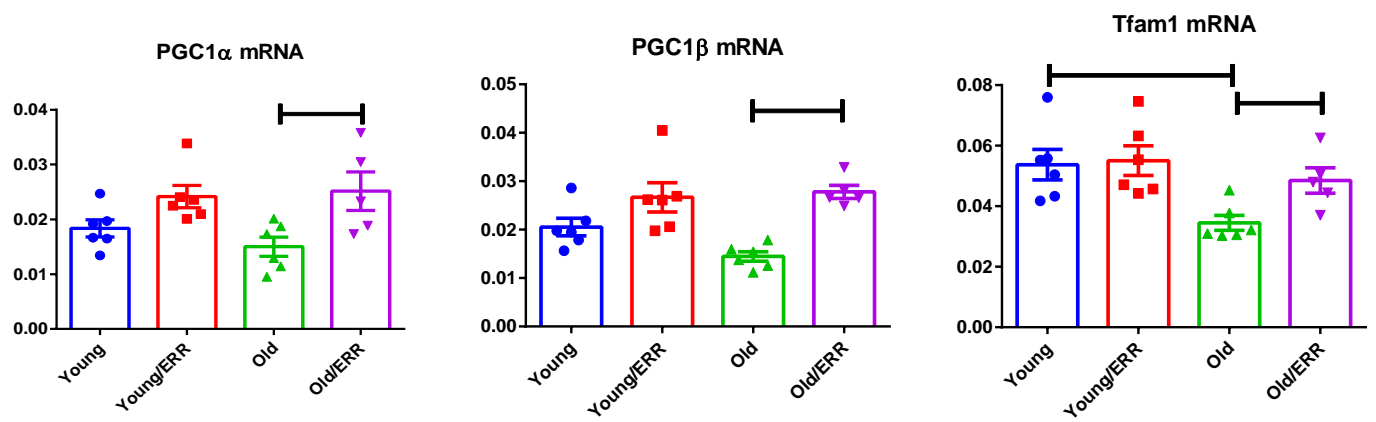

Mitochondria to nuclear

DNA ratio

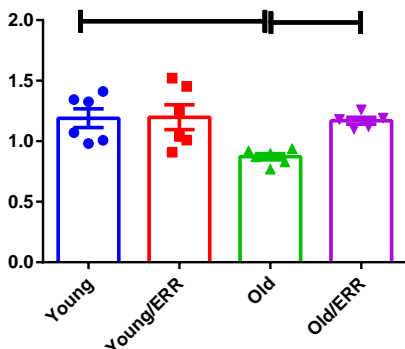

B

Complex I Ndufb8 mRNA

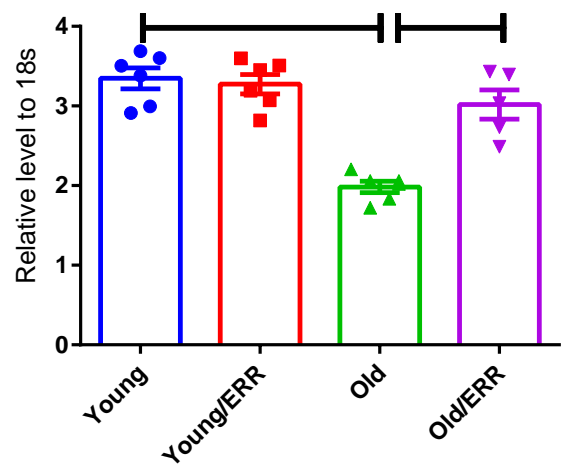

Complex IV Cox6a2 mRNA

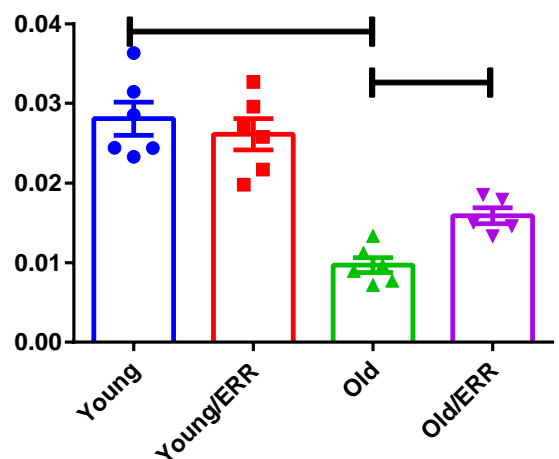

Complex II SDHC mRNA

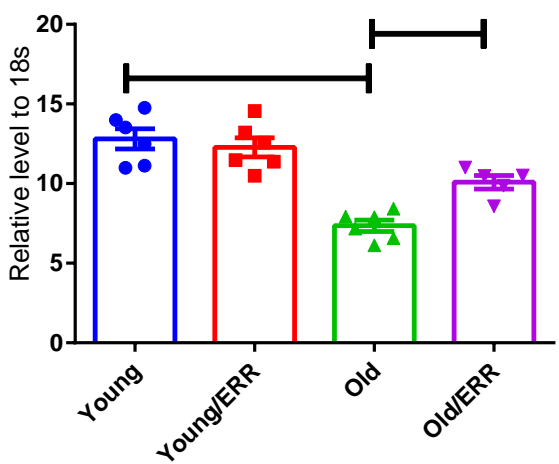

Complex V ATP5b mRNA

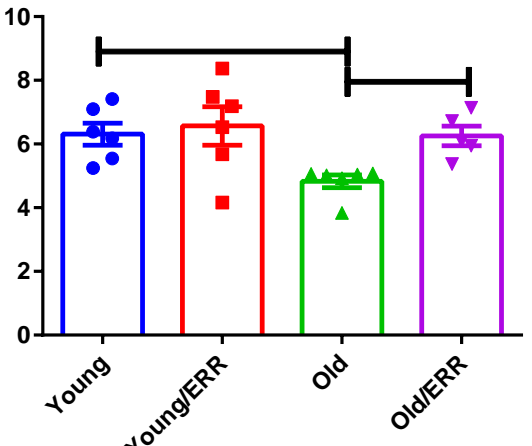

Complex III Uqcrb mRNA

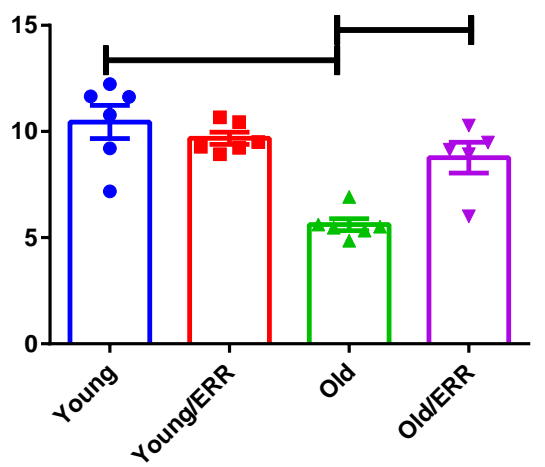


Figure 5

C

PDHB mRNA

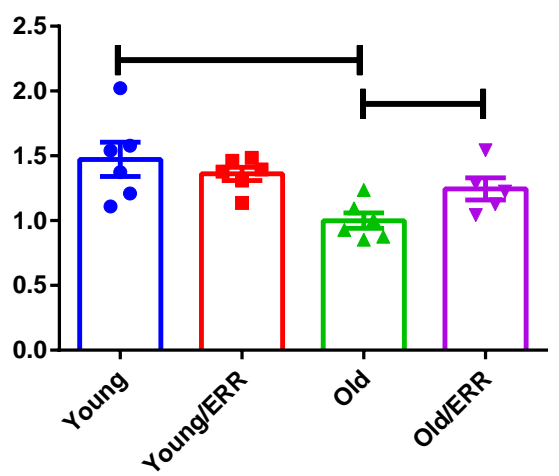

SUCLA2 mRNA

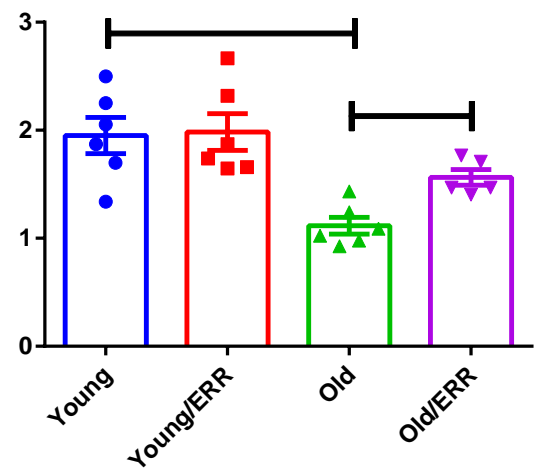

MDH1 mRNA

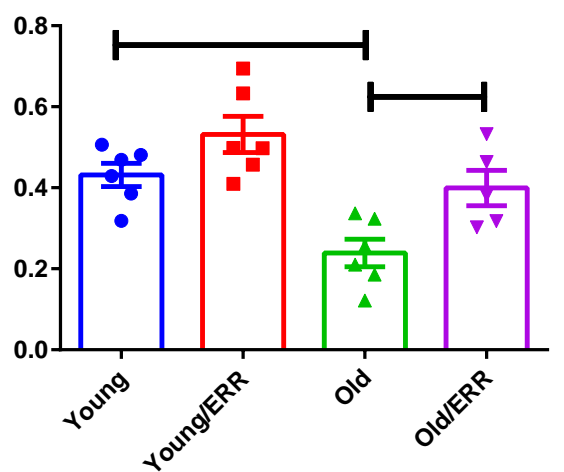

IDH3b mRNA

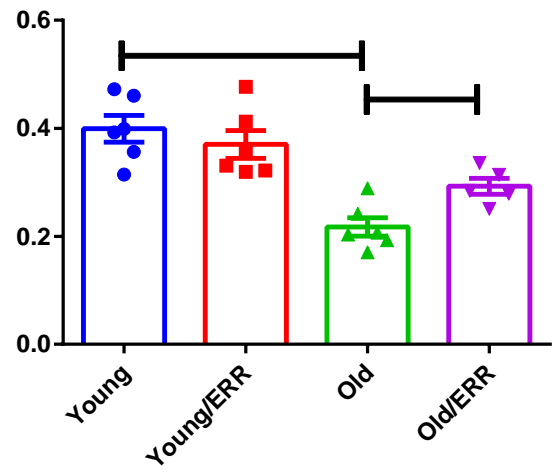

D
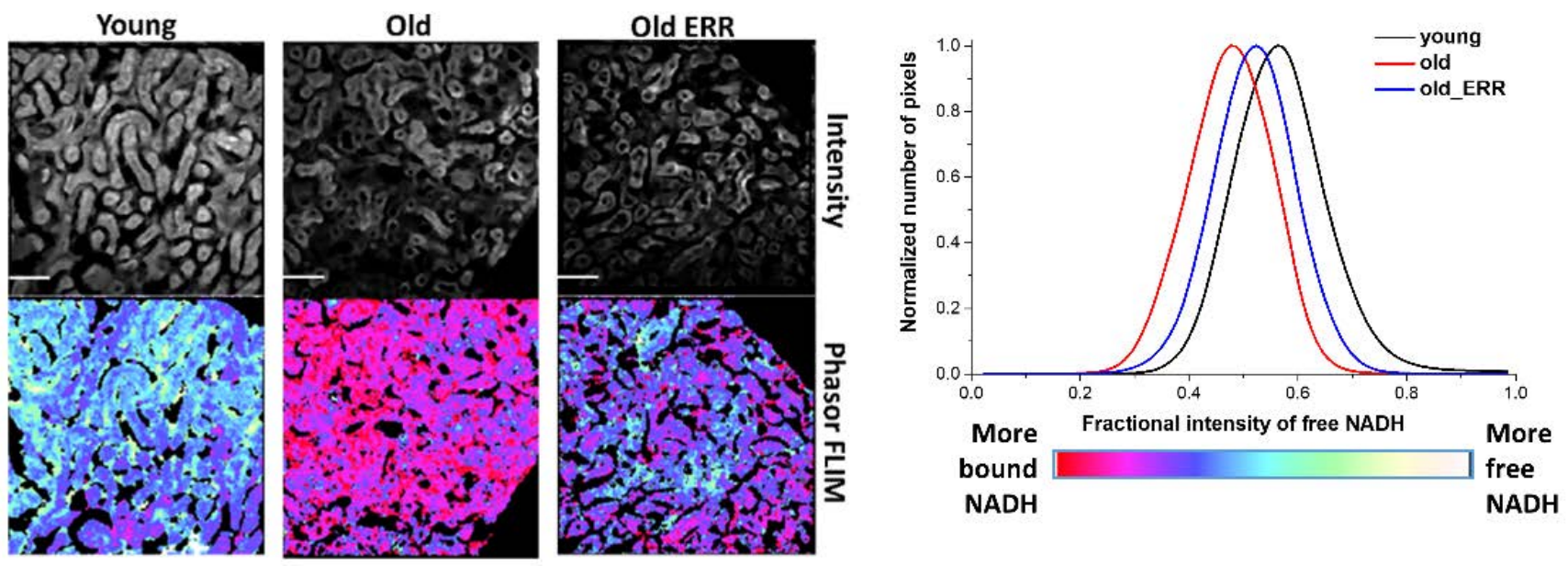
Figure 5

E

\section{Glycolysis}

Electron Pan-ERR

Transport $\stackrel{\perp}{\Perp}$ Aging Chain
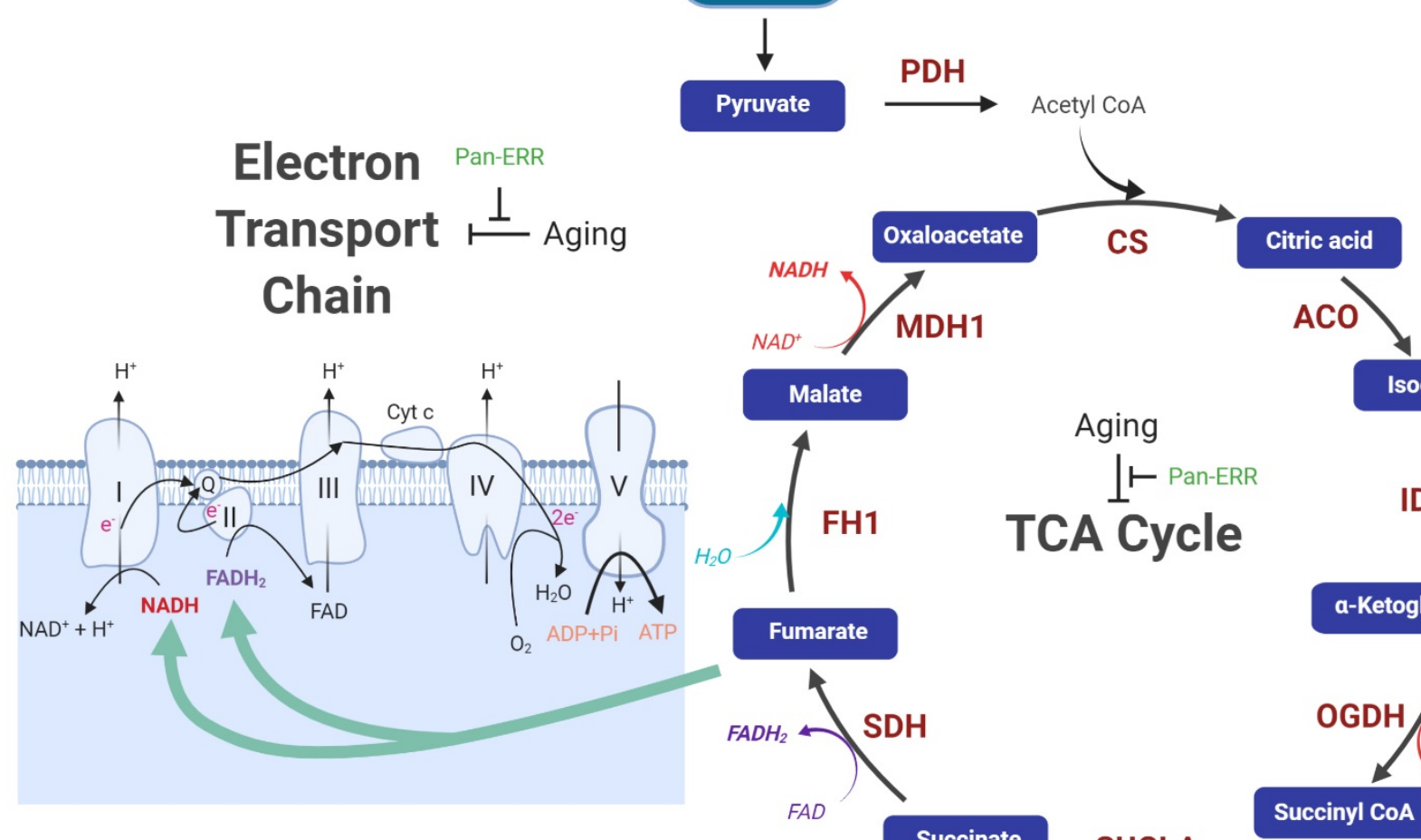

Isocitric acid

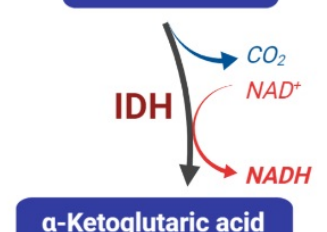

a-Ketoglutaric acid

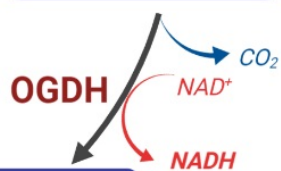

Succinate

Succinyl CoA

$\mathbf{F}$

Kidney Maximum Respiration Capacity

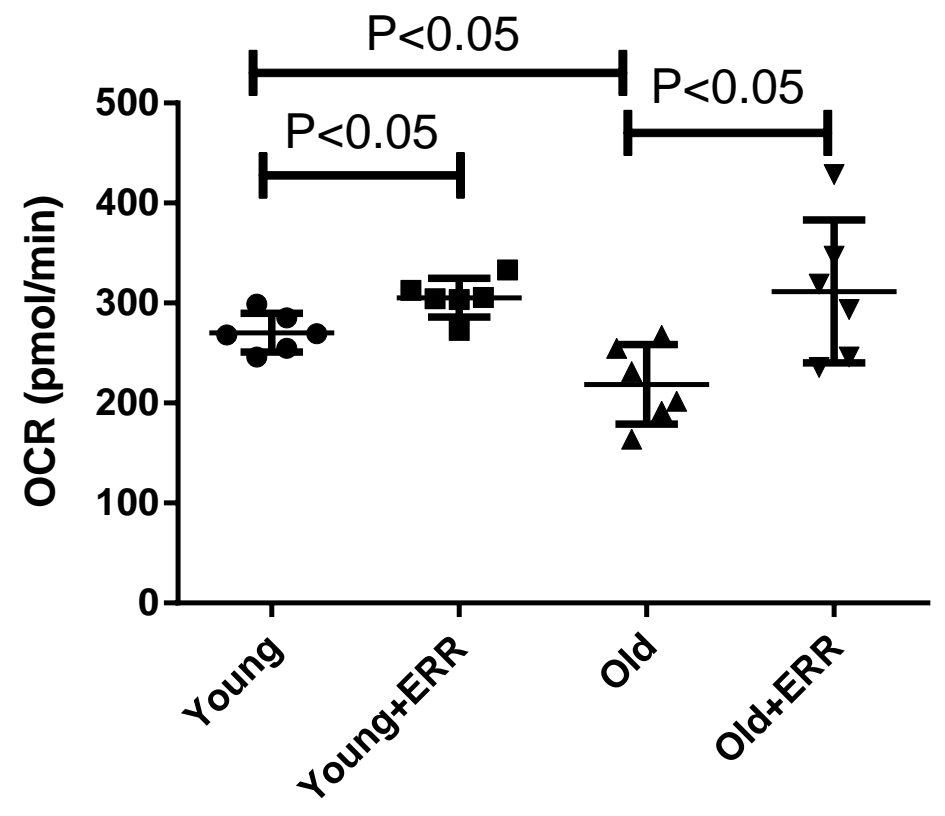


Figure 5

G

Cpt1a mRNA

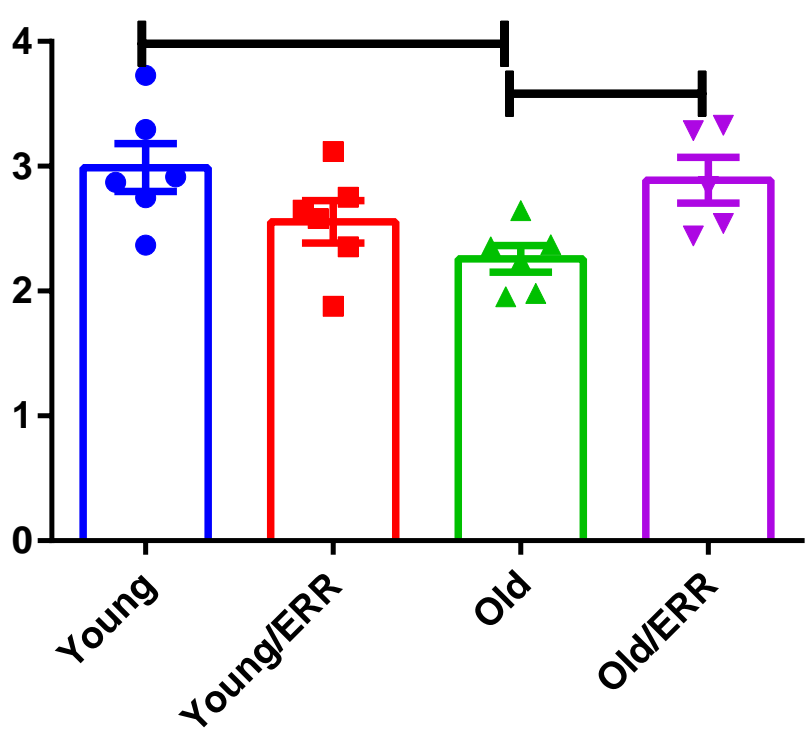

MCAD mRNA

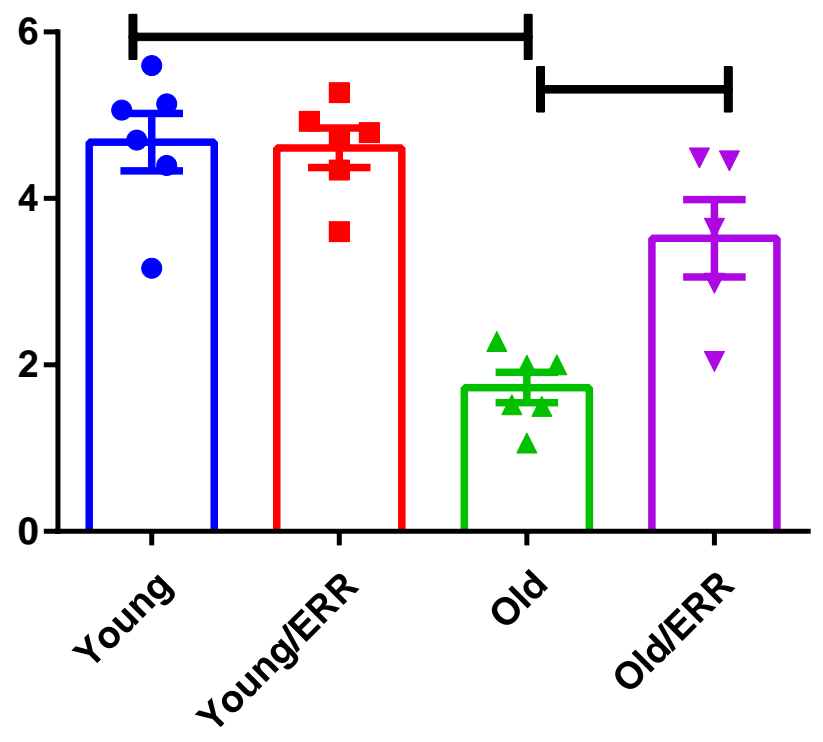


Figure 6
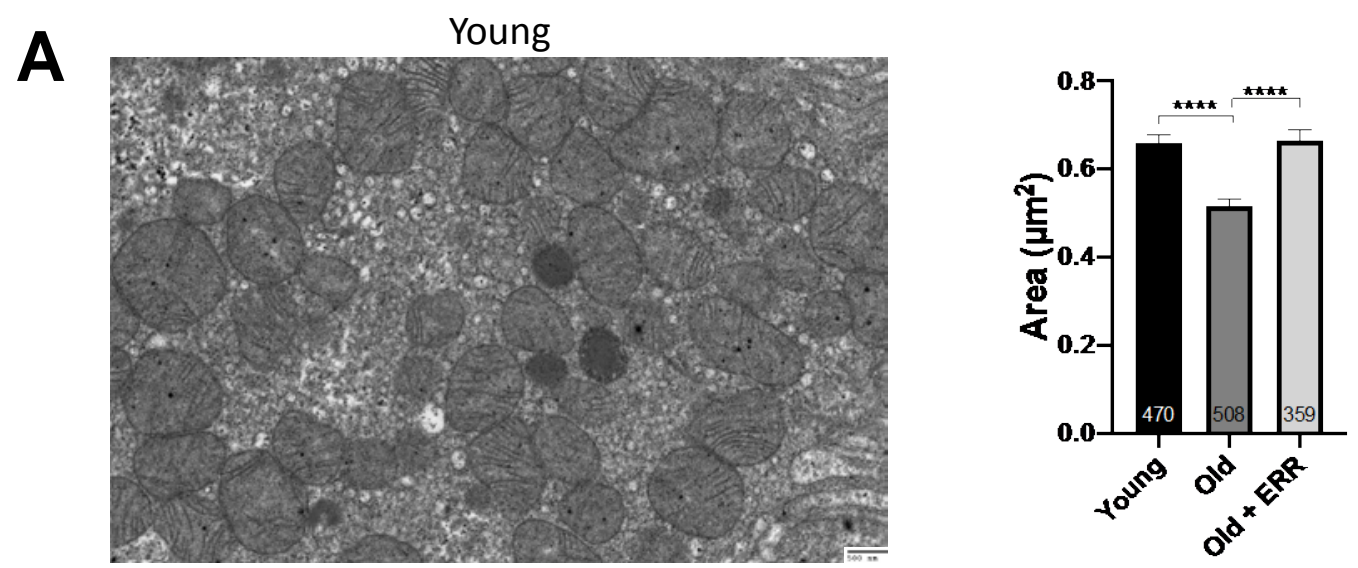

Old
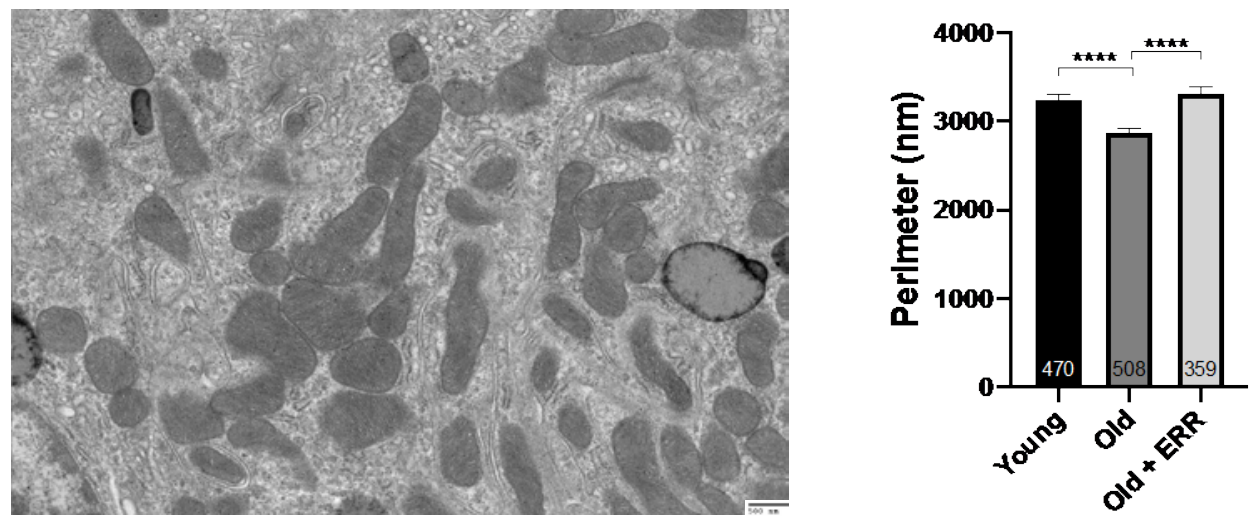

Old + ERR
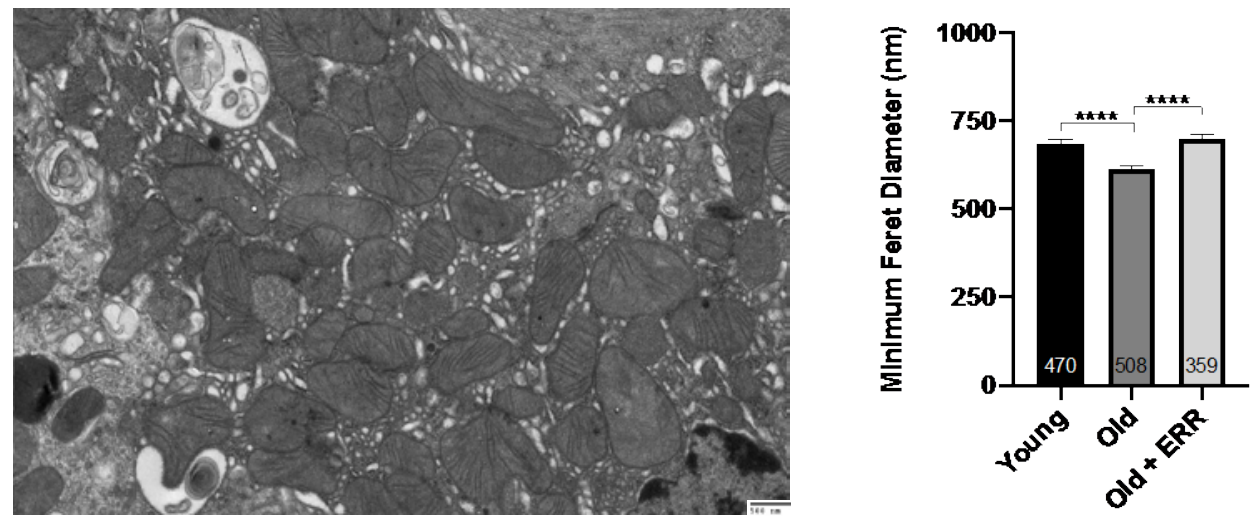
Figure 6

B
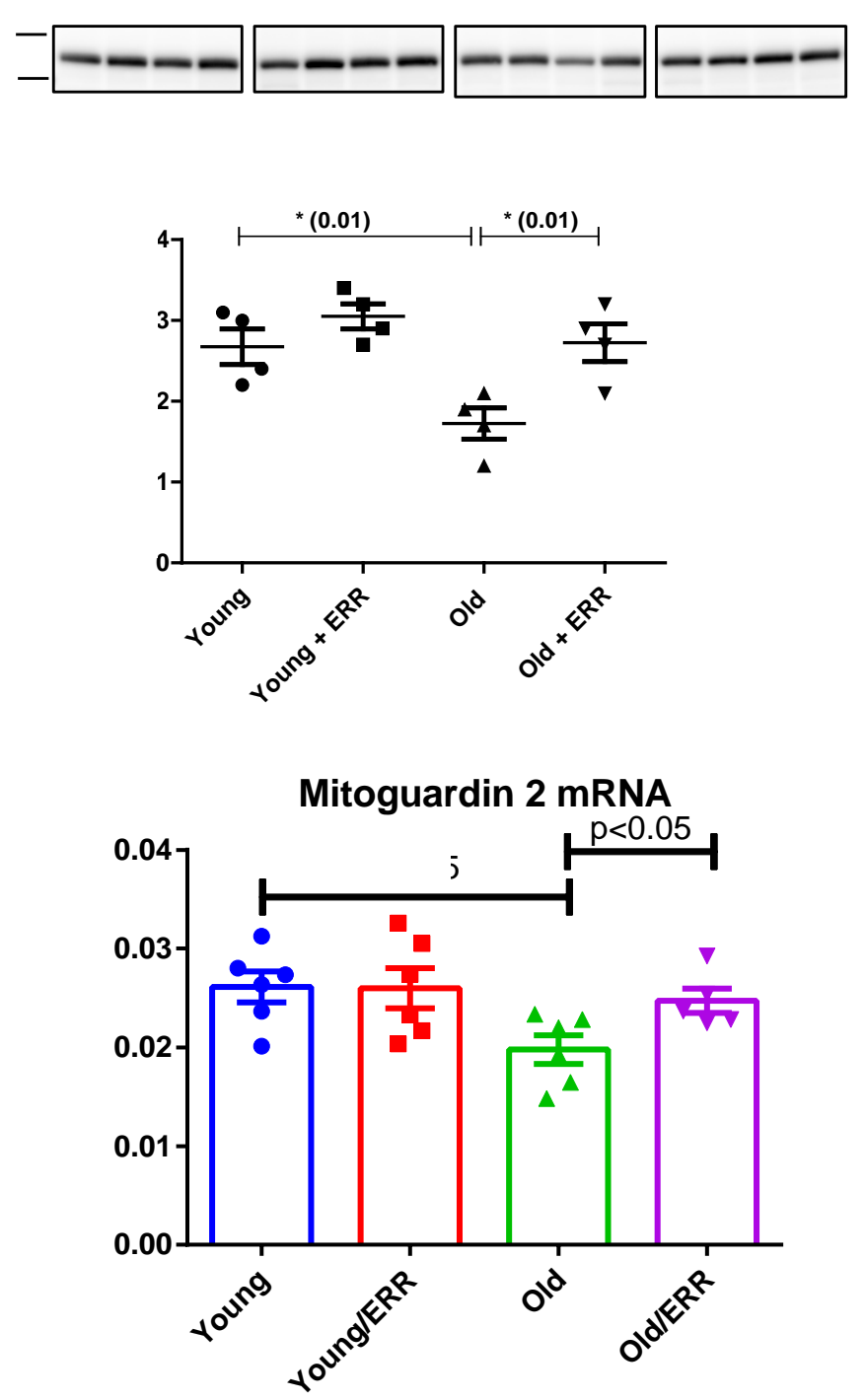

Young Young + ERR Old Old + ERR
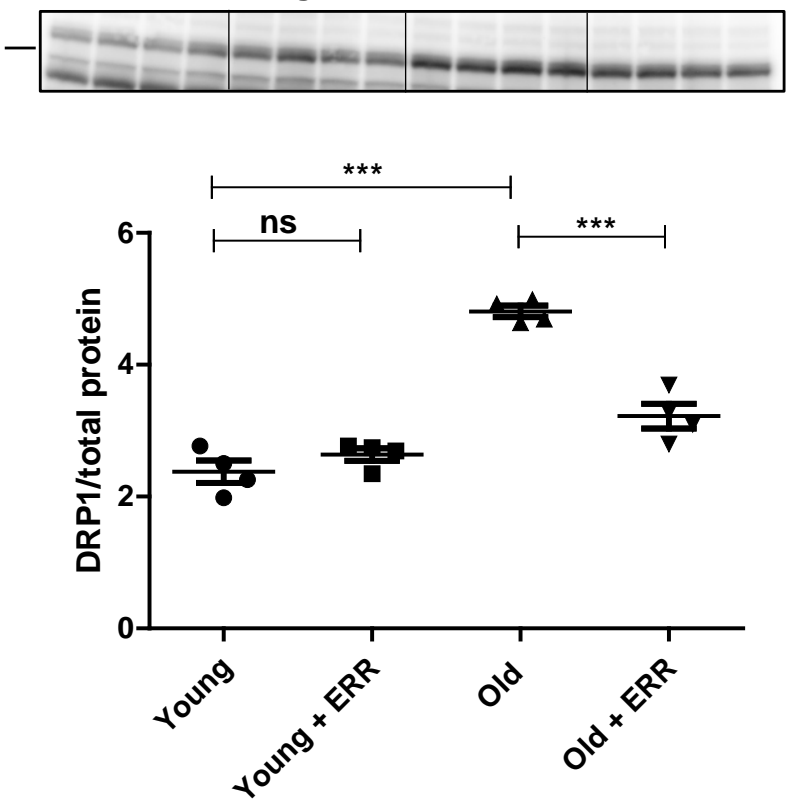
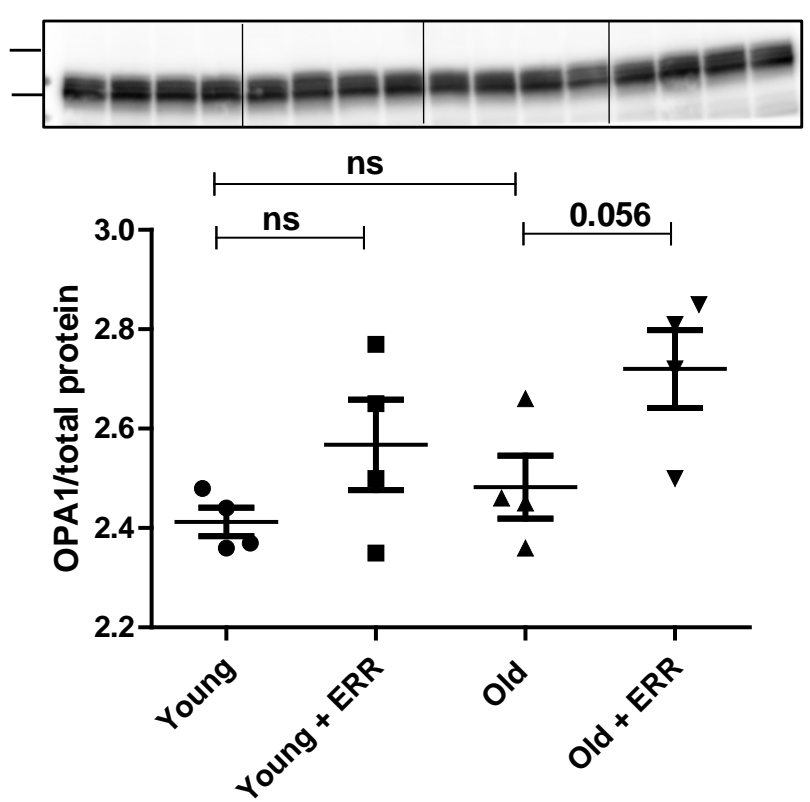

MitoPLD mRNA
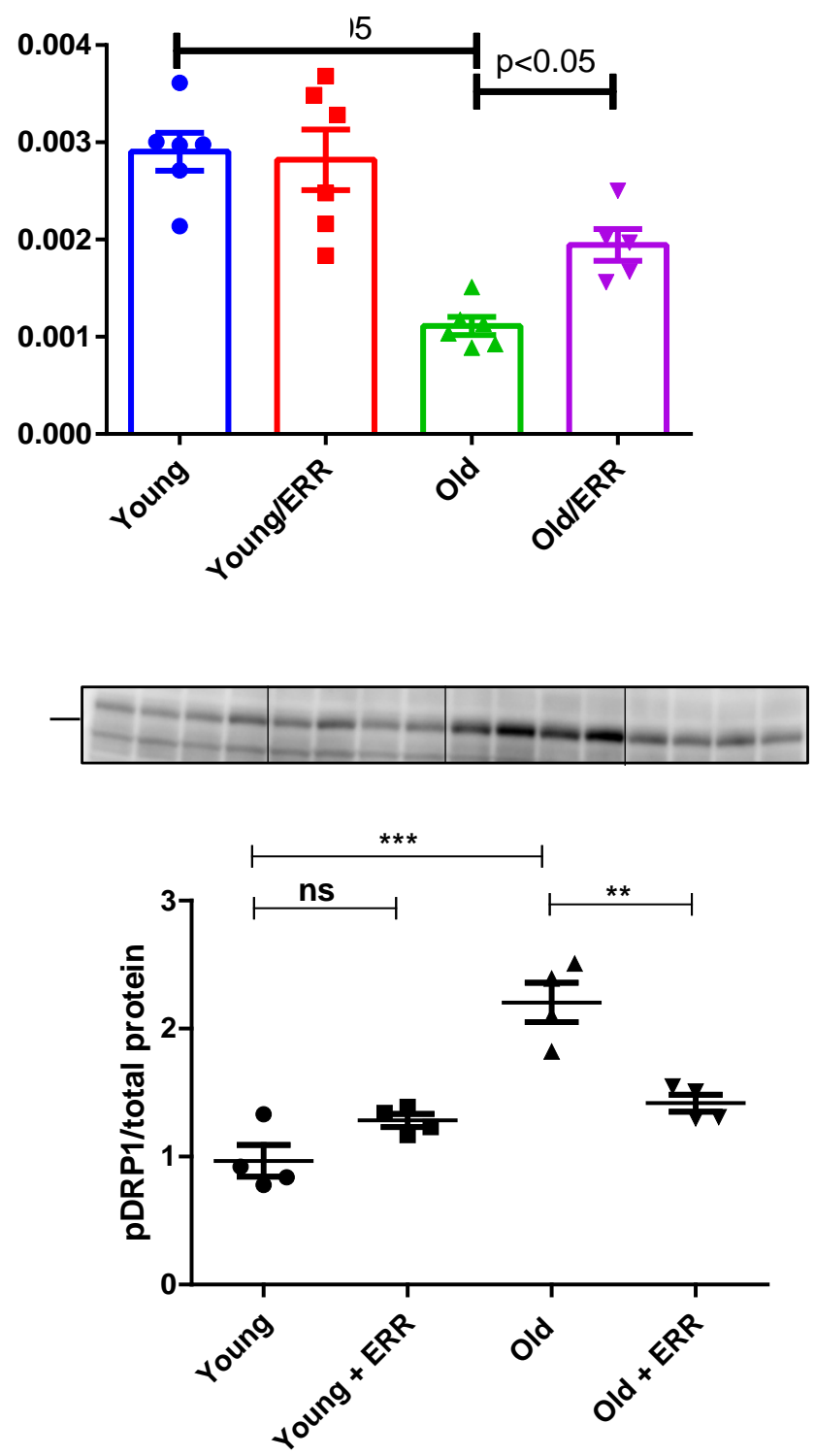
Figure 7

cGAS mRNA

$\mathbf{A}$

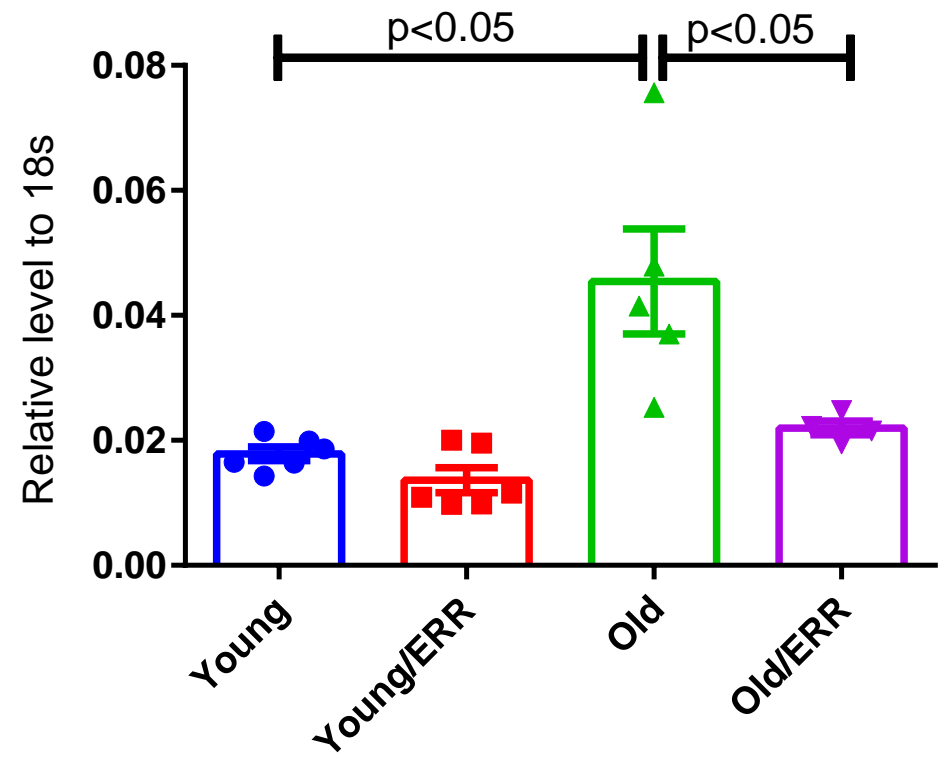

cGAS protein

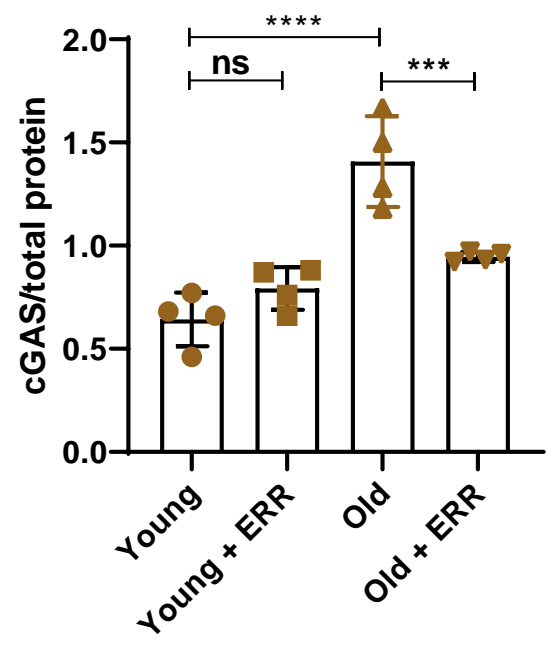

STING mRNA
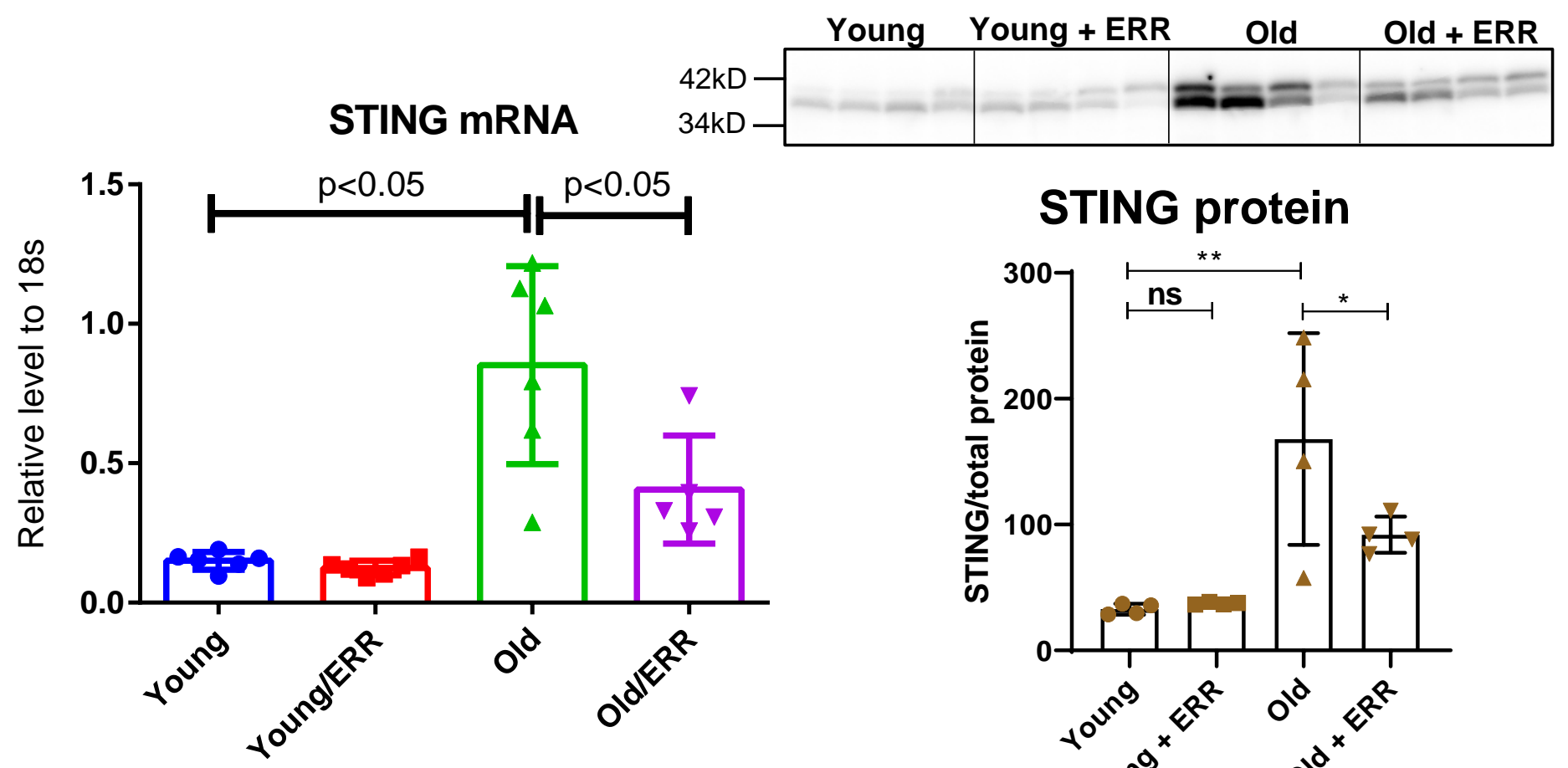

\section{STING protein}

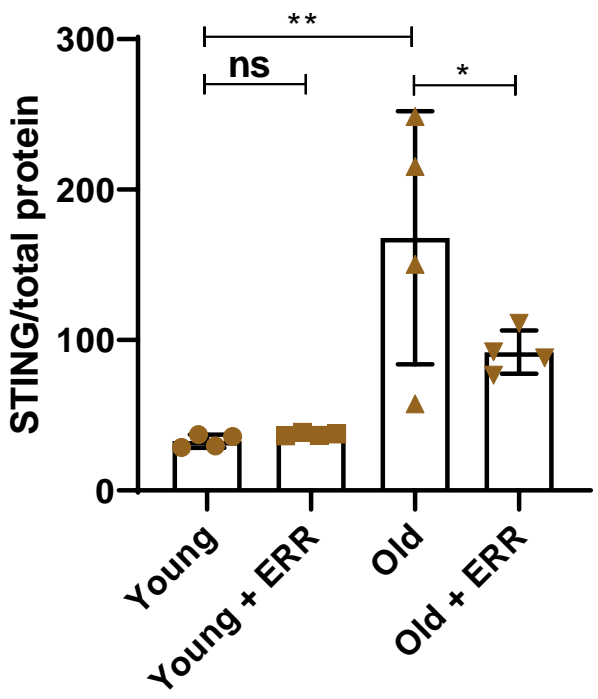


Figure 7

RIG-I mRNA

MDA5 mRNA
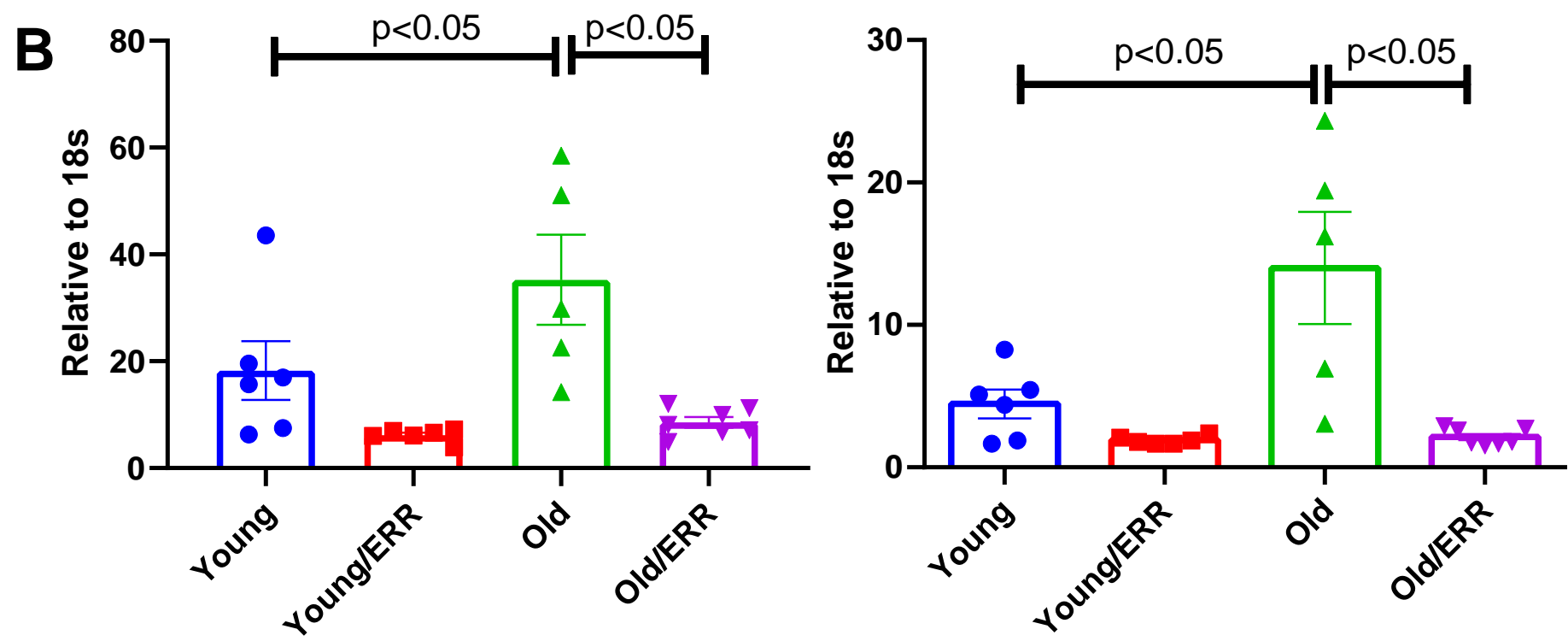

LGP2 mRNA

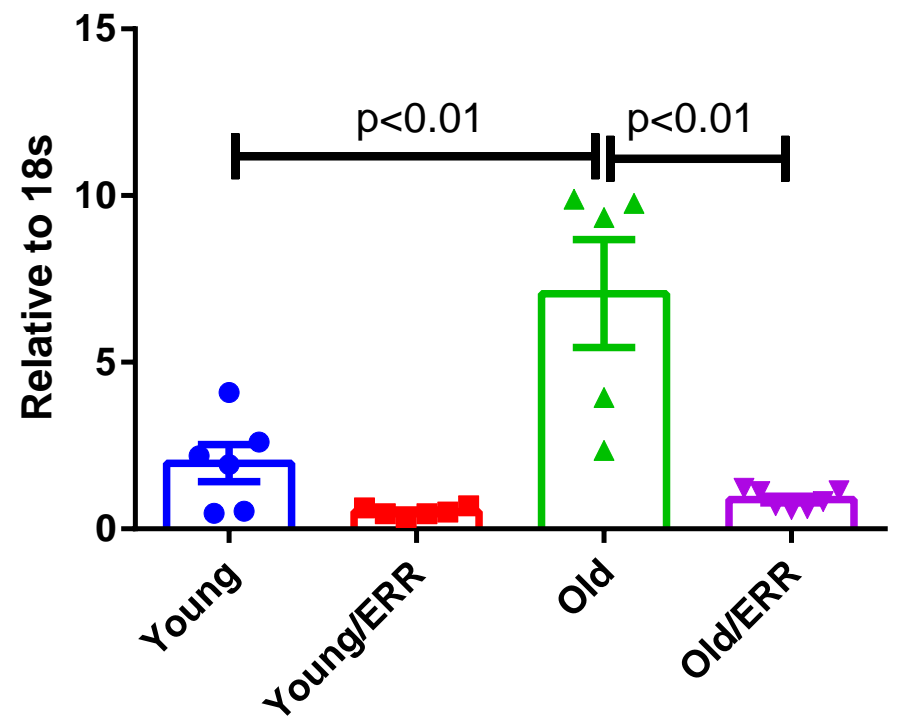

TLR7 mRNA
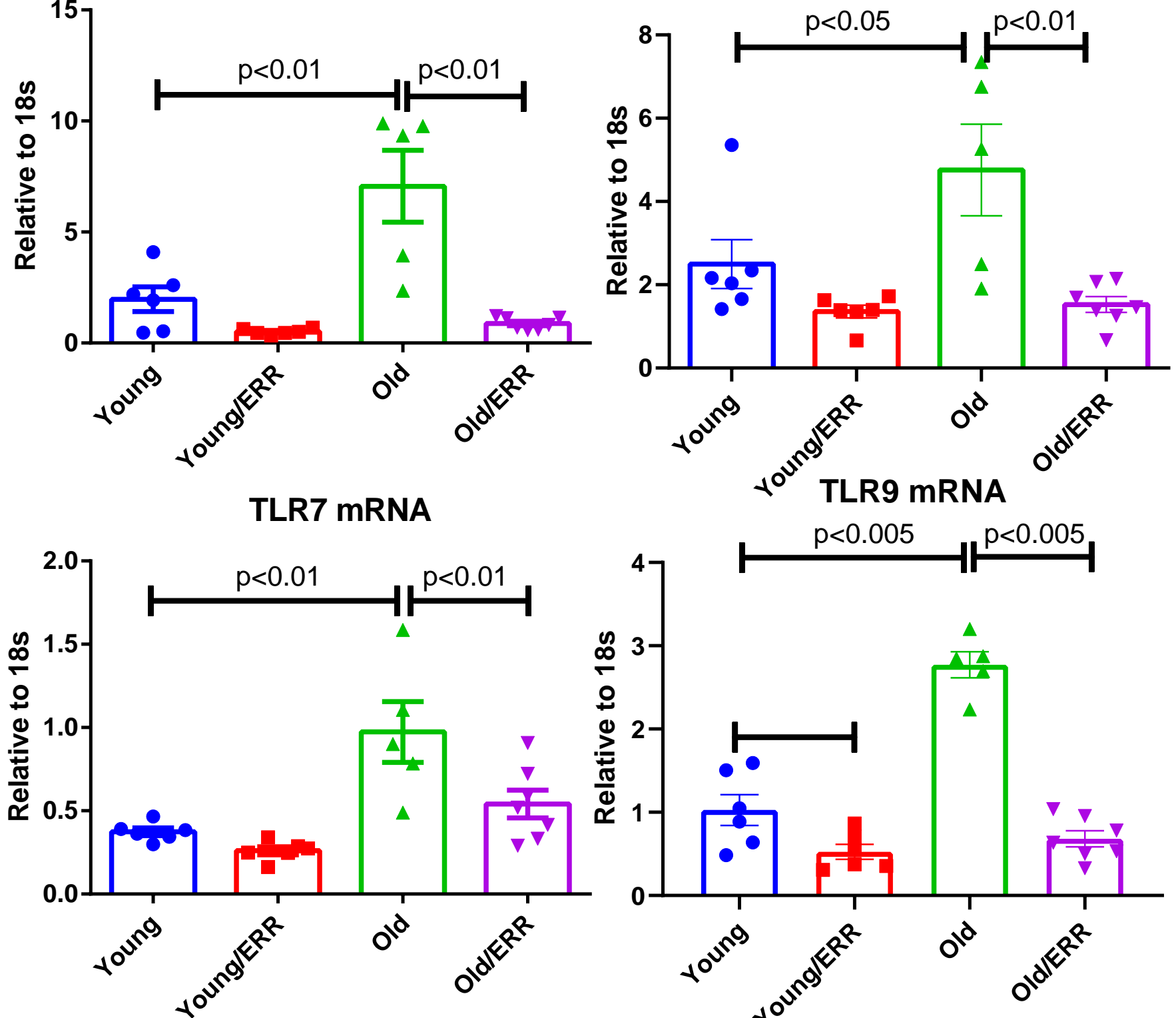
C
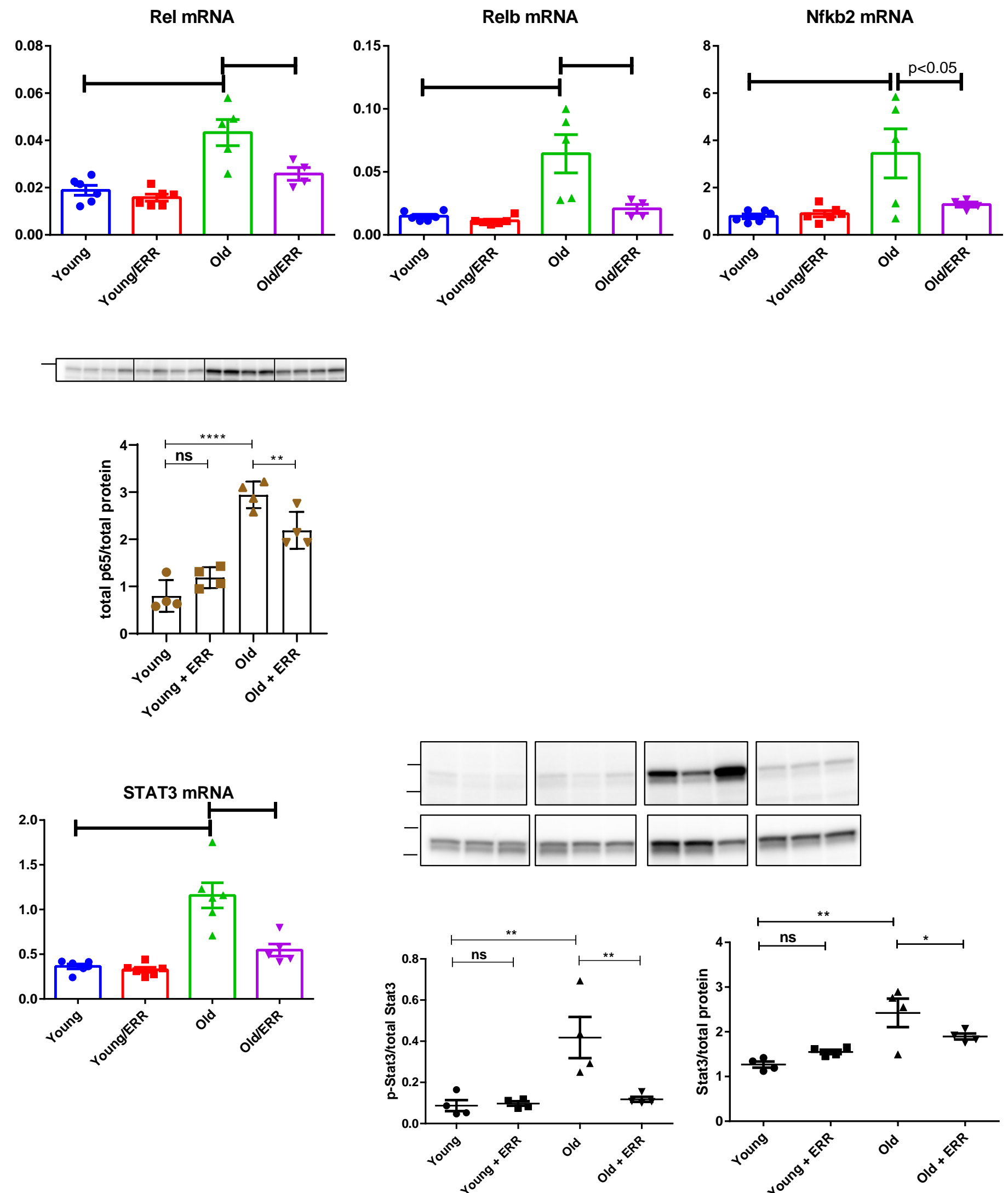
Figure 7

D

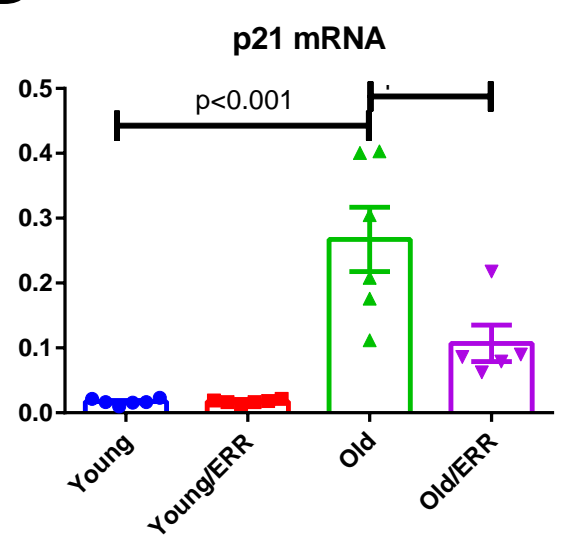

ICAM1 mRNA

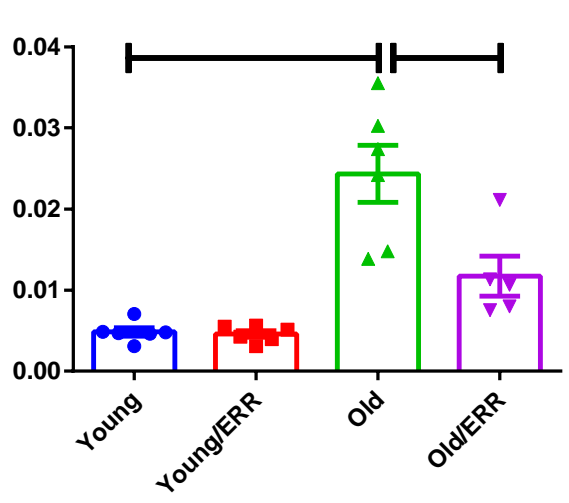

TNFa mRNA

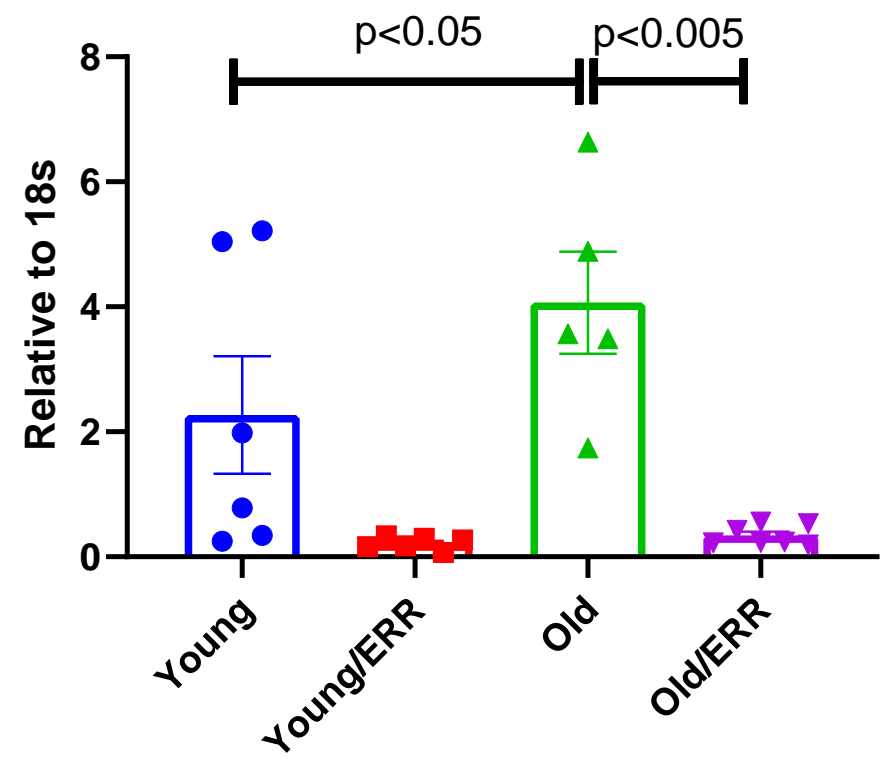

p16 mRNA

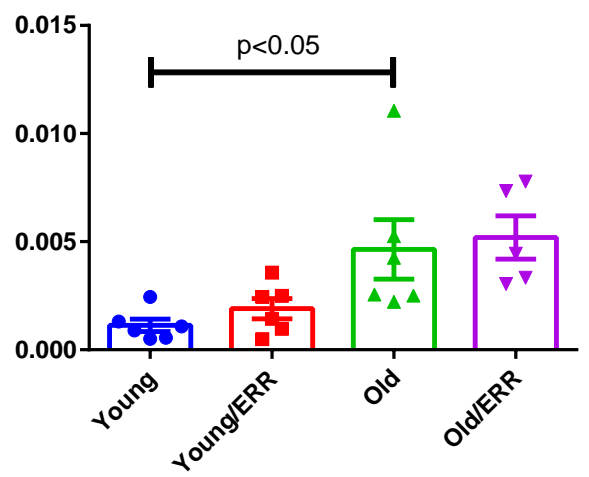

Timp1 mRNA

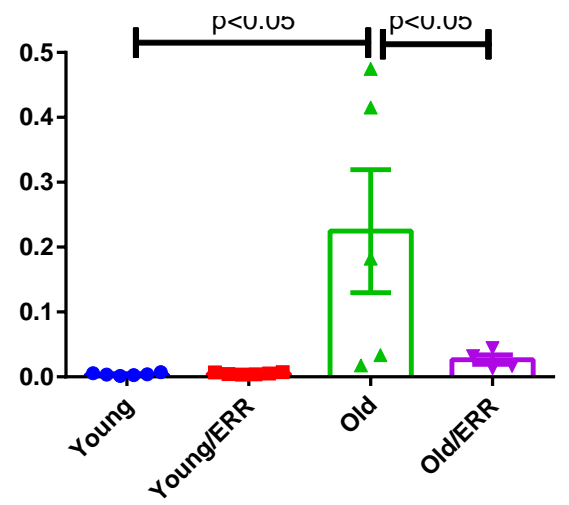

IL-1 $\beta$ mRNA

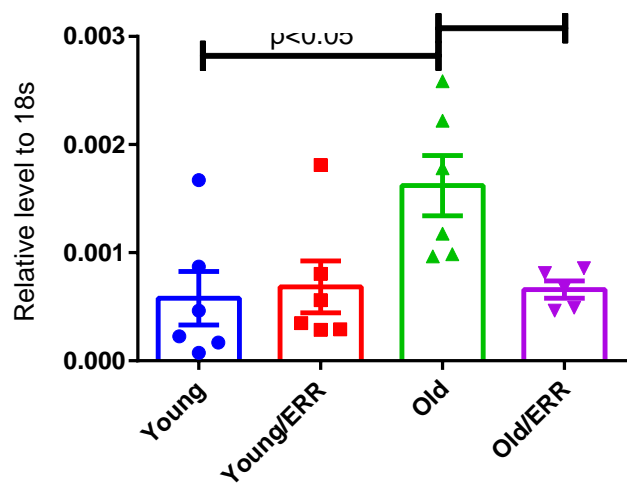

CCR5 mRNA

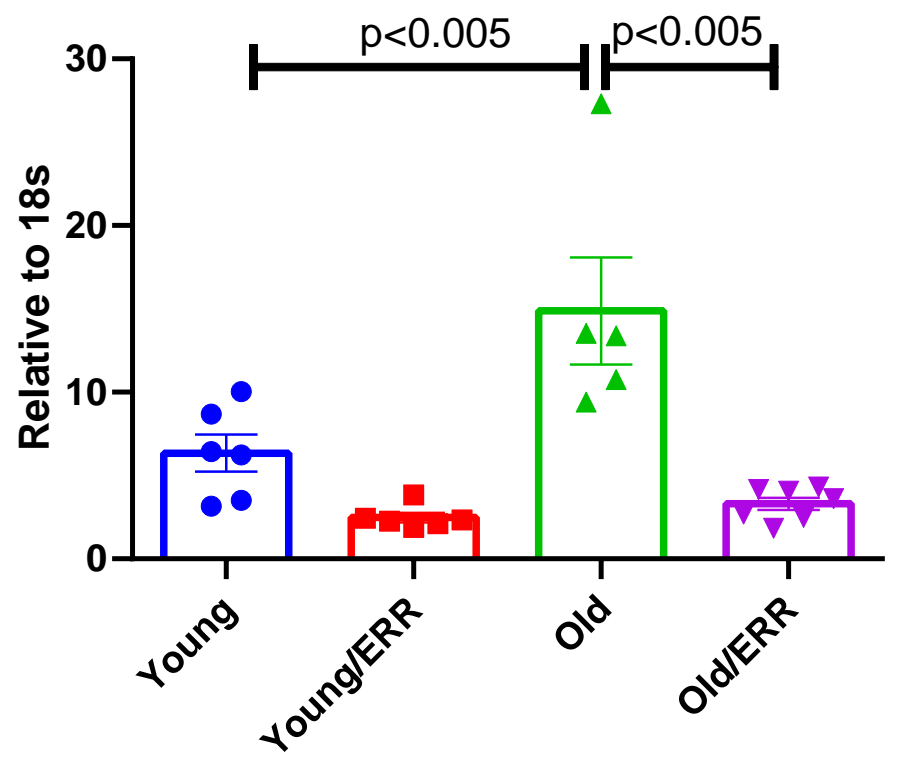


Figure 8

A

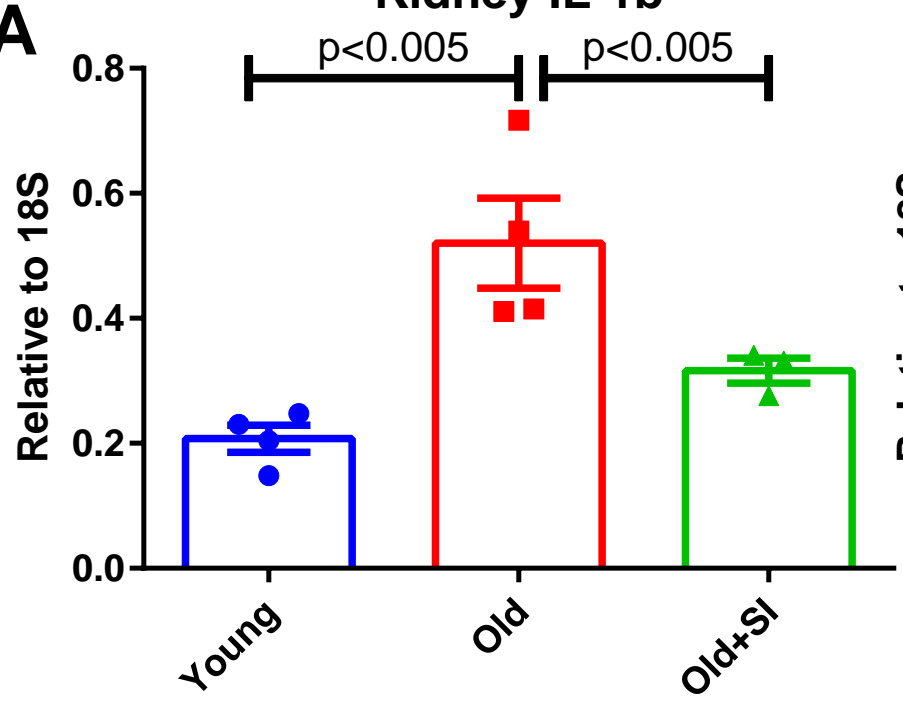

Kidney Stat3

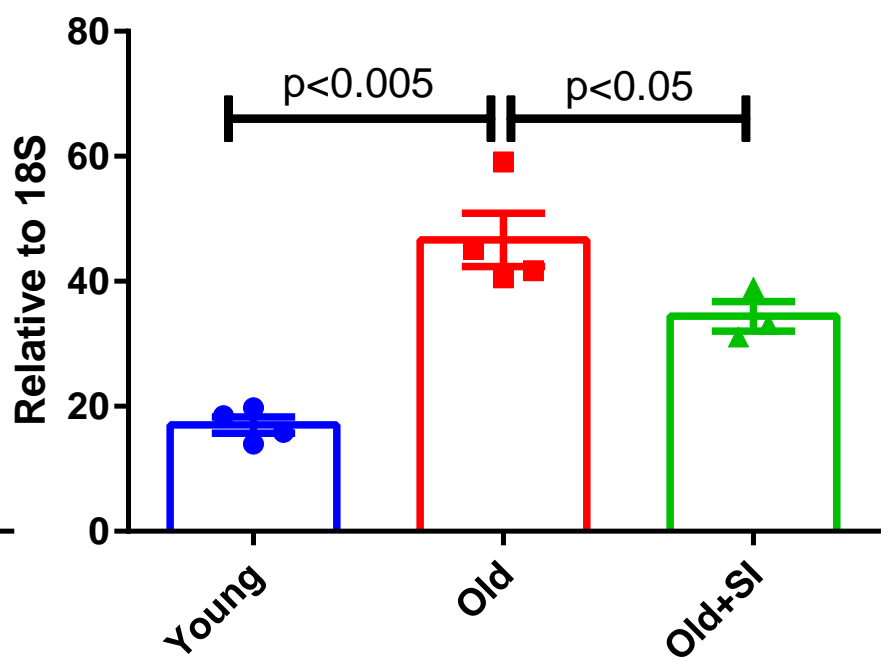

Young
Old
Old+Sting Inhibitor

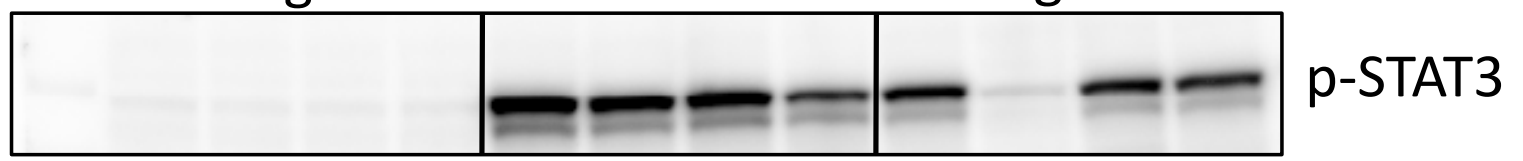

p-Stat3
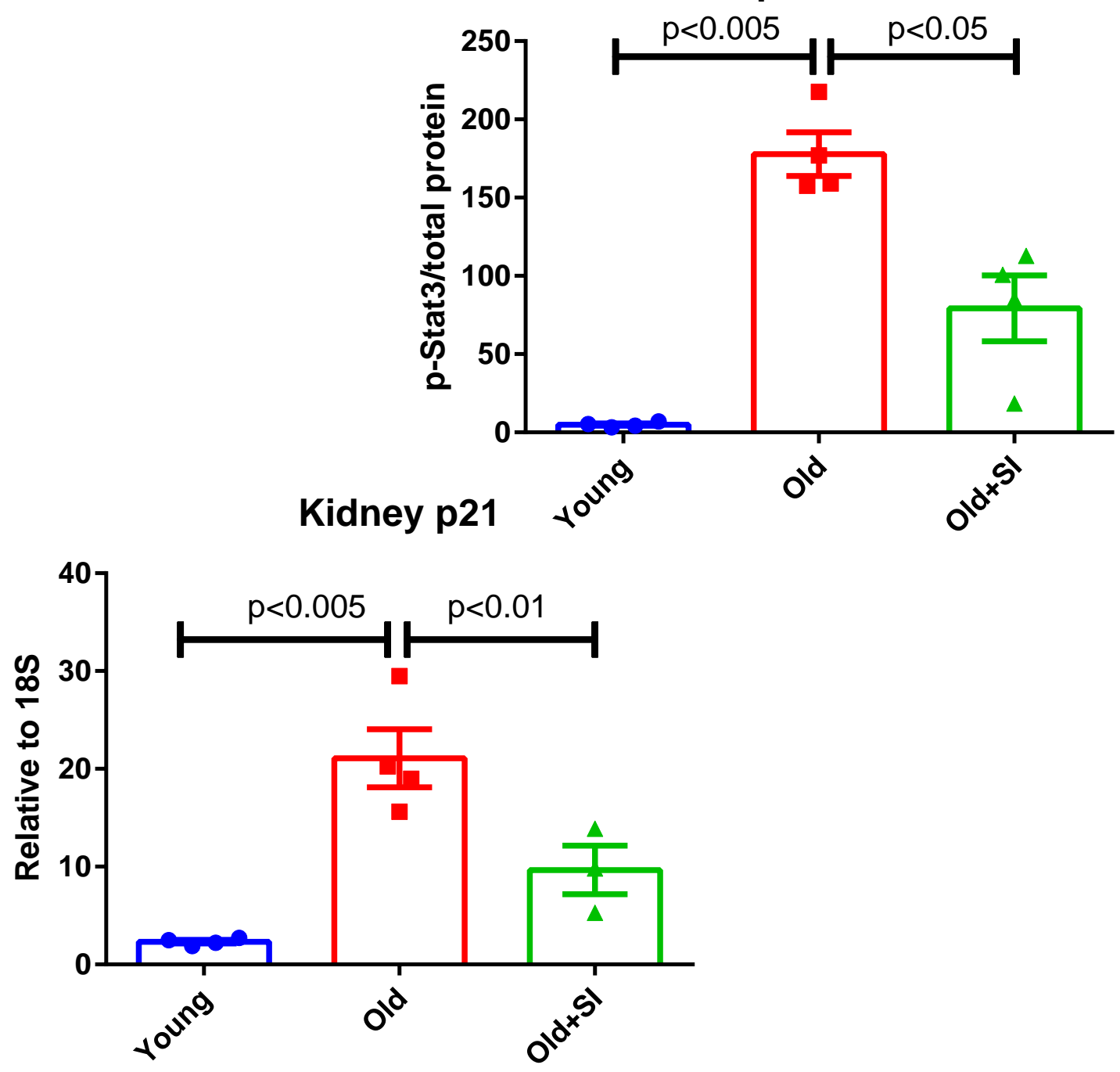
Figure 8

B

Kidney PGC1a

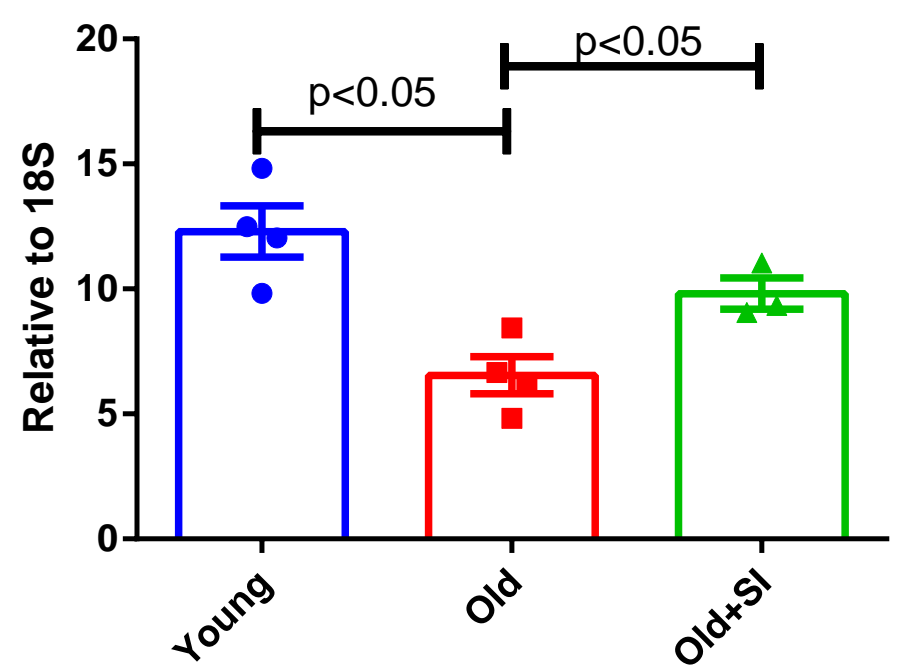

Kidney PGC1b

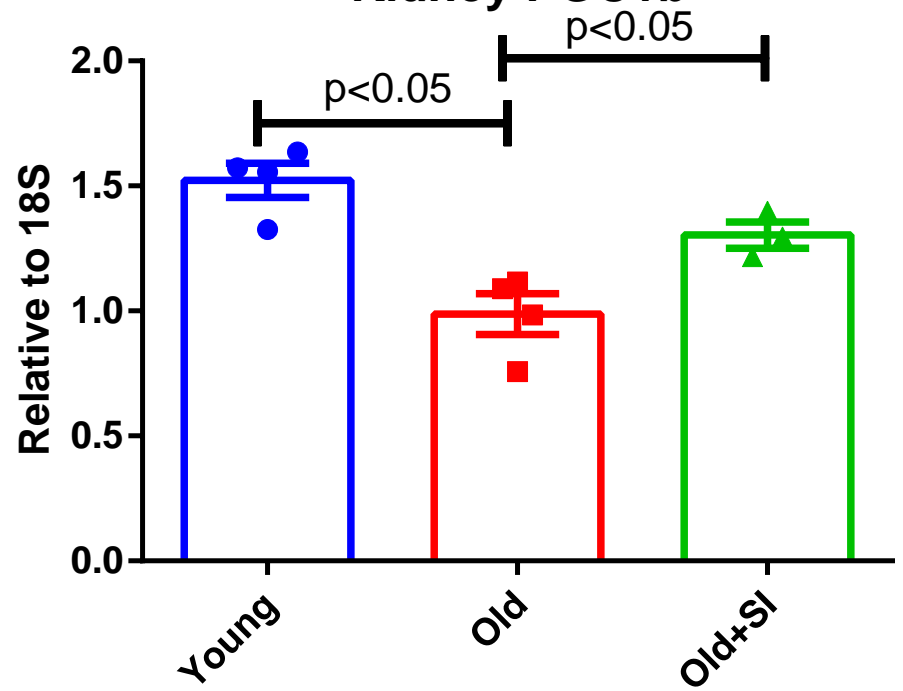

Old Old+Sting Inhibitor
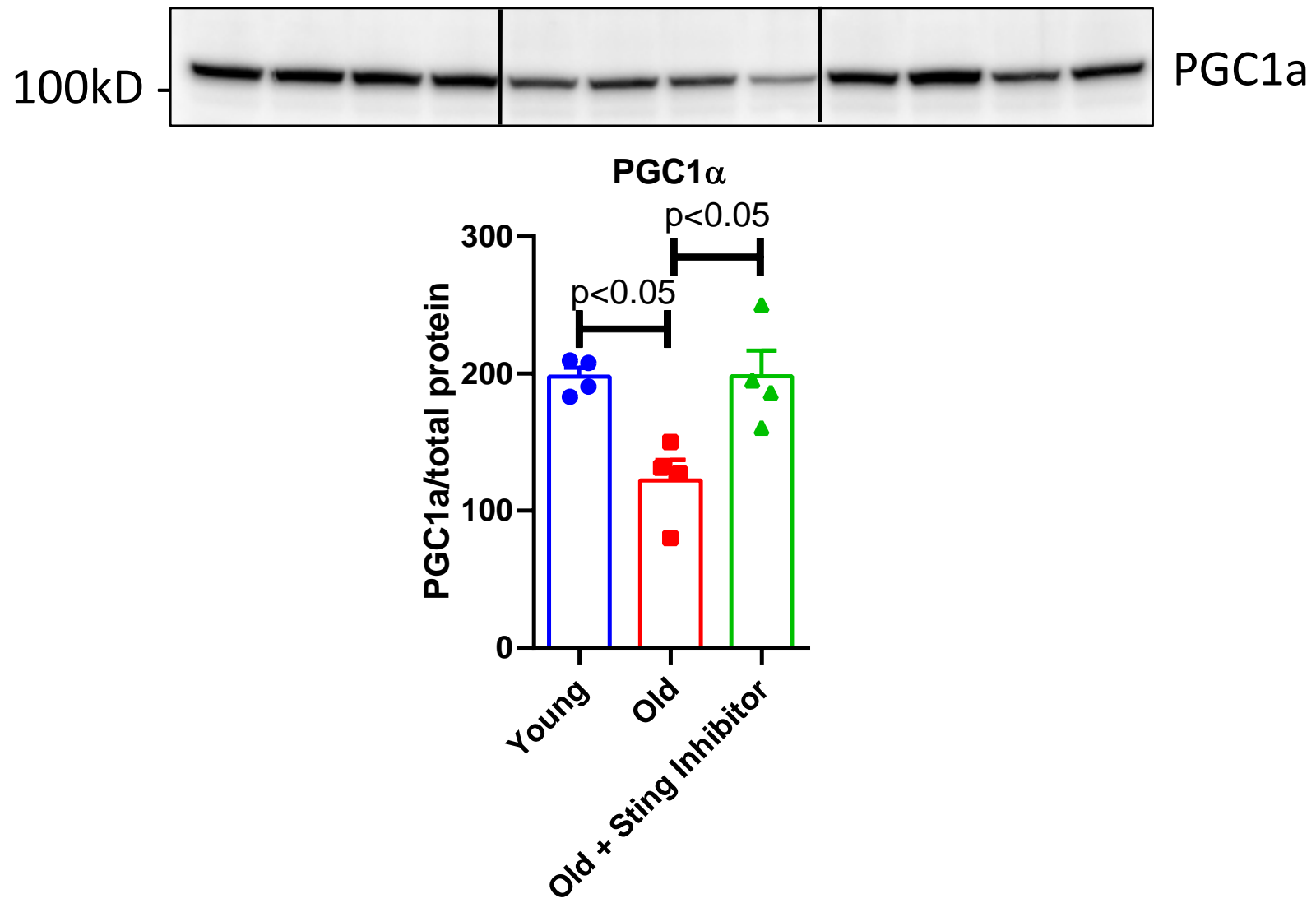
Figure 8

B

Kidney ERRa

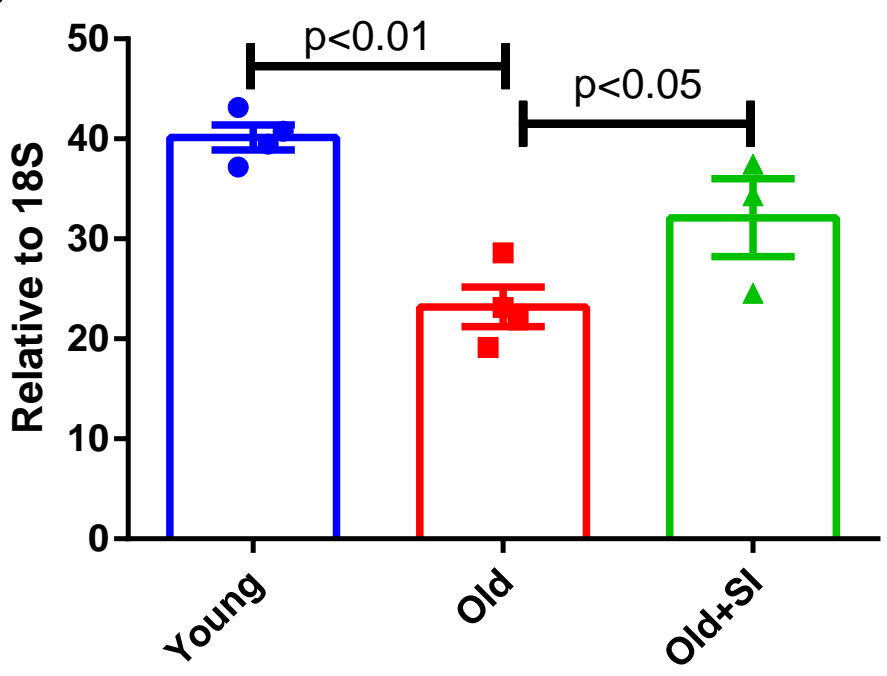

Cox6a2 mRNA

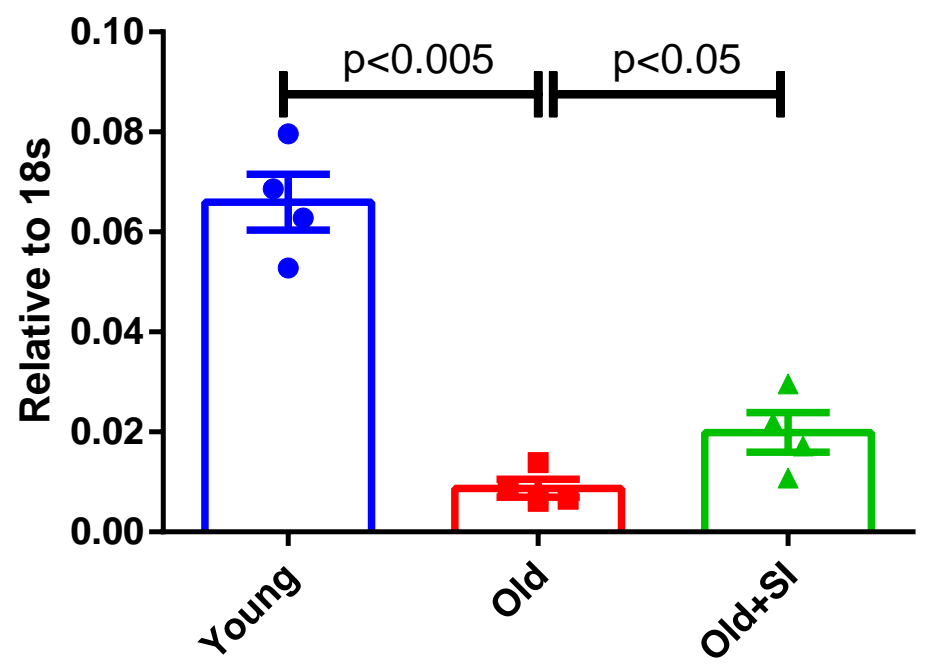

MCAD mRNA

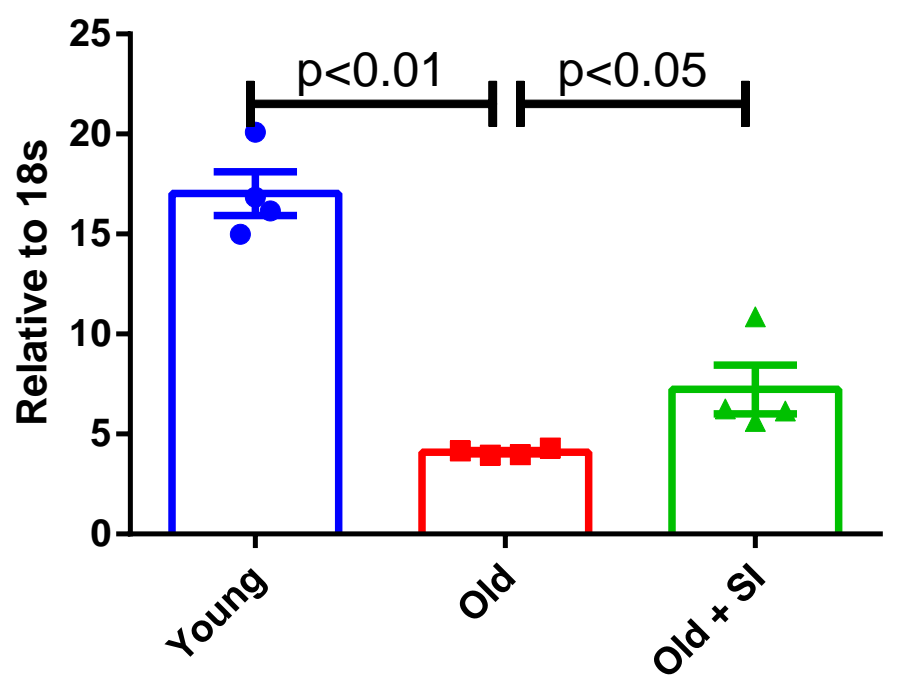

Ndufs1 mRNA

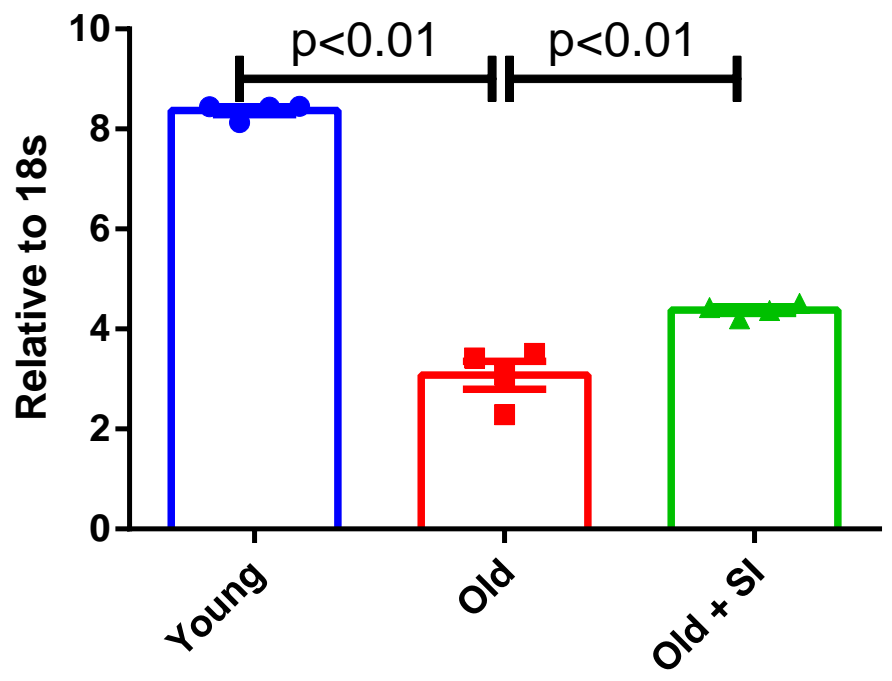

Atp5e mRNA

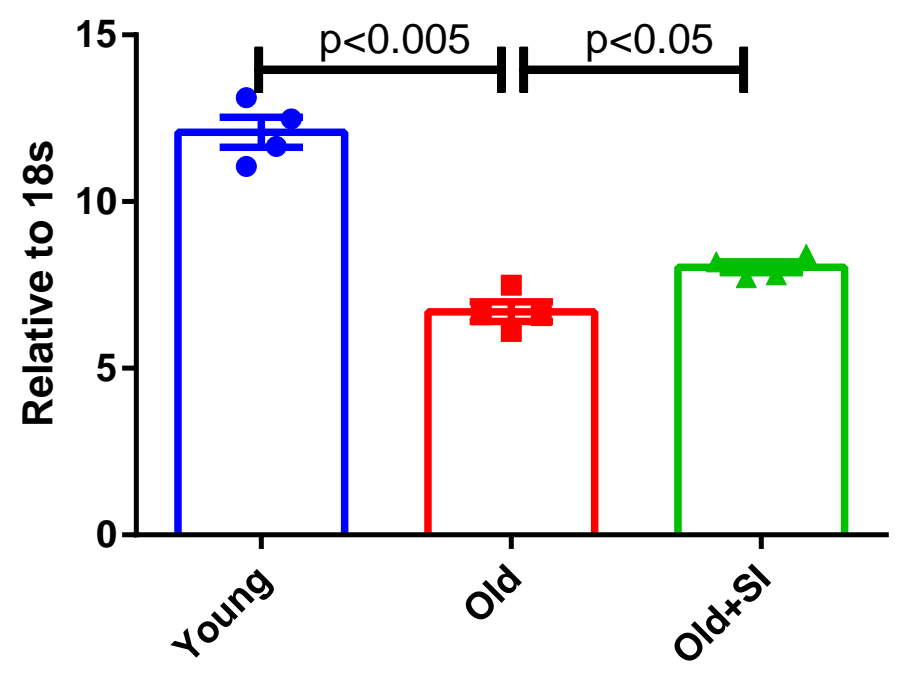

